\title{
Evaluation of the new UKCA climate-composition model - Part 2: The Troposphere
}

\author{
F. M. O'Connor ${ }^{1}$, C. E. Johnson ${ }^{1}$, O. Morgenstern ${ }^{2, *}$, N. L. Abraham ${ }^{2}$, P. Braesicke ${ }^{2}$, M. Dalvi ${ }^{1}$, G. A. Folberth ${ }^{1}$, \\ M. G. Sanderson ${ }^{1}$, P. J. Telford ${ }^{2}$, A. Voulgarakis ${ }^{3}$, P. J. Young ${ }^{2, * *}$, G. Zeng ${ }^{2, *}$, W. J. Collins ${ }^{1, * * *}$, and J. A. Pyle ${ }^{2}$ \\ ${ }^{1}$ Met Office Hadley Centre, Exeter, UK \\ ${ }^{2}$ NCAS-Climate-Chemistry, Cambridge University, UK \\ ${ }^{3}$ Department of Physics, Imperial College, UK \\ * now at: NIWA, Lauder, New Zealand \\ ** now at: Lancaster Environment Centre, Lancaster University, UK \\ *** now at: Department of Meteorology, Reading University, Reading, UK
}

Correspondence to: F. M. O’Connor (fiona.oconnor@metoffice.gov.uk)

Received: 1 February 2013 - Published in Geosci. Model Dev. Discuss.: 6 March 2013

Revised: 22 November 2013 - Accepted: 10 December 2013 - Published: 10 January 2014

\begin{abstract}
In this paper, we present a description of the tropospheric chemistry component of the UK Chemistry and Aerosols (UKCA) model which has been coupled to the Met Office Hadley Centre's HadGEM family of climate models. We assess the model's transport and scavenging processes, in particular focussing on convective transport, boundary layer mixing, wet scavenging and inter-hemispheric exchange. Simulations with UKCA of the short-lived radon tracer suggest that modelled distributions are comparable to those of other models and the comparison with observations indicate that apart from a few locations, boundary layer mixing and convective transport are effective in the model as a means of vertically redistributing surface emissions of radon. Comparisons of modelled lead tracer concentrations with observations suggest that UKCA captures surface concentrations in both hemispheres very well, although there is a tendency to underestimate the observed geographical and interannual variability in the Northern Hemisphere. In particular, UKCA replicates the shape and absolute concentrations of observed lead profiles, a key test in the evaluation of a model's wet scavenging scheme. The timescale for inter-hemispheric transport, calculated in the model using a simple krypton tracer experiment, does appear to be long relative to other models and could indicate deficiencies in tropical deep convection and/or insufficient boundary layer mixing. We also describe the main components of the tropospheric chemistry and evaluate it against observations
\end{abstract}

and other tropospheric chemistry models. In particular, from a climate forcing perspective, present-day observed surface methane concentrations and tropospheric ozone concentrations are reproduced very well by the model, thereby making it suitable for long centennial integrations as well as studies of biogeochemical feedbacks. Results from both historical and future simulations with UKCA tropospheric chemistry are presented. Future projections of tropospheric ozone vary with the Representative Concentration Pathway (RCP). In RCP2.6, for example, tropospheric ozone increases up to 2010 and then declines by $13 \%$ of its year-2000 global mean by the end of the century. In RCP8.5, tropospheric ozone continues to rise steadily throughout the 21 st century, with methane being the main driving factor. Finally, we highlight aspects of the UKCA model which are undergoing and/or have undergone recent developments and are suitable for inclusion in a next-generation Earth System Model.

\section{Introduction}

Atmospheric chemistry, aerosols, and the biosphere are important features of the Earth System (ES). The atmospheric abundance and lifetime of many greenhouse gases, such as methane $\left(\mathrm{CH}_{4}\right)$ and nitrous oxide $\left(\mathrm{N}_{2} \mathrm{O}\right)$, depend on atmospheric chemistry. Likewise, heterogeneous chemistry involving reactive chlorine from chlorofluorocarbons (CFCs) 
causes stratospheric ozone $\left(\mathrm{O}_{3}\right)$ depletion with implications for surface ultraviolet radiation (Madronich et al., 1998) and stratospheric dynamics (e.g. Butchart et al., 2003). Of significant importance in the troposphere are reactive gases such as carbon monoxide (CO), nitrogen oxides $\left(\mathrm{NO}_{\mathrm{x}}=\mathrm{NO}+\mathrm{NO}_{2}\right)$, and volatile organic compounds (VOCs) which do not have a direct radiative effect but still influence the climate indirectly by affecting the abundance of greenhouse gases such as $\mathrm{CH}_{4}$ and tropospheric $\mathrm{O}_{3}$. Atmospheric chemistry in the ES also influences climate through the formation of inorganic and organic aerosols (e.g. Seinfeld and Pandis, 2006). Together, tropospheric $\mathrm{O}_{3}, \mathrm{NO}_{\mathrm{x}}$, sulphur dioxide $\left(\mathrm{SO}_{2}\right)$, and aerosols contribute to poor air quality and have detrimental impacts on human health (e.g. WHO, 2003) and/or natural and managed ecosystems (e.g. Ashmore, 2005).

The role of feedbacks, such as chemistry-climate and chemistry-biosphere feedbacks, is also being recognised (e.g. Johnson et al., 2001; Sitch et al., 2007), and ES models (ESMs) increasingly include aspects of atmospheric chemistry. For example, climate change influences the timescale of stratospheric $\mathrm{O}_{3}$ recovery (e.g. Waugh et al., 2009) and may affect future surface $\mathrm{O}_{3}$ concentrations through changes in vegetation and biogenic VOC emissions (e.g. Sanderson et al., 2003). As a result, there is a need to implement stratospheric, tropospheric, and troposphere-stratosphere chemistry and aerosol schemes in climate models to understand and quantify interactions between climate, atmospheric composition, and other earth-system components such as vegetation and hydrology.

To address this increasing need, the objective of the UK Chemistry and Aerosols (UKCA) project is to develop a state-of-the-art chemistry and aerosol model, capable of simulating atmospheric composition from the troposphere to the upper stratosphere. The various configurations of UKCA are described and evaluated in a series of papers. Part 1 of this series, by Morgenstern et al. (2009), provided a detailed description and evaluation of the stratospheric configuration of UKCA. It was used to explore the impact of the Montreal Protocol on climate by Morgenstern et al. (2008). Furthermore, the stratospheric configuration participated in the recent Stratospheric Processes and their Role in Climate (SPARC) Report on the Evaluation of ChemistryClimate Models (Eyring et al., 2010a) and was used in the World Meteorological Organization (WMO) ozone assessment (WMO, 2011). Part 3 of the series, by Mann et al. (2014), will focus on the aerosol component of UKCA, called GLOMAP-mode. Part 4 will discuss an extension to the tropospheric chemistry scheme with enhanced coupling between the atmosphere and biosphere (Folberth et al., 2014). This paper is Part 2 of the series and will focus on the gas-phase tropospheric chemistry configuration of UKCA in a chemistry-climate model. The climate model has already been used by O'Connor et al. (2009) to evaluate the interactions between climate model biases and tropospheric chemistry. Telford et al. (2010) used this configuration to explore the impact of climate-induced changes by the Pinatubo volcanic eruption on biogenic emissions and atmospheric composition. More recently, Archibald et al. (2011) explored the impacts of odd hydrogen regeneration and recycling in the oxidation of isoprene for past, present, and future atmospheres using this configuration. It has also been used in all centennial simulations of the Hadley Centre's ESM, HadGEM2-ES (Collins et al., 2011), for the fifth Coupled Model Intercomparison Project (CMIP5) and in the recent Atmospheric Chemistry and Climate Model Intercomparison Project (ACCMIP; Lamarque et al., 2013). In this paper, we provide a more detailed description and evaluation of the tropospheric chemistry configuration of the UKCA model. In Sect. 2, we will introduce the climate models to which UKCA has been coupled. We will then assess aspects of the tracer transport which form the basis of UKCA in Sect. 3. In Sect. 4, we will describe the two tropospheric chemistry schemes in this configuration of UKCA and in Sect. 5, we will compare UKCA to both observations and other chemistry models. A number of sensitivity experiments were carried out to explore the causes of certain biases and/or assess the impact of the differences between the two chemistry schemes described; these experiments are discussed in Sect. 6. Results from the transient CMIP5 integrations using the HadGEM2-ES ESM, which included the tropospheric chemistry configuration of UKCA, are presented in Sect. 7. Finally, in Sect. 8, we discuss the results, draw some conclusions about UKCA's current performance, and provide a summary of ongoing developments which will be included in future versions of the UKCA model.

\section{The climate models, HadGEM1 and HadGEM2}

The UK Chemistry and Aerosol (UKCA) model has been successfully coupled to a number of Met Office Hadley Centre climate models, HadGEM1 (Johns et al., 2006; Martin et al., 2006) and HadGEM2 (The HadGEM2 Development Team: Martin et al., 2011; Collins et al., 2011), all of which are based on the UK Met Office's Unified Model (MetUM). The model uses a hybrid height vertical co-ordinate, and in the L38 configuration, a typical model layer thickness at the tropopause is of the order of 800-1000 m, with the model lid at approximately $39 \mathrm{~km}$ (Fig. 2b in The HadGEM2 Development Team: Martin et al., 2011). In the L60 configuration (Hardiman et al., 2010), the model levels below $16 \mathrm{~km}$ are identical to those in the L38 configuration, whereas above $16 \mathrm{~km}$, L60 has 32 levels in comparison with 10 levels in the L38 configuration. The dynamical timestep for the two vertical configurations is 30 and $20 \mathrm{~min}$, respectively. The horizontal resolution is typically N96, i.e. $1.875^{\circ} \times 1.25^{\circ}$, which equates to about $140 \mathrm{~km}$ at mid-latitudes. For a detailed description and evaluation of the N96L38 configuration of HadGEM2, and how it differs from HadGEM1, the 
reader is referred to the paper by The HadGEM2 Development Team: Martin et al. (2011). UKCA has also successfully been coupled to the HadGEM3 model (Hewitt et al., 2011).

The configuration used here is an atmosphere-only version of HadGEM2 with sea surface temperature and sea ice data sets taken from the second Atmosphere Model Intercomparison Project (www-pcmdi.llnl.gov/projects/amip); the resolution was N96L38. Hereinafter, it is referred to as the HadGEM2-UKCA coupled model or simply HadGEM2UKCA.

\section{Tracer transport and physical processing}

The coupling between the UKCA chemistry-aerosol model and the HadGEM climate models is based on MetUM. UKCA uses components of the Unified Model for the largescale advection, convective transport, and boundary layer mixing of its chemical and aerosol tracers. The large-scale transport is based on the dynamical core implemented in MetUM by Davies et al. (2005) with semi-Lagrangian advection and conservative and monotone treatment of tracers (Priestley, 1993). Tracers in the boundary layer are mixed using the boundary layer scheme from Lock et al. (2000) which includes a representation of non-local mixing in unstable layers and an explicit entrainment parameterization. Convective transport is treated according to the mass-flux scheme of Gregory and Rowntree (1990) with extensions described in Martin et al. (2006).

In the development phase of chemistry-climate models and chemistry transport models, simple tracer experiments (e.g. Rind and Lerner, 1996; Jacob et al., 1997; Rasch et al., 2000) are useful as a means of assessing some of the dynamical processes of the base models. Here, we perform a number of experiments to evaluate the convective transport, wet scavenging, inter-hemispheric transport, and boundary layer mixing in the HadGEM2-UKCA coupled model. Furthermore, an inert tracer is used to evaluate the degree to which global tracer mass is conserved. Before we describe and evaluate the tracer transport experiments, a brief description of the wet and dry deposition schemes that are employed in the model are outlined in the following sections.

\subsection{Dry deposition}

The process by which chemical species are transferred to the surface and removed from the atmosphere in the absence of precipitation is represented in the tropospheric chemistry configuration of UKCA by one of two dry deposition schemes. The first scheme uses prescribed deposition velocities at $1 \mathrm{~m}$ above the ground (Ganzeveld and Lelieveld, 1995; Sander and Crutzen, 1996; Giannakopoulos, 1998, and references therein), which are then extrapolated to the mid-point of the lowest model level according to Sorteberg and Hov (1996). This scheme is used in the tracer experiment assessing wet scavenging in Sect. 3.4 and matches what was evaluated in the TOMCAT chemical transport model (CTM) by Giannakopoulos (1998) and Giannakopoulos et al. (1999).

The preferred dry deposition scheme in UKCA is analogous to an electrical circuit, in which the transport of material to the surface is governed by three resistances, $r_{\mathrm{a}}, r_{\mathrm{b}}$, and $r_{\mathrm{c}}$ in series and the deposition velocity $V_{\mathrm{d}}\left(\mathrm{m} \mathrm{s}^{-1}\right)$ is related to the resistances as follows (Wesely, 1989):

$V_{\mathrm{d}}=\frac{1}{r_{\mathrm{t}}}=\frac{1}{r_{\mathrm{a}}+r_{\mathrm{b}}+r_{\mathrm{c}}}$.

A full description of this representation of dry deposition is given by Seinfeld and Pandis (2006) and Smith et al. (2000) and is only briefly described here. The first resistance, $r_{\mathrm{a}}$, the so-called aerodynamic resistance, represents the resistance to transport of material through the boundary layer to a thin layer of air just above the surface. It is calculated from the wind profile, taking into account atmospheric stability and the surface roughness as follows:

$r_{\mathrm{a}}=\frac{\ln \left(z / z_{0}\right)-\Psi}{k \times u^{*}}$,

where $z_{0}$ is the roughness length, $\Psi$ is the Businger dimensionless stability function, $k$ is von Karman's constant, and $u^{*}$ is the friction velocity.

The second resistance term, $r_{\mathrm{b}}$, called the quasi-laminar resistance, refers to the resistance to transport through the thin layer of air close to the surface. Finally, the third resistance, $r_{\mathrm{c}}$, is called the surface or canopy resistance and represents the resistance to uptake at the surface itself. It is dependent on the chemical and physical properties of the species as well as the absorbing surface. Under ideal conditions, $r_{\mathrm{c}}$ can be related to surface conditions, time of day, and season. It is often represented by further application of the electrical resistance analogy and is called the "Big-leaf" model (Smith et al., 2000; Seinfeld and Pandis, 2006). This is illustrated schematically in Fig. 1. There are 9 surface types considered in the model; these are broad-leaved trees, needle-leaf trees, C3 and C4 grass, shrub, urban, water, bare soil, and land ice. In the case of simulations with non-interactive vegetation (as is the case here), these are prescribed using distributions from the IGBP (International Geosphere-Biosphere Programme). The multiple resistances are calculated for the 9 surface types within a grid box and are then combined to give a grid-box mean deposition velocity and a first-order loss rate. However, not all the terms will be non-zero at a particular grid box and a particular time. In vegetated areas, for example, plant stomata open during daylight hours and deposition to the interior of the plant via the stomata can occur. This acts as a major daytime sink for $\mathrm{O}_{3}$, for example. At night, the stomata are closed and the major loss is to the cuticles and underlying soil. An important difference between 
the two dry deposition schemes is that the dry deposition loss rates are only applied in the bottom model level with the first scheme but applied in all model levels within the boundary layer with the second scheme. This second scheme has been implemented in UKCA in a similar way to its implementation in the STOCHEM model by Sanderson et al. (2006) and Sanderson et al. (2007) and is the scheme of choice. Therefore, the HadGEM2-UKCA coupled model integrations with chemistry being evaluated here use the multiple resistance approach to dry deposition, whereas the simple tracer experiments use prescribed deposition velocities.

\subsection{Wet deposition}

The wet deposition scheme in UKCA for the tropospheric gas-phase species is the same scheme as was implemented and validated in the TOMCAT CTM by Giannakopoulos (1998) and Giannakopoulos et al. (1999). It is parameterized as a first-order loss rate, calculated as a function of the climate model's three-dimensional convective and large-scale precipitation. It follows a scheme originally developed by Walton et al. (1988). At each grid box, the scavenging rate $r$ can be written as follows:

$r=S_{j} \times p_{j}(l)$,

where $S_{j}$ is the scavenging coefficient for precipitation type $j$ $\left(\mathrm{cm}^{-1}\right)$ and $p_{j}(l)$ is the precipitation rate for type $j$ (convective or large-scale) from model level $l\left(\mathrm{~cm} \mathrm{~h}^{-1}\right)$. The scheme uses the scavenging coefficient values for nitric acid $\left(\mathrm{HNO}_{3}\right)$ proposed by Penner et al. (1991), i.e. $2.4 \mathrm{~cm}^{-1}$ and $4.7 \mathrm{~cm}^{-1}$ for large-scale and convective precipitation, respectively, but which are scaled down for the individual species by the fraction of each species in the aqueous phase. The fraction in the aqueous phase, $f_{\text {aq }}$, from Henry's Law is as follows:

$f_{\text {aq }}=\frac{L \times H_{\text {eff }} \times R \times T}{1+L \times H_{\text {eff }} \times R \times T}$,

where $L$ is the liquid water content, $R$ is the gas constant, $T$ is temperature, and $H_{\text {eff }}$ is the effective Henry's coefficient. $H_{\text {eff }}$ not only depends on the solubility of a species, but also includes the effects of dissociation and complex formation. Tables 1 and 2 show the parameters required to calculate the fraction of each soluble species in the aqueous phase for the two tropospheric chemistry schemes that will be described here.

It is also assumed that precipitation type $j$ only occurs over a fraction of the grid box. This fraction is taken to be 1.0 and 0.3 for large-scale and convective precipitation, respectively, and is taken into account when calculating a grid-box mean scavenging rate for both types of precipitation which are then added together. The scheme is evaluated fully using a lead$210\left({ }^{210} \mathrm{~Pb}\right)$ tracer experiment in Sect. 3.4.

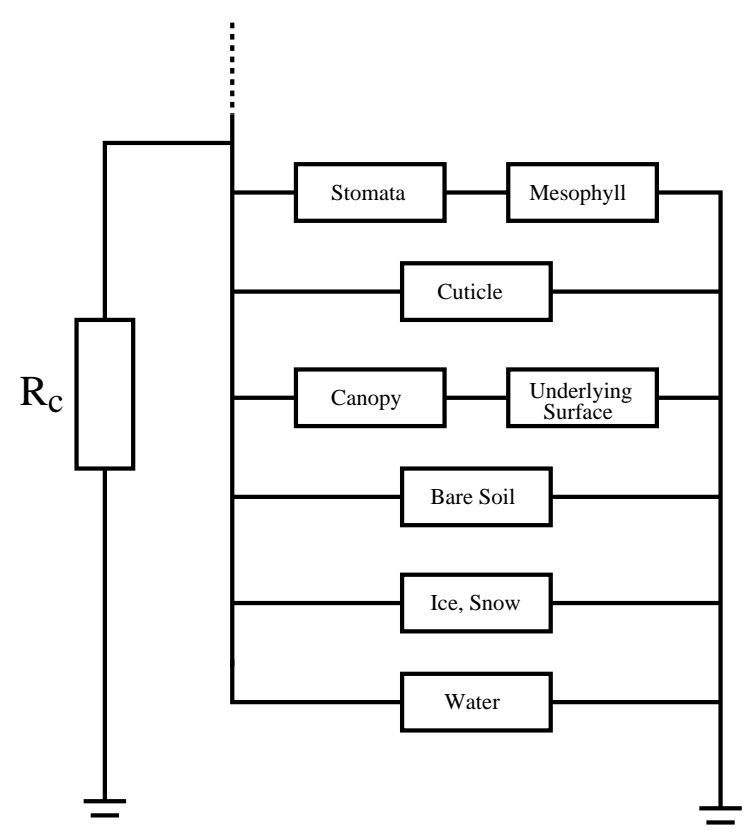

Fig. 1. Schematic of the terms used to calculate the overall surface or canopy resistance term, $r_{\mathrm{c}}$, for dry deposition. Adapted from Sanderson et al. (2007).

\subsection{Convective transport and boundary layer mixing}

We assess the dynamical performance of HadGEM2 by separating the dynamics of the model from the chemistry by using a fixed-lifetime species. The short-lived radioisotope radon$222\left({ }^{222} \mathrm{Rn}\right)$ is such a tracer and simulations of ${ }^{222} \mathrm{Rn}$ have been extensively used to evaluate convective and synopticscale processes in global models (e.g. Rind and Lerner, 1996; Jacob et al., 1997; Stevenson et al., 1998; Collins et al., 2002; Hauglustaine et al., 2004) and to a lesser extent in regional models (e.g. Lin et al., 1996; Chevillard et al., 2002). It is emitted from non-frozen soils due to radioactive decay of radium-226 $\left({ }^{226} \mathrm{Ra}\right)$. Although emissions of ${ }^{222} \mathrm{Rn}$ depend on soil and meteorological conditions, a very simple emission scenario is used here. Surface ${ }^{222} \mathrm{Rn}$ emissions are prescribed as 1.0 atom $\mathrm{cm}^{-2} \mathrm{~s}^{-1}$ over all land between $60^{\circ} \mathrm{S}$ and $60^{\circ} \mathrm{N}$ except Greenland and emitted into the lowest model layer. This prescribed emission rate matches the best mean estimate of 1.2 atom $\mathrm{cm}^{-2} \mathrm{~s}^{-1}$ from a compilation of radon flux measurements by Turekian et al. (1977). Emissions between $60^{\circ}$ and $70^{\circ} \mathrm{N}$ are specified as 0.5 atom cm $\mathrm{cm}^{-2} \mathrm{~s}^{-1}$ (to account for soil freezing) with no emissions poleward of $60^{\circ} \mathrm{S}$ and $70^{\circ} \mathrm{N}$. Emissions from oceanic regions are approximately two orders of magnitude lower than land emissions (Broecker et al., 1967) and are neglected here, giving a global annual total emission of $15 \mathrm{~kg} \mathrm{yr}^{-1}$. These emissions match those used in the intercomparison study of Rasch et al. (2000). Due to its lack of solubility in water, loss of ${ }^{222} \mathrm{Rn}$ from the atmosphere is solely by radioactive decay (through a number of shortlived daughters) to ${ }^{210} \mathrm{~Pb}$ with a half-life of 3.8 days. 
Table 1. Values required to calculate the effective Henry's Law coefficient for the soluble species included in the UKCA standard tropospheric (StdTrop) scheme, where $\mathrm{Me}=\mathrm{CH}_{3}, \mathrm{Et}=\mathrm{C}_{2} \mathrm{H}_{5}$, and $\mathrm{Pr}=\mathrm{C}_{3} \mathrm{H}_{7}$. References: (1) Jacob (2000); (2) Regimbal and Mozurkewich (1997); (3) CRC (2003); (4) Lelieveld and Crutzen (1991); (5) Hanson et al. (1992); (6) O’Sullivan et al. (1996); (7) Staudinger and Roberts (2001); and (8) Becker et al. (1996).

\begin{tabular}{lrrrrr}
\hline \multirow{2}{*}{ Species } & \multicolumn{2}{c}{ Henry's Law Data } & \multicolumn{2}{c}{ Dissociation Data } & \\
& $K_{\mathrm{H}}(298 \mathrm{~K})$ & $-\Delta H / R$ & $K_{\mathrm{a}}(298 \mathrm{~K})$ & $-\Delta H / R$ & Reference \\
& $\mathrm{M} \mathrm{atm}{ }^{-1}$ & $\mathrm{~K}^{-1}$ & $\mathrm{M}$ & $\mathrm{K}^{-1}$ & \\
\hline $\mathrm{NO}_{3}$ & $2.0 \mathrm{E}+00$ & 2000.0 & $0.0 \mathrm{E}+00$ & 0.0 & 1 \\
$\mathrm{~N}_{2} \mathrm{O}_{5}$ & $2.1 \mathrm{E}+05$ & 8700.0 & $2.0 \mathrm{E}+01$ & 0.0 & As for $\mathrm{HONO}_{2}$ \\
$\mathrm{HO}_{2} \mathrm{NO}_{2}$ & $1.3 \mathrm{E}+04$ & 6900.0 & $1.0 \mathrm{E}-05$ & 0.0 & $2 ; 3$ \\
$\mathrm{HONO}_{2}$ & $2.1 \mathrm{E}+05$ & 8700.0 & $2.0 \mathrm{E}+01$ & 0.0 & 4 \\
$\mathrm{HO}_{2}$ & $4.0 \mathrm{E}+03$ & 5900.0 & $2.0 \mathrm{E}-05$ & 0.0 & $5 ; 1$ \\
$\mathrm{H}_{2} \mathrm{O}_{2}$ & $8.3 \mathrm{E}+04$ & 7400.0 & $2.4 \mathrm{E}-12$ & -3730.0 & 6 \\
$\mathrm{HCHO}^{\mathrm{MeOO}}$ & $3.3 \mathrm{E}+03$ & 6500.0 & $0.0 \mathrm{E}+00$ & 0.0 & 7 \\
$\mathrm{MeOOH}$ & $2.0 \mathrm{E}+03$ & 6600.0 & $0.0 \mathrm{E}+00$ & 0.0 & 7 \\
$\mathrm{HONO}$ & $3.1 \mathrm{E}+02$ & 5000.0 & $0.0 \mathrm{E}+00$ & 0.0 & 7 \\
$\mathrm{EtOOH}$ & $5.0 \mathrm{E}+01$ & 4900.0 & $5.6 \mathrm{E}-04$ & -1260.0 & 0.0 \\
$n-\mathrm{PrOOH}$ & $3.4 \mathrm{E}+02$ & 5700.0 & $0.0 \mathrm{E}+00$ & 0.0 & As for EtOOH \\
$i$-PrOOH & $3.4 \mathrm{E}+02$ & 5700.0 & $0.0 \mathrm{E}+00$ & 0.0 & As for EtOOH \\
$\mathrm{MeCOCH}$ & $3.4 \mathrm{E}+02$ & 5700.0 & $0.0 \mathrm{E}+00$ & 0.0 & As for EtOOH \\
\hline
\end{tabular}

Table 2. Values required to calculate the effective Henry's Law coefficient for the additional soluble species included in the UKCA standard tropospheric scheme with isoprene oxidation (TropIsop), where $\mathrm{Me}=\mathrm{CH}_{3}, \mathrm{IS}=\mathrm{C}_{5} \mathrm{H}_{7}$, ISON $=$ lumped peroxy alkyl nitrates (from $\mathrm{C}_{5} \mathrm{H}_{8}+\mathrm{NO}_{3}$ ) and hydroxy alkyl nitrates (from $\mathrm{ISO}_{2}+\mathrm{NO}$ ), MACR = lumped species consisting of methacrolein, methyl vinyl ketone and other $\mathrm{C}_{4}$ carbonyls from isoprene oxidation, HACET $=$ hydroxyacetone and other $\mathrm{C}_{3}$ ketones, MGLY= methylglyoxal and other $\mathrm{C}_{3}$ carbonyls, NALD = nitrooxy acetaldehyde, and Prpe $=\mathrm{C}_{3} \mathrm{H}_{5}$. References: (1) Staudinger and Roberts (2001); and (2) CRC (2003).

\begin{tabular}{lrrrrr}
\hline \multirow{2}{*}{ Species } & \multicolumn{2}{c}{ Henry's Law Data } & \multicolumn{2}{c}{ Dissociation Data } & \\
& $K_{\mathrm{H}}(298 \mathrm{~K})$ & $-\Delta H / R$ & $K 0_{\mathrm{H}}(298 \mathrm{~K})$ & $-\Delta H / R$ & Reference \\
& $\mathrm{M} \mathrm{atm}^{-1}$ & $\mathrm{~K}^{-1}$ & $\mathrm{M}$ & $\mathrm{K}^{-1}$ & \\
\hline ISOOH & $1.7 \mathrm{E}+06$ & 9700.0 & $0.0 \mathrm{E}+00$ & 0.0 & As for PrpeOOH from 1 \\
ISON & $3.0 \mathrm{E}+03$ & 7400.0 & $0.0 \mathrm{E}+00$ & 0.0 & 1 \\
MACROOH & $1.7 \mathrm{E}+06$ & 9700.0 & $0.0 \mathrm{E}+00$ & 0.0 & As for PrpeOOH from 1 \\
$\mathrm{HACET}$ & $1.4 \mathrm{E}+02$ & 7200.0 & $0.0 \mathrm{E}+00$ & 0.0 & 1 \\
$\mathrm{MGLY}$ & $3.5 \mathrm{E}+03$ & 7200.0 & $0.0 \mathrm{E}+00$ & 0.0 & 1 \\
$\mathrm{HCOOH}$ & $6.9 \mathrm{E}+03$ & 5600.0 & $1.8 \mathrm{E}-04$ & -1510.0 & $1 ; 2$ \\
$\mathrm{MeCO}_{3} \mathrm{H}$ & $7.5 \mathrm{E}+02$ & 5300.0 & $6.3 \mathrm{E}-09$ & 0.0 & 1 \\
$\mathrm{MeCO}_{2} \mathrm{H}$ & $4.7 \mathrm{E}+03$ & 6000.0 & $1.8 \mathrm{E}-05$ & 0.0 & $1 ; 2$ \\
\hline
\end{tabular}

Two additional tracers were included in the HadGEM2UKCA coupled simulation. Both represent the daughter product of ${ }^{222} \mathrm{Rn}$ and ${ }^{210} \mathrm{~Pb}$. The first ${ }^{210} \mathrm{~Pb}$ tracer accumulates in the atmosphere to test mass conservation (Sect. 3.5). The second ${ }^{210} \mathrm{~Pb}$ tracer is removed from the atmosphere through dry deposition (Sect. 3.1) and wet scavenging (Sect. 3.4) processes. All the tracers were initialised to zero, allowed to spin up for 4 months, and the simulation was then run for $5 \mathrm{yr}$. An additional experiment was also run for $5 \mathrm{yr}$, in which the mixing of ${ }^{222} \mathrm{Rn}$ by the boundary layer scheme was switched off, thereby enabling the relative importance of boundary layer mixing and convective transport to be assessed.
For ease of comparison with other models, units of $10^{-21} \mathrm{~mol} \mathrm{~mol}^{-1}$, equivalent to $1.52 \mathrm{pCi} \mathrm{m}^{-3}$ at standard temperature and pressure (STP), are used. Figure 2 shows the multi-annual zonal mean distribution of ${ }^{222} \mathrm{Rn}$ from the two experiments for the June-July-August (JJA) period. It shows that for the control experiment, the zonal mean distribution from HadGEM2-UKCA is similar to the models that participated in the World Climate Research Program (WCRP) intercomparison (Jacob et al., 1997) and other models (e.g. Dentener et al., 1999; Hauglustaine et al., 2004). Surface concentrations show a maximum in the northern mid-latitudes of $20-30 \times 10^{-21} \mathrm{~mol} \mathrm{~mol}^{-1}$, in close agreement with Jacob et al. (1997). From the HadGEM2-UKCA distribution, 

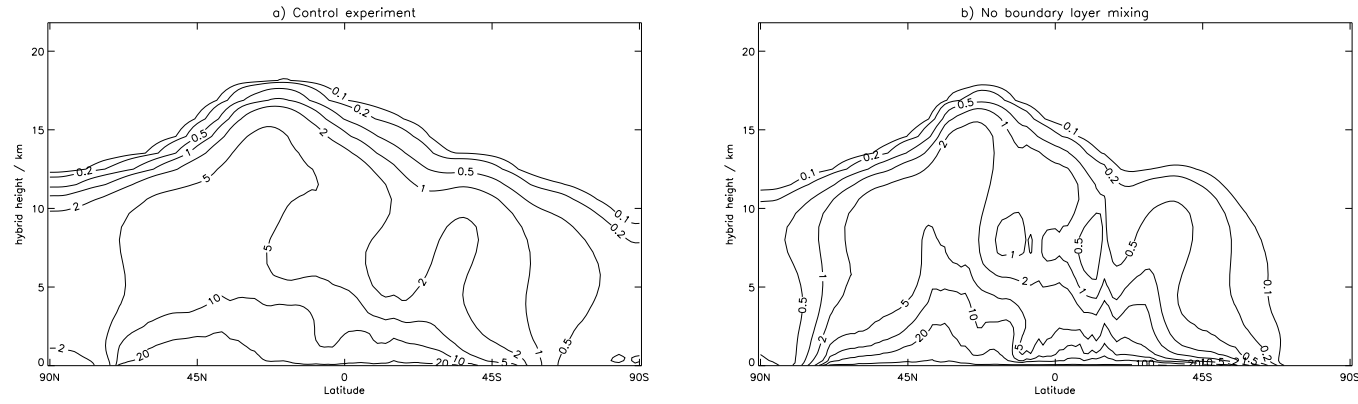

Fig. 2. Multi-annual zonal mean radon concentrations in units of $10^{-21} \mathrm{~mol} \mathrm{~mol}^{-1}$ for (a) the control experiment and (b) an experiment with no boundary layer mixing for the June-July-August (JJA) time period.

there is evidence of deep convection in the tropics, midlevel convection at northern mid-latitudes, and meridional gradients in the lower and mid troposphere. In the model intercomparison of Jacob et al. (1997), the GISS model showed a secondary maximum in the tropical upper troposphere due to frequent deep convection; a similar feature is evident in HadGEM2-UKCA albeit less pronounced. When boundary layer mixing was switched off (Fig. 2b), concentrations at the surface exceed $50 \times 10^{-21} \mathrm{~mol} \mathrm{~mol}^{-1}$ and tropical upper tropospheric concentrations only reach a maximum of $2 \times 10^{-21} \mathrm{~mol} \mathrm{~mol}^{-1}$. In the study by Stockwell et al. (1998), they found that if the planetary boundary layer was not well mixed, this affected the amount of ${ }^{222} \mathrm{Rn}$ which could be lifted aloft in convective updrafts. This experiment with HadGEM2-UKCA clearly indicates the role of boundary layer mixing in determining the vertical redistribution of ${ }^{222} \mathrm{Rn}$ from its source regions.

Figure 3 shows a comparison of climatological surface ${ }^{222} \mathrm{Rn}$ observations with HadGEM2-UKCA modelled concentrations at a number of different surface sites, spanning the latitude range $53^{\circ} \mathrm{N}$ to $67^{\circ} \mathrm{S}$, and including coastal (e.g. Mace Head), continental (e.g. Socorro), and remote marine (e.g. Amsterdam Island) sites. There are some stations where the model performs well (e.g. Amsterdam Island, Bermuda). At the remote site of Amsterdam Island, local emissions do not influence the observation site and these measurements can be considered truly representative of long-range transport from Africa (Dentener et al., 1999). In this case, HadGEM2-UKCA matches the observed concentrations quite well. Bermuda, on the other hand, is a surface site where concentrations are sensitive to planetary boundary layer ventilation (Allen et al., 1996). The observed seasonality and the absolute monthly mean concentrations are captured by HadGEM2-UKCA particularly well. At the continental site of Socorro, there is an observed autumn to winter increase associated with seasonality in emissions and/or mixing by Allen et al. (1996). HadGEM2-UKCA represents the wintertime observations quite well. However, it fails to capture the correct seasonal cycle or the observed autumn to winter increase, but no seasonality in emissions was taken into account. At Bombay, HadGEM2-UKCA significantly overestimates surface concentrations by a factor of 2 during the northern hemisphere winter, suggesting that there is insufficient ventilation out of the boundary layer in that region. HadGEM2-UKCA also does not capture the correct seasonal cycle at Dumont d'Urville, although it manages to simulate concentrations comparable to the observed concentrations during JJA. Other models also do poorly at this location (e.g. Hauglustaine et al., 2004) and Josse et al. (2004) have attributed this in the MOCAGE model to the lack of penetration of polar storms onto the continent due to sea ice extent. Some studies have also indicated that the use of a northwards-decreasing emission source between 30 and $70^{\circ} \mathrm{N}$ could improve simulations in the Northern Hemisphere (Conen and Robertson, 2002; Gupta et al., 2004), particularly north of $50^{\circ} \mathrm{N}$ (Robertson et al., 2005). This may well improve the simulations from HadGEM2UKCA at Chester and Socorro but is likely to make comparisons at Mace Head worse. Overall, the performance of the HadGEM2-UKCA simulations suggest that there may be insufficient boundary layer ventilation at some locations in the Northern Hemisphere.

A comparison of modelled and measured vertical profiles of ${ }^{222} \mathrm{Rn}$ at two different locations can be seen in Fig. 4. The observations from Moffett Field, California $\left(37.4^{\circ} \mathrm{N}, 122.0^{\circ} \mathrm{W}\right)$ were taken onboard 11 flights in the June-August 1994 time period (Kritz et al., 1998). Of the 11 flights, only 1 took place during July and the modelled profiles were taken from June and August only. HadGEM2UKCA overestimates ${ }^{222} \mathrm{Rn}$ concentrations at the surface over Moffett Field; this may be attributed to the limited horizontal resolution of the model when comparing with measurements taken at a coastal site where strong horizontal gradients exist (Stockwell et al., 1998). Above the boundary layer, the coupled HadGEM2-UKCA model is in very close agreement with the observed concentrations between 2 and $10 \mathrm{~km}$. However, it fails to capture the enhanced concentrations at $11-13 \mathrm{~km}$, associated with long-range transport of Asian air following deep convection (Stockwell et al., 1998). This discrepancy has been found in other models (e.g. 

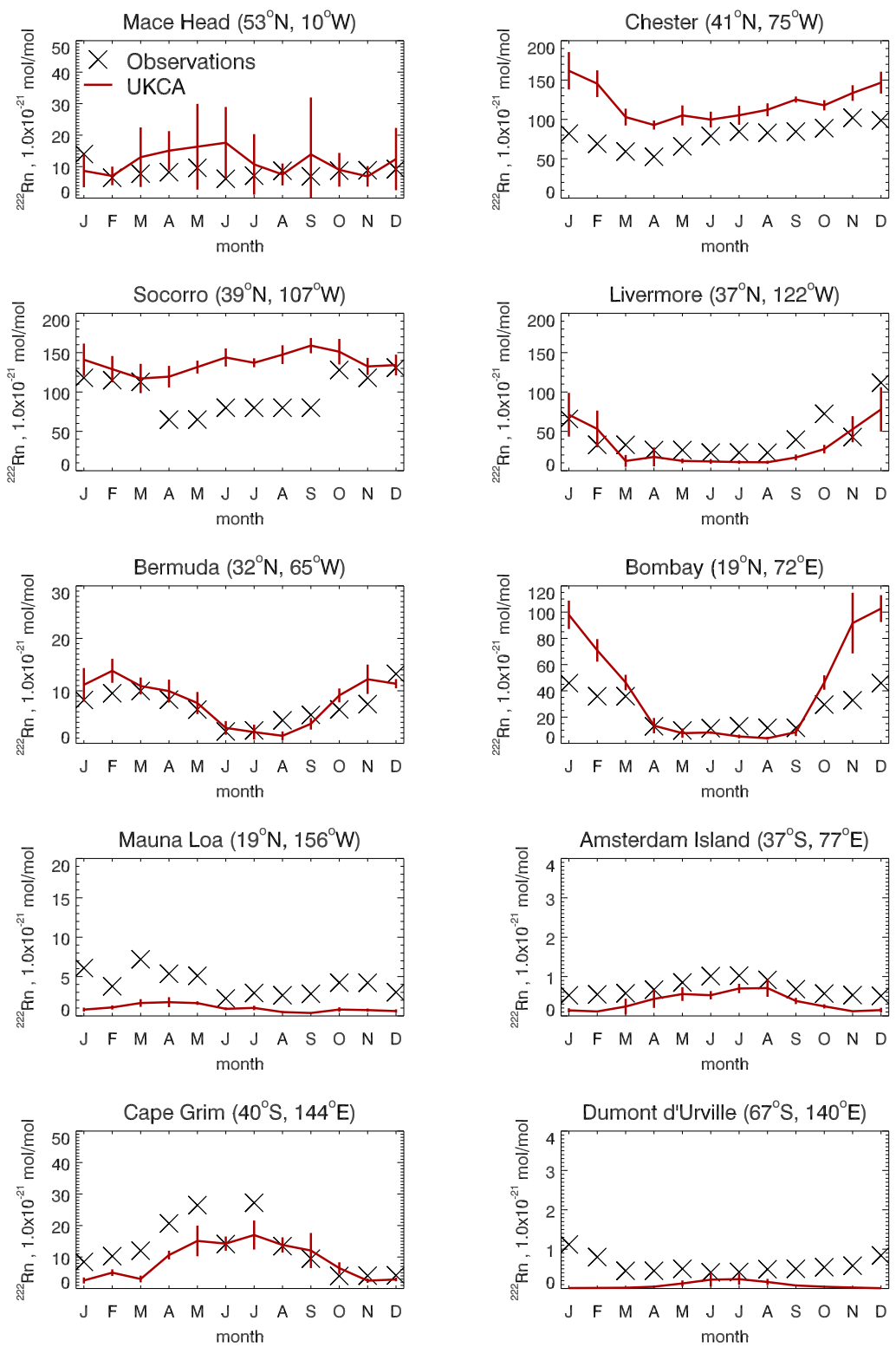

Fig. 3. Comparison of modelled multi-annual monthly mean surface radon concentrations in units of $10^{-21} \mathrm{~mol} \mathrm{~mol}^{-1}$ with surface observations for a number of sites spanning the latitude range $53^{\circ} \mathrm{N}-67^{\circ} \mathrm{S}$. Observations are shown as black crosses and the UKCA model is shown in red. The red vertical lines represent \pm 1 standard deviation about the modelled multi-annual monthly mean values. Measurements are taken from Hauglustaine et al. (2004) and references therein.

Stockwell et al., 1998; Gupta et al., 2004) and could be related to a lack of deep convection over Asia, insufficient long-range transport from Asia, and/or an underestimate in the Asian emissions in the simple emissions scenario used here (Jacob et al., 1997; Dentener et al., 1999).

The Nova Scotia measurements were taken during 9 North Atlantic Regional Experiment (NARE) flights over the North Atlantic Ocean and close to Nova Scotia and New Brunswick, Canada, between 16 and 31 August 1993 (Zaucker et al., 1996). For comparison purposes, the modelled profiles are multi-annual means for August only. The coupled HadGEM2-UKCA model replicates the observed shape of the vertical profile and the magnitude of the observed concentrations in the $2-5 \mathrm{~km}$ region very well. It underestimates concentrations in the lowest $1 \mathrm{~km}$, although the observations show a great deal of variability there. Enhanced concentrations were observed at 5-6 km, but HadGEM2-UKCA indicates a minimum at $6 \mathrm{~km}$ and places the enhancement aloft. However, on the whole, the 

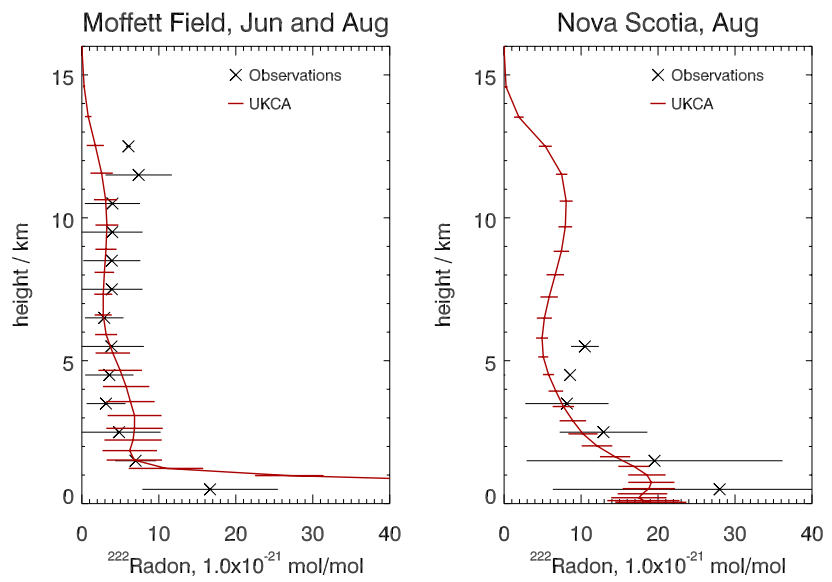

Fig. 4. Comparison of modelled profiles of radon in units of $10^{-21} \mathrm{~mol} \mathrm{~mol}^{-1}$ with observations at two different sites. Observations are shown as black crosses and the UKCA model is shown in red. Measurements from Moffett Field and Nova Scotia are taken from Kritz et al. (1998) and Zaucker et al. (1996), respectively.

performance of the HadGEM2-UKCA coupled model in relation to the vertical profiles of ${ }^{222} \mathrm{Rn}$ is very good.

Further evaluation of tropical deep convection in MetUM in comparison with observations and other models has been carried out by Russo et al. (2011) and Hoyle et al. (2011).

\subsection{Wet scavenging}

As was mentioned in Sect. 3.3, the ${ }^{222} \mathrm{Rn}$ experiment above included the daughter product, ${ }^{210} \mathrm{~Pb}$, which was subject to wet and dry deposition. Dry deposition velocities at $1 \mathrm{~m}$ were prescribed as follows: $0.2 \mathrm{~cm} \mathrm{~s}^{-1}$ over land and $0.05 \mathrm{~cm} \mathrm{~s}^{-1}$ over the ocean (Balkanski et al., 1993) and extrapolated to the mid-point of the lowest model level according to Sorteberg and Hov (1996) assuming neutral stability conditions. For wet deposition, ${ }^{210} \mathrm{~Pb}$ is assumed to attach itself to soluble sub-micron aerosols and scavenging was treated as a firstorder loss process as described in Sect. 3.2. For the scavenging coefficients, we used values of $2.4 \mathrm{~cm}^{-1}$ for dynamic precipitation and $4.7 \mathrm{~cm}^{-1}$ for convective precipitation, which are appropriate for highly soluble gaseous species such as $\mathrm{HNO}_{3}$ (Penner et al., 1991). Radioactive decay of ${ }^{210} \mathrm{~Pb}$ (half-life of $22.3 \mathrm{yr}$ ) was not taken into account.

In order to assess the modelled surface ${ }^{210} \mathrm{~Pb}$ concentrations, climatological observations were obtained at 20 global sites spanning latitudes $90^{\circ} \mathrm{S}-77^{\circ} \mathrm{N}$ from the Surface Air Sampling Program database of the Environmental Measurements Laboratory (EML) (http://esd.lbl.gov/research/ facilities/eml/). Figures 5 and 6 show comparisons of multiannual surface ${ }^{210} \mathrm{~Pb}$ concentrations from HadGEM2-UKCA with climatological observed surface ${ }^{210} \mathrm{~Pb}$ concentrations in the Northern and Southern hemispheres, respectively. They show that the HadGEM2-UKCA coupled model is in excellent agreement with the measurements in terms of the seasonal cycle and absolute concentrations at some sites (e.g. Moosonee, Beaverton, Chester, and Cape Grim). At Barrow, for example, HadGEM2-UKCA correctly simulates the wintertime maximum associated with long-range boundary layer transport of Eurasian air which gives rise to the Arctic haze phenomenon (Barrie, 1986). At Kap Tobin, HadGEM2UKCA does not simulate the seasonal cycle indicated by the observations. However, the measurement record at this site is quite short (approximately $2 \mathrm{yr}$ ) relative to the other surface sites. The model also tends to underestimate surface ${ }^{210} \mathrm{~Pb}$ concentrations and fails to capture the seasonal cycle at the South Pole; this was also evident in the 15 models that participated in the WCRP workshop of 1995 (Rasch et al., 2000).

A further assessment of UKCA's wet scavenging is carried out by comparing modelled annual mean surface ${ }^{210} \mathrm{~Pb}$ deposition fluxes with the global gridded data set of Preiss and Genthon (1997). This data set is based on the previously compiled database by Preiss et al. (1996), except that it has been reduced and aggregated onto a $5^{\circ} \times 5^{\circ}$ grid, thus making it more convenient for model-observation comparisons. Figure 7 shows a scatter plot of HadGEM2-UKCA modelled multi-annual mean surface ${ }^{210} \mathrm{~Pb}$ deposition fluxes and observed fluxes. The correlation coefficient between modelled and measured deposition fluxes is fairly high at 0.69 . However, there is considerable scatter about the $1: 1$ line, indicating some large regional discrepancies (e.g. Northern Hemisphere tropics and mid-latitudes). Some of these could be related to precipitation biases in the climate model (Martin et al., 2006, 2010). However, they could also be related to regional variability in ${ }^{222} \mathrm{Rn}$ emissions not represented in the model simulation. Figure 8 shows the global distribution of the modelled global annual mean deposition terms from each of the deposition processes in addition to the total deposition obtained from the 5-yr simulation. The contributions from the various processes were found to be as follows: dry deposition $9.5 \%$, dynamic scavenging $17.8 \%$, and convective scavenging $72.7 \%$, and are reasonably consistent with other estimates (e.g. 14, 12, and $74 \%$ for dry deposition, dynamic scavenging, and convective scavenging, respectively, from Balkanski et al., 1993).

Guelle et al. (1998a) concluded that it is necessary to compare with vertical profiles of ${ }^{210} \mathrm{~Pb}$ to discriminate between different wet deposition schemes. As a result, we make use of ${ }^{210} \mathrm{~Pb}$ measurements taken onboard 16 flights of the NASA DC-8 aircraft during the Global Tropospheric Experiment/Pacific Exploratory Mission-West B (GTE/PEMWest B) campaign which took place during February and March 1994 (Dibb et al., 1997). Following Dibb et al. (1996), the measurements from PEM-West B were separated into two geographic regions: "remote Pacific" and "near Asia". For the purposes of comparing with the observations, multiannual February and March monthly mean concentrations from HadGEM2-UKCA were averaged over the regions: $10-30^{\circ} \mathrm{N}, 140-150^{\circ} \mathrm{E}$ and $20-45^{\circ} \mathrm{N}, 120-145^{\circ} \mathrm{E}$. Figure 9 

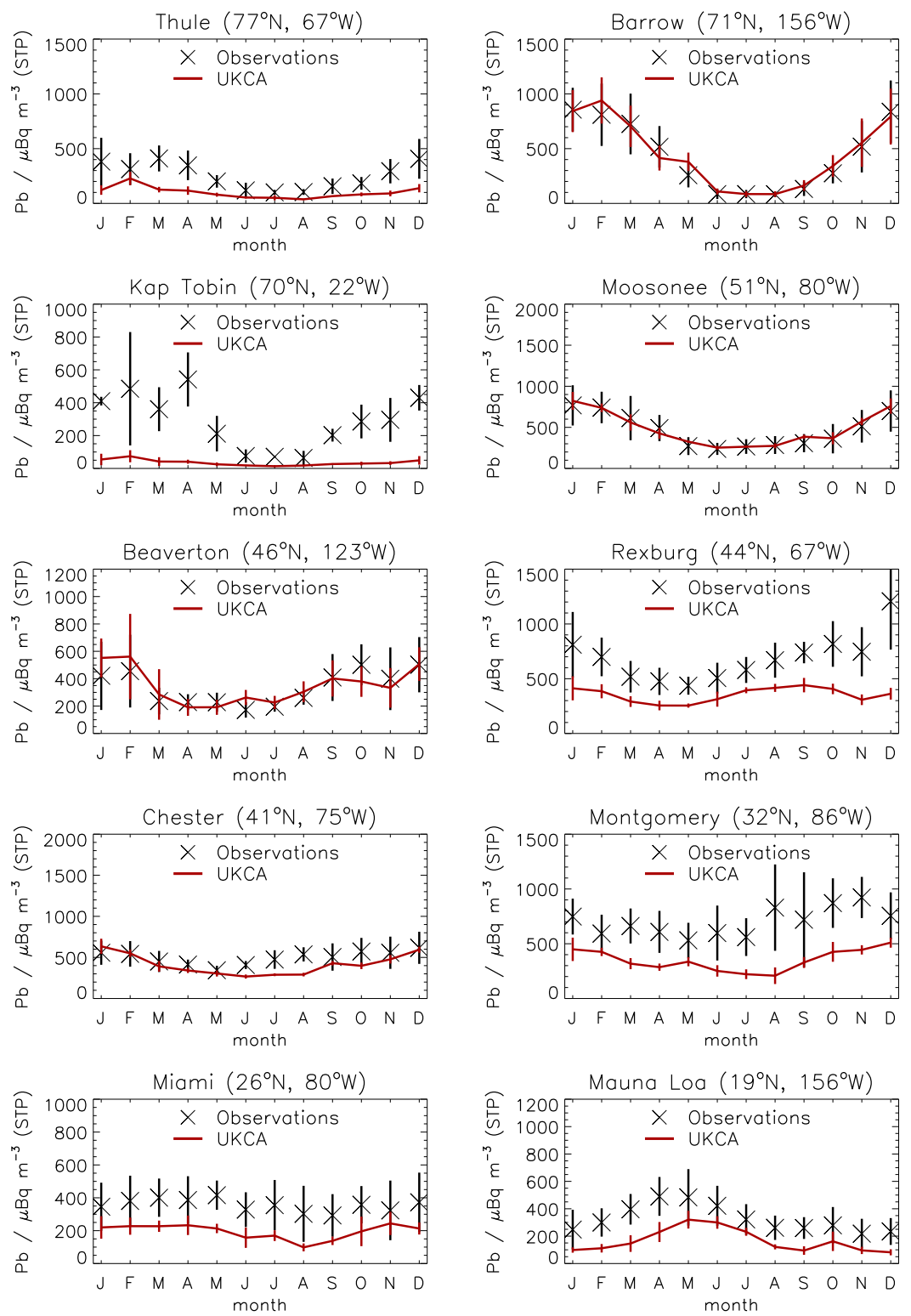

Fig. 5. Comparison of modelled surface lead concentrations in units of $\mu \mathrm{Bq} \mathrm{m}^{-3}$ (STP) with surface observations for a number of sites in the Northern Hemisphere. Observations are shown as black crosses and the UKCA model is shown in red. Measurements are taken from the Surface Air Sampling Program database of the Environmental Measurements Laboratory (EML) (http://esd.lbl.gov/research/facilities/eml/).

shows the comparison of HadGEM2-UKCA with the observed profiles over these regions. Given that the model is not running with the specific meteorology associated with PEM West-B, the comparison is only intended to be a general comparison of the calculated profiles. However, it is expected that the measured profiles lie within the variability (spatial and temporal) of the modelled profiles. For the "remote Pacific", HadGEM2-UKCA matches the absolute mean ${ }^{210} \mathrm{~Pb}$ concentrations and the shape of the observed profile particularly well. In particular, scavenging during PEMWest B was dominated by dynamic scavenging (Guelle et al., 1998a) and the results suggest that the climate model's dynamic precipitation and UKCA's wet scavenging scheme are performing well. For the "near Asia" comparison, the modelled concentrations and the shape of the modelled profile from HadGEM2-UKCA again more closely match those of the observations. In HadGEM2, three-dimensional precipitation diagnostics were specifically added to the climate model for the purpose of wet scavenging. These comparisons, albeit limited, suggest that the wet scavenging in HadGEM2UKCA by dynamic and convective precipitation is reasonable. Guelle et al. (1998a) concluded that the scheme used by 

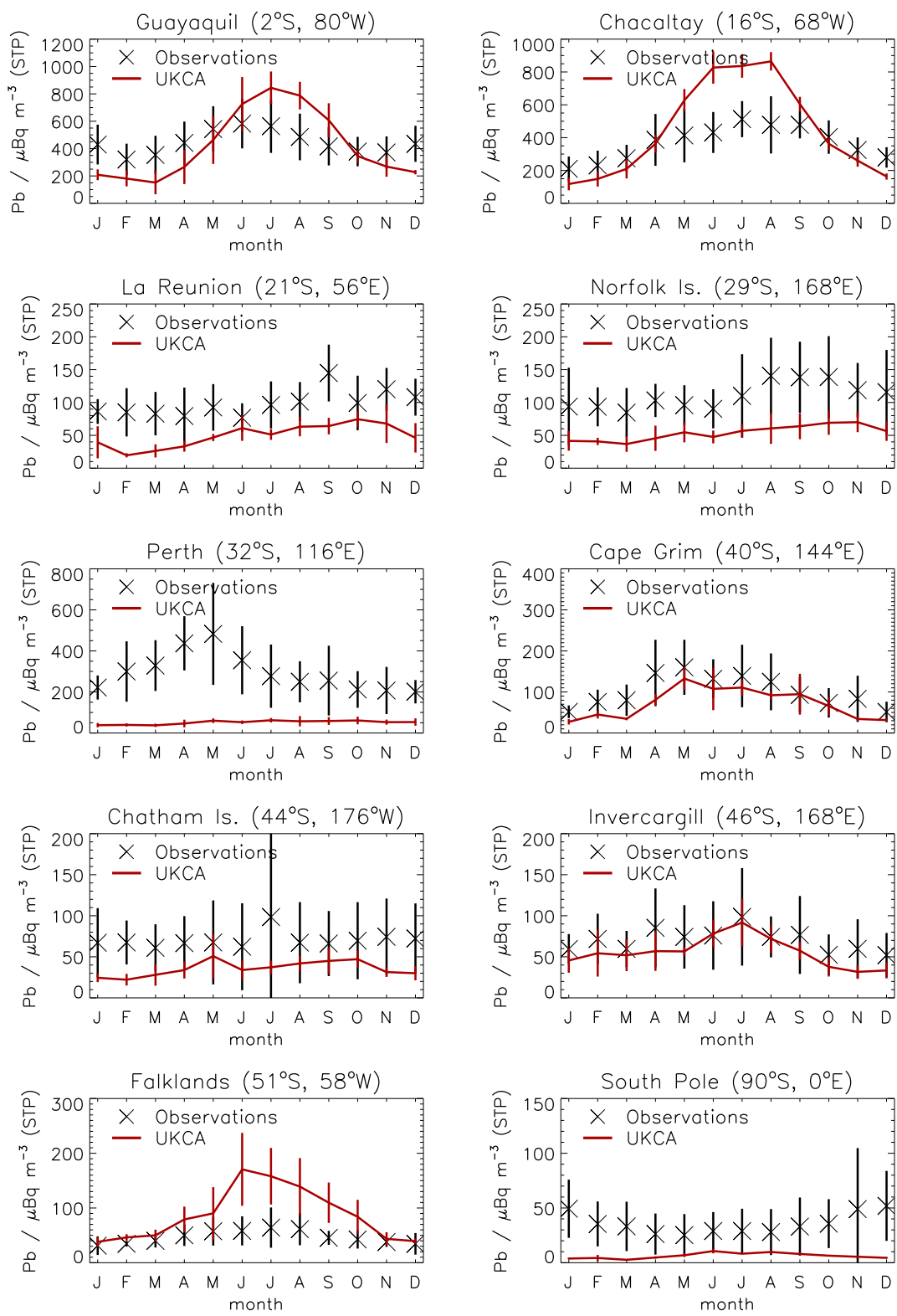

Fig. 6. Comparison of modelled surface lead concentrations in units of $\mu \mathrm{Bq} \mathrm{m}^{-3}$ (STP) with surface observations for a number of sites in the Southern Hemisphere. Observations are shown as black crosses and the UKCA model is shown in red. Measurements are taken from the Surface Air Sampling Program database of the Environmental Measurements Laboratory (EML) (http://esd.lbl.gov/research/facilities/eml/).

Balkanski et al. (1993) and extended by Guelle et al. (1998b) to take account of size-distributed aerosols was better than that of Walton et al. (1988). Although we have not tested multiple scavenging schemes within UKCA, these comparisons suggest that the performance of the Walton et al. (1988) scheme in HadGEM2-UKCA is satisfactory and comparable to that from other models (e.g. Guelle et al., 1998a; Hauglustaine et al., 2004).

\subsection{Global mass conservation}

By examining the time series of the accumulated burden of the second (non-deposited) ${ }^{210} \mathrm{~Pb}$ tracer and comparing it with the accumulated ${ }^{222} \mathrm{Rn}$ emissions, the global mass conservation of a tracer can be assessed. It was found that the rate of increase of this inert tracer was less than $0.008 \%$ per year compared to the accumulated emissions, indicating that the model drift due to global non-conservation of tracers is quite small. The degree of conservation was also assessed by comparing the emissions of ${ }^{222} \mathrm{Rn}$ with the deposition of the 


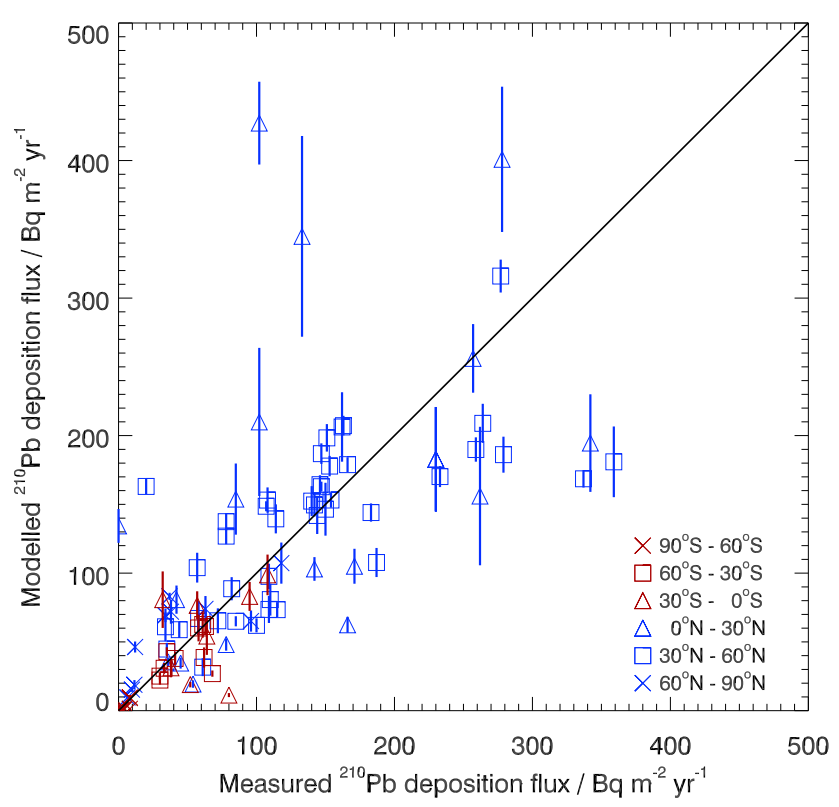

Fig. 7. Scatter plot of the modelled and measured ${ }^{210} \mathrm{~Pb}$ annual deposition fluxes in units of $\mathrm{Bqm}^{-2} \mathrm{yr}^{-1}$, where $1.0 \mathrm{Bqm}^{-2} \mathrm{yr}^{-1}$ is equivalent to $8.87 \times 10^{22} \mathrm{~kg} \mathrm{~m}^{-2} \mathrm{yr}^{-1}$. Northern Hemisphere points are shown in blue and Southern Hemisphere points are shown in red. The vertical whiskers represent \pm 1 standard deviation of the modelled annual mean deposition fluxes at each location. Measurements are taken from Preiss and Genthon (1997).

deposited ${ }^{210} \mathrm{~Pb}$ tracer. In this case, the difference is of the order of $5 \%$, comparable to what is found for aerosol schemes in the same climate model (e.g. Bellouin et al., 2011).

\subsection{Inter-hemispheric transport}

With the majority of anthropogenic emissions originating in the Northern Hemisphere, inter-hemispheric transport plays an important role in redistributing pollutants from the Northern to the Southern Hemisphere. A model's ability to simulate inter-hemispheric transport accurately and with the correct timescale has implications for modelling meridional gradients of long-lived tracers and the accuracy of source estimates by inverse modelling (e.g. Houweling et al., 1999; Chen and Prinn, 2006). Meridional surface observations of long-lived tracers of Northern Hemisphere origin have been used to estimate the timescale for inter-hemispheric exchange (e.g. Weiss et al., 1983). However, estimates from three-dimensional fields tend to be more accurate (Jacob et al., 1987; Levin and Hesshaimer, 1996; Denning et al., 1999). The timescale for inter-hemispheric transport from a number of model studies has been estimated to be of the order of one year (Prather et al., 1987; Jacob et al., 1987; Rind and Lerner, 1996; Levin and Hesshaimer, 1996) but intercomparisons indicate that models reproduce this timescale with varying degrees of success (e.g. $0.55-1.26 \mathrm{yr}$ from three-dimensional fields in Denning et al., 1999).

A number of different long-lived tracers, such as CFCs, sulphur hexafluoride $\left(\mathrm{SF}_{6}\right)$, and Krypton- $85\left({ }^{85} \mathrm{Kr}\right)$, have been used to assess inter-hemispheric transport. Here, we use the classical ${ }^{85} \mathrm{Kr}$ experiment, as discussed by Jacob et al. (1987), to estimate the timescale for interhemispheric exchange in the HadGEM2-UKCA coupled model. ${ }^{85} \mathrm{Kr}$, produced during reprocessing of nuclear fuel, was emitted from seven known point sources and one estimated point source in the Northern Hemisphere during the period 1978-1983. The integration itself was started on 1 September 1978 with initial mass mixing ratios taken from Rind and Lerner (1996) and scaled to give a global burden in $\mathrm{kg}$ of $M=166.2+5.10 \times(T-1980.0)$, where $T$ is the initialisation time in yr. In this way, the initial global burden agreed with that estimated by Jacob et al. (1987). Sea surface temperatures, sea ice distributions, and emissions were prescribed during the integration according to the date but the model dynamics were free-running. The only sink of ${ }^{85} \mathrm{Kr}$ was radioactive decay to Rubidium- $85\left({ }^{85} \mathrm{Rb}\right)$ with an e-folding lifetime of $15.52 \mathrm{yr}$.

Before inter-hemispheric transport can be assessed, the model simulation itself was verified against that of Jacob et al. (1987). For example, it was found that the rate of change of the global burden of ${ }^{85} \mathrm{Kr}$ during the $1978-$ 1983 period was comparable to that of Jacob et al. (1987). HadGEM2-UKCA model results show that in the Northern Hemisphere winter, there are extensive areas north of $45^{\circ} \mathrm{N}$ with surface concentrations in excess of $23 \mathrm{pCim}^{-3}$ at STP. By contrast, in the Northern Hemisphere summer, modelled concentrations greater than $23 \mathrm{pCim}^{-3}$ at STP were only found over a limited region within Europe. These wintertime maxima can largely be attributed to the lack of convective activity in the Northern Hemisphere winter, resulting in high ${ }^{85} \mathrm{Kr}$ plumes being vertically confined to the lower troposphere. This winter-summer contrast was also evident in the ${ }^{85} \mathrm{Kr}$ model results of Jacob et al. (1987) and Hauglustaine et al. (2004), and in other modelling studies (e.g. Prather et al., 1987; Wang and Shallcross, 2000).

The HadGEM2-UKCA coupled model results can be further verified by comparison with observations. Nine meridional profiles of ${ }^{85} \mathrm{Kr}$ were measured during ship cruises in the Atlantic Ocean between 1980 and 1987 by W. Weiss and reported by Jacob et al. (1987). Model results are shown for two longitude bands $\left(7.5\right.$ and $\left.30^{\circ} \mathrm{W}\right)$ which bracket the measurements. The comparison in March (Fig. 10a) shows that HadGEM2-UKCA captures the homogeneous meridional profile in the Southern Hemisphere and the more spatially variable profile in the Northern Hemisphere indicated by the observations. The model's meridional gradient seems quite robust in all model years and occurs at approximately $0^{\circ} \mathrm{N}$. This matches quite well with the observed tropical gradient. The comparison for October (Fig. 10b) shows that there is a good correspondence between modelled and measured 

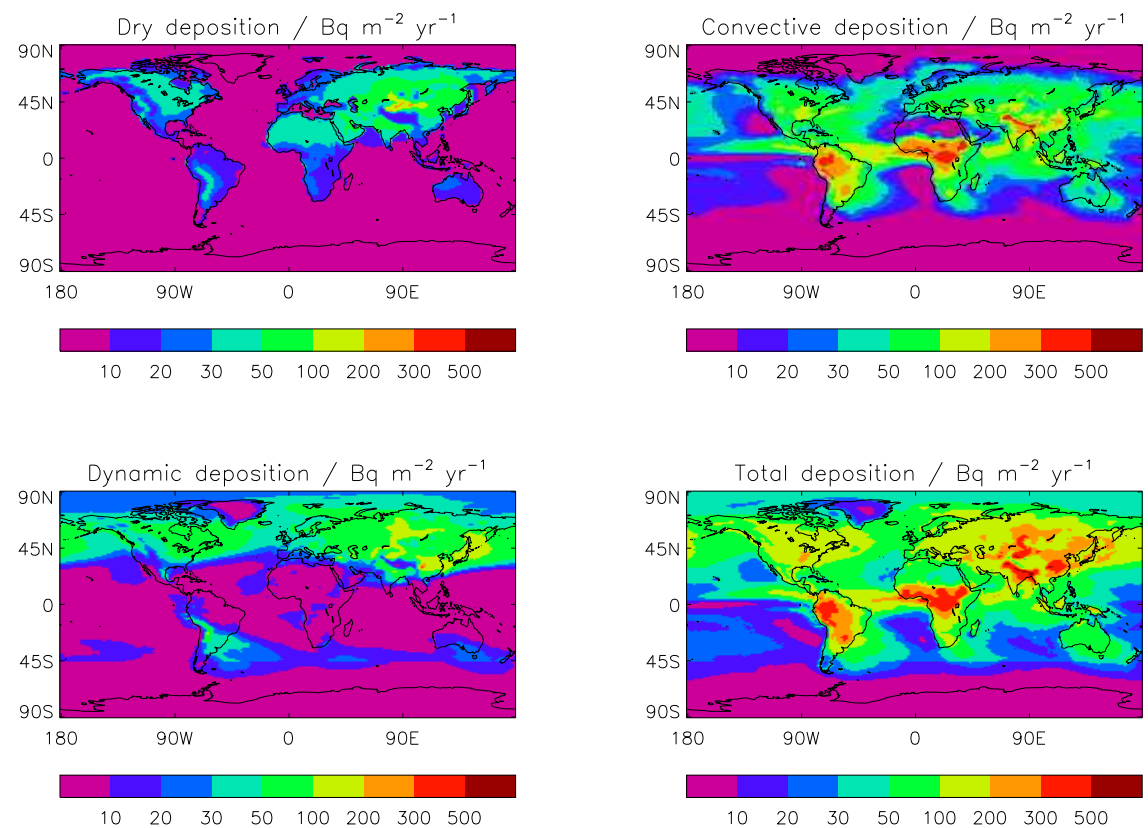

Fig. 8. Global multi-annual mean distribution of ${ }^{210} \mathrm{~Pb}$ deposition fluxes from UKCA: Dry deposition, convective scavenging, dynamic scavenging, and total deposition flux. Units are in $\mathrm{Bqm}^{-2} \mathrm{yr}^{-1}$.
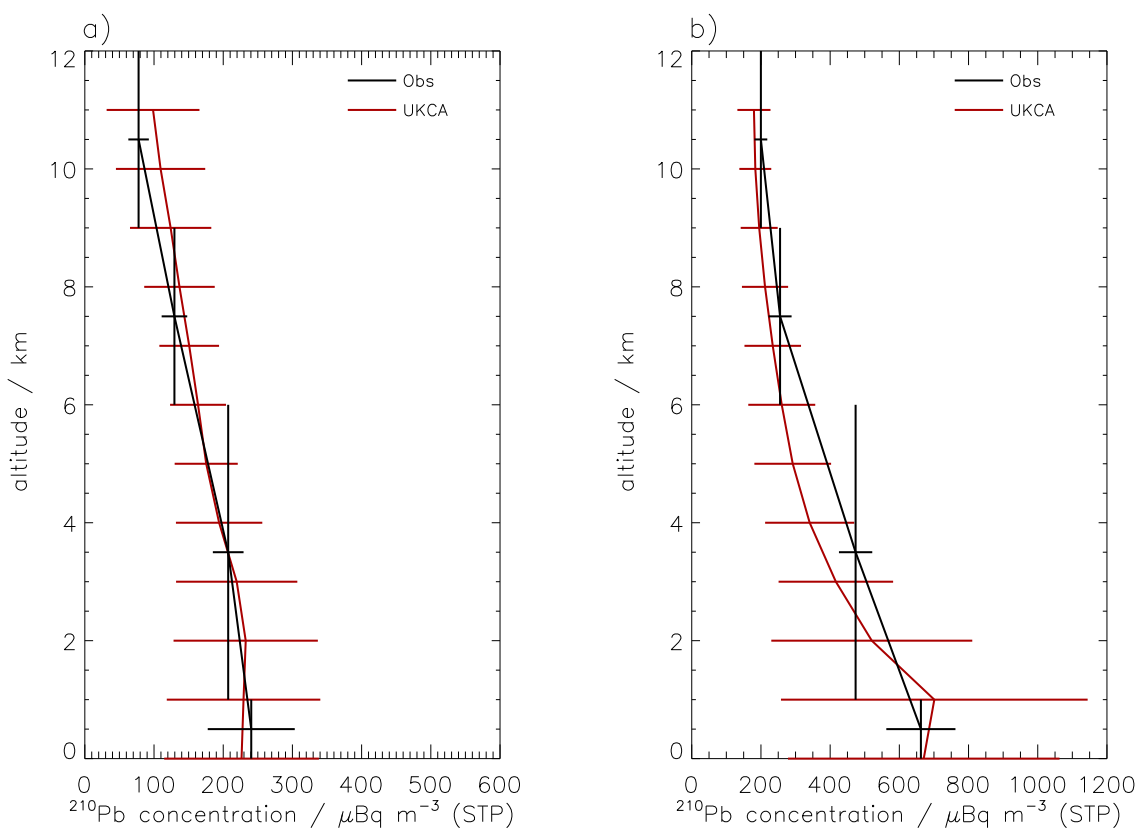

Fig. 9. Comparison between observed vertical profiles of ${ }^{210} \mathrm{~Pb}$ concentrations with modelled concentrations from UKCA (red) over (a) the "remote Pacific" and (b) over "near Asia". Observations are taken from Dibb et al. (1997).

concentrations for October 1980, suggesting that the initial mass mixing ratio field used was reasonable. However, the tropical gradient in the model is shifted equatorward by about $10^{\circ}$ in comparison with the observations. This was also the case in the evaluation of the LMDz-INCA model by Hauglustaine et al. (2004), and was attributed to a reduced seasonal cycle in the Intertropical Convergence Zone (ITCZ) location. For October 1983, modelled concentrations in the Southern Hemisphere appear too high, the tropical gradient is again shifted equatorward, and the low observed 

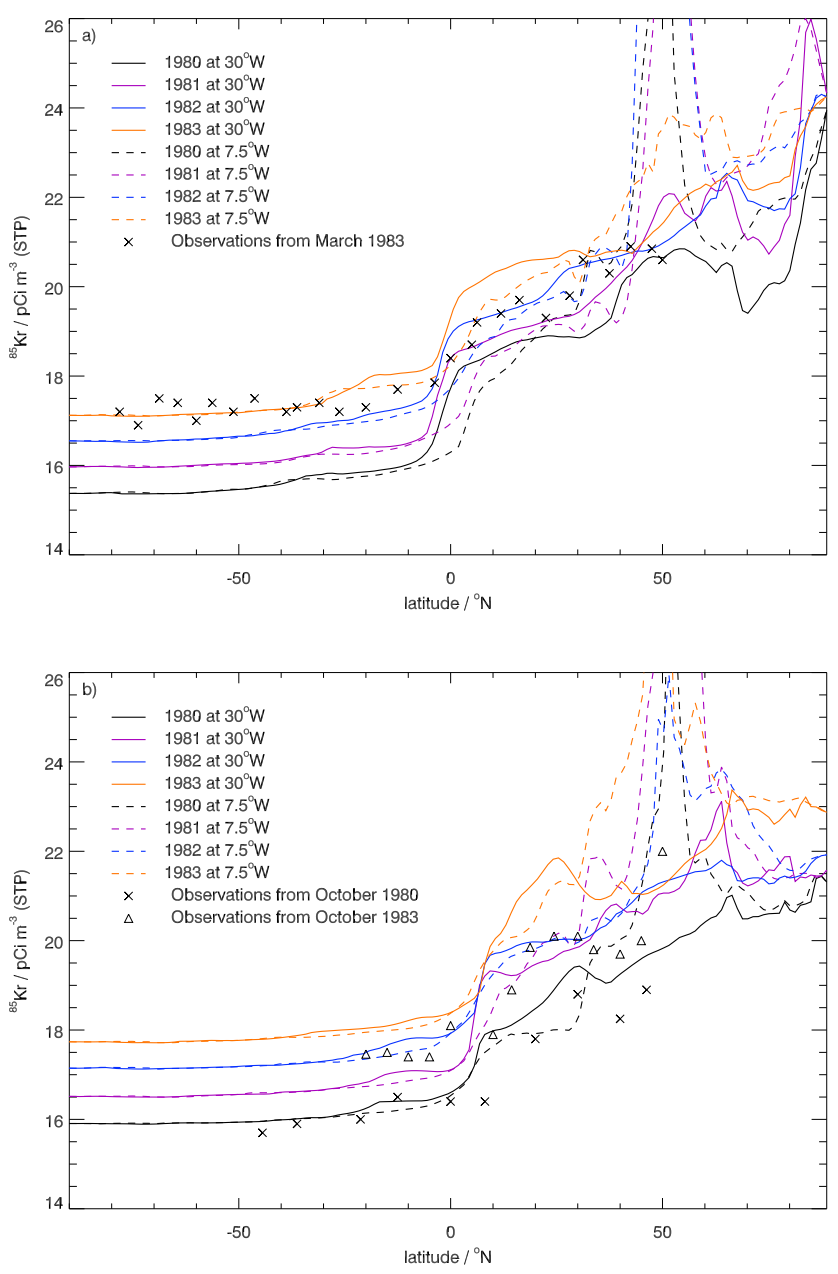

Fig. 10. Comparison between observed meridional profiles of ${ }^{85} \mathrm{Kr}$ concentrations with modelled concentrations from UKCA over the Atlantic ocean (a) in March and (b) in October. Observations are taken from Weiss et al. (1983) and UKCA was sampled at two longitudes $\left(7.5\right.$ and $\left.30^{\circ} \mathrm{W}\right)$ to straddle the area sampled by the observations.

concentrations at $40^{\circ} \mathrm{N}$ are not well simulated. Nevertheless, the modelled inter-hemispheric difference in the surface concentrations matches the observed inter-hemispheric difference, and as a result, the modelled three-dimensional concentrations can be used to evaluate a timescale for interhemispheric exchange.

In an idealised two-box model, the inter-hemispheric difference in mean concentration, $\mathrm{C}_{\mathrm{ns}}$, evolves with time according to Jacob et al. (1987):

$\frac{\mathrm{d} C_{\mathrm{ns}}}{\mathrm{d} t}=\frac{E}{1.96}-C_{\mathrm{ns}} \times\left(2 t_{\mathrm{e}}^{-1}+t_{\mathrm{d}}^{-1}\right)$,

where $C_{\mathrm{ns}}=C_{\mathrm{n}}-C_{\mathrm{s}}$ is the difference between the mean northern and southern hemispheric concentrations (in $\mathrm{pCim}^{-3}$ at STP), $E$ is the emission rate (in $\mathrm{MCiyr}^{-1}$ ), $t_{\mathrm{e}}$ is the inter-hemispheric transport timescale (in $\mathrm{yr}$ ), $t_{\mathrm{d}}$ is the e-folding lifetime (in yr) and the 1.96 is a conversion factor to relate a mean hemispheric concentration in $\mathrm{pCi} \mathrm{m}^{-3}$ (STP) to a hemispheric inventory in $\mathrm{MCi}$. If $C_{\mathrm{ns}}$ reaches a steady state, then the inter-hemispheric transport timescale can be computed from the steady state value of $C_{\mathrm{ns}}$ as follows:

$t_{e}=\left(\frac{E}{3.92 \times C_{\mathrm{ns}}}-\frac{1}{2 t_{\mathrm{d}}}\right)^{-1}$.

In this experiment, $C_{\mathrm{ns}}$ was reasonably stable throughout the integration, i.e. the slope of a least squares linear fit to the time series of $C_{\mathrm{ns}}$ was $-0.02 \mathrm{pCim}^{-3}$ (STP) $\mathrm{yr}^{-1}$ with a mean value for $C_{\mathrm{ns}}$ of $2.01 \mathrm{pCim}^{-3}$ (STP) during the years 1979-1983. Taking a mean emission rate of $6.33 \mathrm{MCi} \mathrm{yr}^{-1}$ for the years 1978-1983, the inter-hemispheric transport timescale was estimated to be $1.3 \mathrm{yr}$. This model-derived timescale is larger than the value of $1.1 \mathrm{yr}$ estimated by Jacob et al. (1987) and at the upper end of the range of estimates from Denning et al. (1999) but close to that estimated from $\mathrm{CFC}$ and ${ }^{85} \mathrm{Kr}$ experiments in the Goddard Institute for Space Studies (GISS) model by Rind and Lerner (1996).

Prather et al. (1987) found that cross-equatorial transport occurs primarily in the tropical upper troposphere and is associated with deep convection. The mechanistic analysis by Hartley and Black (1995) also found that convective outflow rather than Rossby waves was the dominant mechanism for cross-equatorial transport and is supported by the more recent study of Lintner et al. (2004). However, the model intercomparison of $\mathrm{SF}_{6}$ simulations by Denning et al. (1999) found that models which compare favourably with observations in the remote marine boundary layer tended to overestimate observed concentrations at Northern Hemisphere continental sites. They deduced that boundary layer mixing rather than meridional transport may be the dominant control on simulating the meridional gradient of long-lived tracers of Northern Hemisphere origin. Given this sensitivity to boundary layer mixing, Law and Corbin (2009), using an atmosphere-only version of HadGEM2 at N48L38, tested the sensitivity of carbon dioxide $\left(\mathrm{CO}_{2}\right)$ and $\mathrm{SF}_{6}$ transport to the stability dependence of the vertical transport in the stable boundary layer. Irrespective of their choice of function for parameterizing mixing within a stable boundary layer, the inter-hemispheric difference in $\mathrm{SF}_{6}$ was still too large and they inferred that inter-hemispheric transport in the N48 version of HadGEM2 was too slow. A simulation of ${ }^{85} \mathrm{Kr}$ with UKCA at a horizontal resolution of N48 gave identical values to those computed for N96 above and confirms their conclusions. Moreover, Martin et al. (2006) found that in HadGAM1, meridional winds, particularly at upper levels, were worse and there was less boundary layer mixing at a given stability than in its predecessor, HadCM3 (Gordon et al., 2000). Other contributing factors to the slow interhemispheric transport in HadGEM2-UKCA may be insufficient mixing within unstable boundary layers and/or deficiencies in tropical deep convection. 


\section{Tropospheric chemistry description}

\subsection{Chemistry schemes}

Included in this description of the tropospheric configuration of the UKCA model are two chemistry schemes. The first chemistry scheme, called StdTrop, includes a relatively thorough description of inorganic odd oxygen $\left(\mathrm{O}_{\mathrm{x}}\right)$, nitrogen $\left(\mathrm{NO}_{\mathrm{y}}\right)$, hydrogen $\left(\mathrm{HO}_{\mathrm{x}},=\mathrm{OH}+\mathrm{HO}_{2}\right)$, and carbon monoxide $(\mathrm{CO})$ chemistry with near-explicit treatment of methane $\left(\mathrm{CH}_{4}\right)$, ethane $\left(\mathrm{C}_{2} \mathrm{H}_{6}\right)$, propane $\left(\mathrm{C}_{3} \mathrm{H}_{8}\right)$, and acetone $\left(\mathrm{CH}_{3} \mathrm{COCH}_{3}\right)$ degradation (including formaldehyde ( $\mathrm{HCHO})$, acetaldehyde $\left(\mathrm{CH}_{3} \mathrm{CHO}\right)$, peroxy acetyl nitrate (PAN), and peroxy propionyl nitrate (PPAN)). It makes use of 25 tracers and represents 41 species, as indicated in Table 3. The atmospheric constituents hydrogen $\left(\mathrm{H}_{2}\right), \mathrm{CO}_{2}$, oxygen $\left(\mathrm{O}_{2}\right)$, and nitrogen $\left(\mathrm{N}_{2}\right)$ have fixed global concentrations. We ignore chemical production and loss of water vapour; instead, it is taken from the climate model on a timestep-by-timestep basis. All remaining non-advected species are treated in steady state and their concentrations are retained from one timestep to the next.

This chemistry scheme accounts for 25 photolytic reactions (Table 4), 83 bimolecular reactions (Table 5), and 13 uni- and termolecular reactions (Table 6). Reaction rate coefficients are taken from the 2000-2005 recommendations provided by the International Union of Pure and Applied Chemistry (IUPAC) Subcommittee for Gas Kinetic Data Evaluation (http://www.iupac-kinetic.ch.cam.ac.uk/), versions 2 and 3.1 of the Master Chemical Mechanism (MCM) (Jenkin et al., 1997, 2003; Saunders et al., 1997, 2003; Bloss et al., 2005; Tyndall et al., 2001; Ravishankara et al., 2002). This scheme has previously been used in both the TOMCAT CTM (e.g. Law et al., 1998; Savage et al., 2004) and chemistry-climate integrations (e.g. Zeng and Pyle, 2003, 2005). The non-methane VOCs (NMVOCs) included in StdTrop were found by Saunders et al. (2003) to have low photochemical ozone creation potential (POCP) values (Derwent and Jenkin, 1991) (i.e. in the range of 7.5-55.0) and, as a result, the StdTrop scheme even in background conditions will be deficient in its representation of tropospheric chemistry (e.g. Mickley et al., 2001).

Alkenes (including dienes) generally have the highest POCP values. Isoprene $\left(\mathrm{C}_{5} \mathrm{H}_{8}\right)$, for instance, has a POCP value of 101.6, compared to 8.8 for $\mathrm{C}_{2} \mathrm{H}_{6}$ (Saunders et al., 2003), although its impact on tropospheric $\mathrm{O}_{3}$ depends on the environment (e.g. Young et al., 2009). Wang et al. (1998) has also shown that $\mathrm{C}_{5} \mathrm{H}_{8}$ alone accounts for half of PAN formation and half of the effects of all NMVOCs on $\mathrm{NO}_{\mathrm{x}}, \mathrm{OH}$, and $\mathrm{O}_{3}$. Emissions of $\mathrm{C}_{5} \mathrm{H}_{8}$ are comparable in magnitude to $\mathrm{CH}_{4}$ emissions (Lamb et al., 1993). As a result, the second tropospheric chemistry scheme implemented in the tropospheric configuration of UKCA, called TropIsop, is an extension of StdTrop to include the extra species and reactions of the lumped $\mathrm{C}_{5} \mathrm{H}_{8}$ oxidation scheme
Table 3. Tracers/species in the UKCA standard tropospheric (StdTrop) scheme, where $\mathrm{Me}=\mathrm{CH}_{3}, \mathrm{Et}=\mathrm{C}_{2} \mathrm{H}_{5}, \mathrm{Pr}=\mathrm{C}_{3} \mathrm{H}_{7}$, $\mathrm{PAN}=$ peroxy acetyl nitrate, and PPAN $=$ peroxy propionyl nitrate.

\begin{tabular}{|c|c|}
\hline Species Name & Treatment \\
\hline $\mathrm{H}_{2} \mathrm{O}$ & Constant Field \\
\hline $\mathrm{CO}_{2}$ & Constant Tracer \\
\hline $\mathrm{H}_{2}$ & Constant Tracer \\
\hline $\mathrm{N}_{2}$ & Constant Tracer \\
\hline $\mathrm{O}_{2}$ & Constant Tracer \\
\hline $\mathrm{O}(3 \mathrm{P})$ & Steady State \\
\hline O(1D) & Steady State \\
\hline $\mathrm{OH}$ & Steady State \\
\hline $\mathrm{HO}_{2}$ & Steady State \\
\hline $\mathrm{MeOO}$ & Steady State \\
\hline EtOO & Steady State \\
\hline $\mathrm{MeCO}_{3}$ & Steady State \\
\hline$n$-PrOO & Steady State \\
\hline$i$-PrOO & Steady State \\
\hline $\mathrm{EtCO} 3$ & Steady State \\
\hline $\mathrm{MeCOCH}_{2} \mathrm{OO}$ & Steady State \\
\hline $\mathrm{O}_{3}$ & Transported Tracer \\
\hline NO & Transported Tracer \\
\hline $\mathrm{NO}_{3}$ & Transported Tracer \\
\hline $\mathrm{NO}_{2}$ & Transported Tracer \\
\hline $\mathrm{N}_{2} \mathrm{O}_{5}$ & Transported Tracer \\
\hline $\mathrm{HO}_{2} \mathrm{NO}_{2}$ & Transported Tracer \\
\hline $\mathrm{HONO}_{2}$ & Transported Tracer \\
\hline $\mathrm{H}_{2} \mathrm{O}_{2}$ & Transported Tracer \\
\hline $\mathrm{CH}_{4}$ & Transported Tracer \\
\hline $\mathrm{CO}$ & Transported Tracer \\
\hline $\mathrm{HCHO}$ & Transported Tracer \\
\hline $\mathrm{MeOOH}$ & Transported Tracer \\
\hline HONO & Transported Tracer \\
\hline $\mathrm{C}_{2} \mathrm{H}_{6}$ & Transported Tracer \\
\hline EtOOH & Transported Tracer \\
\hline $\mathrm{MeCHO}$ & Transported Tracer \\
\hline PAN & Transported Tracer \\
\hline $\mathrm{C}_{3} \mathrm{H}_{8}$ & Transported Tracer \\
\hline$n$-PrOOH & Transported Tracer \\
\hline$i-\mathrm{PrOOH}$ & Transported Tracer \\
\hline EtCHO & Transported Tracer \\
\hline $\mathrm{Me}_{2} \mathrm{CO}$ & Transported Tracer \\
\hline $\mathrm{MeCOCH}_{2} \mathrm{OOH}$ & Transported Tracer \\
\hline PPAN & Transported Tracer \\
\hline $\mathrm{MeONO}_{2}$ & Transported Tracer \\
\hline
\end{tabular}

from the Mainz Isoprene Mechanism (MIM) described by Pöschl et al. (2000). It includes an additional 14 organic species (Table 7), 12 of which are treated as tracers with the remainder treated in steady state. The additional 10 photolytic, 31 bimolecular, and 2 uni- and termolecular reactions can be found in Tables 8-10. Reaction rate coefficients are taken from the 2005 recommendations provided by the International Union of Pure and Applied Chemistry (IUPAC) Subcommittee for Gas Kinetic Data Evaluation 
Table 4. Photolytic reactions in the UKCA standard tropospheric (StdTrop) scheme, where $\mathrm{Me}=\mathrm{CH}_{3}, \mathrm{Et}=\mathrm{C}_{2} \mathrm{H}_{5}, \mathrm{Pr}=\mathrm{C}_{3} \mathrm{H}_{7}$, $\mathrm{PAN}=$ peroxy acetyl nitrate, and $\mathrm{PPAN}=$ peroxy propionyl nitrate.

\begin{tabular}{|c|c|}
\hline Label & Reaction \\
\hline 1 & $\mathrm{EtOOH}+h v \rightarrow \mathrm{MeCHO}+\mathrm{HO}_{2}+\mathrm{OH}$ \\
\hline 2 & $\mathrm{H}_{2} \mathrm{O}_{2}+h v \rightarrow \mathrm{OH}+\mathrm{OH}$ \\
\hline 3 & $\mathrm{HCHO}+h v \rightarrow \mathrm{HO}_{2}+\mathrm{HO}_{2}+\mathrm{CO}$ \\
\hline 4 & $\mathrm{HCHO}+h v \rightarrow \mathrm{H}_{2}+\mathrm{CO}$ \\
\hline 5 & $\mathrm{HO}_{2} \mathrm{NO}_{2}+h v \rightarrow \mathrm{HO}_{2}+\mathrm{NO}_{2}$ \\
\hline 6 & $\mathrm{HONO}_{2}+h v \rightarrow \mathrm{OH}+\mathrm{NO}_{2}$ \\
\hline 7 & $\mathrm{MeCHO}+h v \rightarrow \mathrm{MeOO}+\mathrm{HO}_{2}+\mathrm{CO}$ \\
\hline 8 & $\mathrm{MeCHO}+h v \rightarrow \mathrm{CH}_{4}+\mathrm{CO}$ \\
\hline 9 & $\mathrm{MeOOH}+h v \rightarrow \mathrm{HO}_{2}+\mathrm{HCHO}+\mathrm{OH}$ \\
\hline 10 & $\mathrm{~N}_{2} \mathrm{O}_{5}+h v \rightarrow \mathrm{NO}_{3}+\mathrm{NO}_{2}$ \\
\hline 11 & $\mathrm{NO}_{2}+h v \rightarrow \mathrm{NO}+\mathrm{O}(3 \mathrm{P})$ \\
\hline 12 & $\mathrm{NO}_{3}+h v \rightarrow \mathrm{NO}+\mathrm{O}_{2}$ \\
\hline 13 & $\mathrm{NO}_{3}+h v \rightarrow \mathrm{NO}_{2}+\mathrm{O}(3 \mathrm{P})$ \\
\hline 14 & $\mathrm{O}_{2}+h v \rightarrow \mathrm{O}(3 \mathrm{P})+\mathrm{O}(3 \mathrm{P})$ \\
\hline 15 & $\mathrm{O}_{3}+h v \rightarrow \mathrm{O}_{2}+\mathrm{O}(1 \mathrm{D})$ \\
\hline 16 & $\mathrm{O}_{3}+h v \rightarrow \mathrm{O}_{2}+\mathrm{O}(3 \mathrm{P})$ \\
\hline 17 & $\mathrm{PAN}+h v \rightarrow \mathrm{MeCO}_{3}+\mathrm{NO}_{2}$ \\
\hline 18 & $\mathrm{HONO}+h v \rightarrow \mathrm{OH}+\mathrm{NO}$ \\
\hline 19 & $\mathrm{EtCHO}+h v \rightarrow \mathrm{EtOO}+\mathrm{HO}_{2}+\mathrm{CO}$ \\
\hline 20 & $\mathrm{Me}_{2} \mathrm{CO}+h v \rightarrow \mathrm{MeCO}_{3}+\mathrm{MeOO}$ \\
\hline 21 & $n-\mathrm{PrOOH}+h v \rightarrow \mathrm{EtCHO}+\mathrm{HO}_{2}+\mathrm{OH}$ \\
\hline 22 & $i-\mathrm{PrOOH}+h v \rightarrow \mathrm{Me}_{2} \mathrm{CO}+\mathrm{HO}_{2}+\mathrm{OH}$ \\
\hline 23 & $\mathrm{MeCOCH}_{2} \mathrm{OOH}+h v \rightarrow \mathrm{MeCO}_{3}+\mathrm{HCHO}+\mathrm{OH}$ \\
\hline 24 & $\mathrm{PPAN}+h v \rightarrow \mathrm{EtCO}_{3}+\mathrm{NO}_{2}$ \\
\hline 25 & $\mathrm{MeONO}_{2}+h v \rightarrow \mathrm{HO}_{2}+\mathrm{HCHO}+\mathrm{NO}_{2}$ \\
\hline
\end{tabular}

(http://www.iupac-kinetic.ch.cam.ac.uk/), version 3 of the MCM (Saunders et al., 2003; Jenkin et al., 2003), and Pöschl et al. (2000) and references therein. Most of the reactions from the MIM are combinations of the major MCM reaction pathways and the additional species represent different classes of compounds as described in Table 7. This scheme has previously been used in both the p-TOMCAT CTM (Voulgarakis et al., 2009, 2010) and in chemistry-climate integrations (e.g. Zeng et al., 2008; Young et al., 2009).

In the Pöschl et al. (2000) study, the MIM was found to perform well in comparison with MCMv2 (Jenkin et al., 1997; Saunders et al., 1997); the two mechanisms agreed to within $10 \%$ for $\mathrm{O}_{3}$, hydroxyl $(\mathrm{OH}), \mathrm{CO}$, methyl hydroperoxide $(\mathrm{MeOOH})$, and PAN. The TropIsop chemistry scheme, as implemented in UKCA, was also included in a chemical mechanism intercomparison by Emmerson and Evans (2009); the scheme (referred to as TOMCAT in Emmerson and Evans, 2009) was found to show good agreement with MCMv3.1 in simulating both $\mathrm{O}_{3}$ and $\mathrm{OH}$ concentrations, even though all the carbon not represented explicitly in the scheme was lumped into $\mathrm{C}_{3} \mathrm{H}_{8}$. More recently, Archibald et al. (2010) carried out an intercomparison of $\mathrm{C}_{5} \mathrm{H}_{8}$ oxidation mechanisms, and found that all existing mechanisms, including MCMv3.1, cannot generate/recycle $\mathrm{HO}_{\mathrm{x}}$ at the rates needed to match reported radical observations from high $\mathrm{C}_{5} \mathrm{H}_{8} /$ low $\mathrm{NO}_{\mathrm{x}}$ environments (Lelieveld et al., 2008; Butler et al., 2008). In addition to insufficient $\mathrm{HO}_{\mathrm{x}}$ recycling, other uncertainties in the treatment of $\mathrm{C}_{5} \mathrm{H}_{8}$ in chemistry models include the strength of the global emission source (e.g. Arneth et al., 2008), chemical lumping (Archibald et al., 2010), treatment of isoprene nitrates (Pöschl et al., 2000; von Kuhlmann et al., 2004), and deposition of intermediate products (von Kuhlmann et al., 2004). Although not considered here, the TropIsop chemistry scheme has also been extended to include sulphur oxidation and a parameterization for monoterpene oxidation, thus enabling coupling to the aerosol component of the UKCA model, GLOMAP-mode. A description and evaluation of GLOMAP-mode, as implemented in a CTM, can be found in Mann et al. (2010). Part 3 of this series (Mann et al., 2014) will discuss the implementation and evaluation of GLOMAP-mode in a global climate model configuration.

The two schemes above, StdTrop and TropIsop, do not include higher NMVOCs than $\mathrm{C}_{3} \mathrm{H}_{8}$ and $\mathrm{C}_{5} \mathrm{H}_{8}$. However, details of an extension to TropIsop, called ExtTC, which considers the degradation pathways of $\mathrm{C} 2$ and $\mathrm{C} 3$ alkenes, $\mathrm{C} 4+$ alkanes, terpenes, and aromatics for use in chemistry-climate integrations can be found in Part 4 of the UKCA series by Folberth et al. (2014). Furthermore, a chemistry mechanism which considers some higher NMVOCs than $\mathrm{C}_{3} \mathrm{H}_{8}$ and $\mathrm{C}_{5} \mathrm{H}_{8}$ has been implemented in a regional configuration of UKCA for air quality purposes and is discussed in a separate publication (Savage et al., 2013).

Model studies suggest that the heterogeneous loss of the nitrate radical and dinitrogen pentoxide $\left(\mathrm{N}_{2} \mathrm{O}_{5}\right)$ can substantially reduce the tropospheric $\mathrm{NO}_{\mathrm{x}}$ burden (Dentener and Crutzen, 1993; Evans and Jacob, 2005), and that heterogeneous loss of the hydroperoxy radical $\left(\mathrm{HO}_{2}\right)$ can substantially impact tropospheric $\mathrm{HO}_{\mathrm{x}}$ (Mao et al., 2010). However, Shindell et al. (2009) argued that these heterogeneous loss processes only have a very small impact on the radiatively active species $\mathrm{O}_{3}$ and $\mathrm{CH}_{4}$. However, development work to introduce a parameterization for the uptake of both $\mathrm{N}_{2} \mathrm{O}_{5}$ and $\mathrm{HO}_{2}$ onto aerosols from the GLOMAP-mode scheme (Macintyre and Evans, 2010, 2011) has been completed and will be implemented in a future UKCA chemistry scheme.

The chemical equations in UKCA can be solved using one of two chemical solvers. The first one is an explicit iterative backward Euler approach (Hertel et al., 1993) with a chemical timestep of $5 \mathrm{~min}$ as is used in the STOCHEM model (e.g. Johnson et al., 1999; Collins et al., 2002). Alternatively, the chemistry can be treated in a more flexible way using the ASAD framework (Carver et al., 1997), built around a symbolic non-families sparse-matrix Newton-Raphson solver derived from Wild and Prather (2000); it is described in Morgenstern et al. (2009). In this paper, and in the sensitivity study of climate model biases by O'Connor et al. (2009), all model results were derived from the former approach. 
Table 5. Bimolecular reactions in the UKCA standard tropospheric (StdTrop) scheme, where $\mathrm{Me}_{2} \mathrm{CH}_{3}, \mathrm{Et}_{2}=\mathrm{C}_{2} \mathrm{H}_{5}, \mathrm{Pr}_{2}=\mathrm{C}_{3} \mathrm{H}_{7}$, PAN = peroxy acetyl nitrate, PPAN = peroxy propionyl nitrate, and MGLY = methylglyoxal. The rate coefficient of a bimolecular reaction is given by $k=A\left(\frac{T}{300}\right)^{\alpha} \exp \left(\frac{-E_{\mathrm{a}}}{R T}\right)$, where $A$ is the pre-exponential factor, $T$ is the temperature, $R$ is the gas constant, $\alpha$ is the exponent for the $\left(\frac{T}{300}\right)$ temperature dependence, and $E_{\mathrm{a}}$ is the Arrhenius activation energy. References: (1) Atkinson et al. (2000), (2) Atkinson et al. (2003), (3) Atkinson et al. (2004), (4) Atkinson et al. (2005), (5) Master Chemical Mechanism v2 (2000), (6) Bloss et al. (2005), (7) Tyndall et al. (2001), and (8) Ravishankara et al. (2002).

\begin{tabular}{|c|c|c|c|c|c|}
\hline Label & Reaction & $A$ & $\alpha$ & $\frac{E_{\mathrm{a}}}{R}$ & Ref. \\
\hline 1 & $\mathrm{HO}_{2}+\mathrm{NO} \rightarrow \mathrm{OH}+\mathrm{NO}_{2}$ & $3.60 \mathrm{E}-12$ & 0.00 & -270.0 & 2 \\
\hline 2 & $\mathrm{HO}_{2}+\mathrm{NO}_{3} \rightarrow \mathrm{OH}+\mathrm{NO}_{2}$ & $4.00 \mathrm{E}-12$ & 0.00 & 0.0 & 2 \\
\hline 3 & $\mathrm{HO}_{2}+\mathrm{O}_{3} \rightarrow \mathrm{OH}+\mathrm{O}_{2}$ & $2.03 \mathrm{E}-16$ & 4.57 & -693.0 & 2 \\
\hline 4 & $\mathrm{HO}_{2}+\mathrm{HO}_{2} \rightarrow \mathrm{H}_{2} \mathrm{O}_{2}$ & $2.20 \mathrm{E}-13$ & 0.00 & -600.0 & 2 \\
\hline 5 & $\mathrm{HO}_{2}+\mathrm{MeOO} \rightarrow \mathrm{MeOOH}$ & $3.80 \mathrm{E}-13$ & 0.00 & -780.0 & 4 \\
\hline 6 & $\mathrm{HO}_{2}+\mathrm{MeOO} \rightarrow \mathrm{HCHO}$ & $3.80 \mathrm{E}-13$ & 0.00 & -780.0 & 4 \\
\hline 7 & $\mathrm{HO}_{2}+\mathrm{EtOO} \rightarrow \mathrm{EtOOH}$ & $3.80 \mathrm{E}-13$ & 0.00 & -900.0 & 1 \\
\hline 8 & $\mathrm{HO}_{2}+\mathrm{MeCO}_{3} \rightarrow \mathrm{MeCO}_{3} \mathrm{H}$ & $2.08 \mathrm{E}-13$ & 0.00 & -980.0 & 4 \\
\hline 9 & $\mathrm{HO}_{2}+\mathrm{MeCO}_{3} \rightarrow \mathrm{MeCO}_{2} \mathrm{H}+\mathrm{O}_{3}$ & $1.04 \mathrm{E}-13$ & 0.00 & -980.0 & 4 \\
\hline 10 & $\mathrm{HO}_{2}+\mathrm{MeCO}_{3} \rightarrow \mathrm{OH}+\mathrm{MeOO}$ & $2.08 \mathrm{E}-13$ & 0.00 & -980.0 & 4 \\
\hline 11 & $\mathrm{HO}_{2}+n-\mathrm{PrOO} \rightarrow n-\mathrm{PrOOH}$ & $1.51 \mathrm{E}-13$ & 0.00 & -1300.0 & 5 \\
\hline 12 & $\mathrm{HO}_{2}+i-\mathrm{PrOO} \rightarrow i-\mathrm{PrOOH}$ & $1.51 \mathrm{E}-13$ & 0.00 & -1300.0 & 5 \\
\hline 13 & $\mathrm{HO}_{2}+\mathrm{EtCO}_{3} \rightarrow \mathrm{O}_{2}+\mathrm{EtCO}_{3} \mathrm{H}$ & $3.05 \mathrm{E}-13$ & 0.00 & -1040.0 & 5 \\
\hline 14 & $\mathrm{HO}_{2}+\mathrm{EtCO}_{3} \rightarrow \mathrm{O}_{3}+\mathrm{EtCO}_{2} \mathrm{H}$ & $1.25 \mathrm{E}-13$ & 0.00 & -1040.0 & 5 \\
\hline 15 & $\mathrm{HO}_{2}+\mathrm{MeCOCH} 2 \mathrm{OO} \rightarrow \mathrm{MeCOCH}_{2} \mathrm{OOH}$ & $1.36 \mathrm{E}-13$ & 0.00 & -1250.0 & 5 \\
\hline 16 & $\mathrm{MeOO}+\mathrm{NO} \rightarrow \mathrm{HO}_{2}+\mathrm{HCHO}+\mathrm{NO}_{2}$ & $2.95 \mathrm{E}-12$ & 0.00 & -285.0 & 2 \\
\hline 17 & $\mathrm{MeOO}+\mathrm{NO} \rightarrow \mathrm{MeONO}_{2}$ & $2.95 \mathrm{E}-15$ & 0.00 & -285.0 & 2 \\
\hline 18 & $\mathrm{MeOO}+\mathrm{NO}_{3} \rightarrow \mathrm{HO}_{2}+\mathrm{HCHO}+\mathrm{NO}_{2}$ & $1.30 \mathrm{E}-12$ & 0.00 & 0.0 & 2 \\
\hline 19 & $\mathrm{MeOO}+\mathrm{MeOO} \rightarrow \mathrm{MeOH}+\mathrm{HCHO}$ & $1.03 \mathrm{E}-13$ & 0.00 & -365.0 & 2 \\
\hline 20 & $\mathrm{MeOO}+\mathrm{MeOO} \rightarrow \mathrm{HO}_{2}+\mathrm{HO}_{2}+\mathrm{HCHO}+\mathrm{HCHO}$ & $1.03 \mathrm{E}-13$ & 0.00 & -365.0 & 2 \\
\hline 21 & $\mathrm{MeOO}+\mathrm{MeCO}_{3} \rightarrow \mathrm{HO}_{2}+\mathrm{HCHO}+\mathrm{MeOO}$ & $1.80 \mathrm{E}-12$ & 0.00 & -500.0 & 1 \\
\hline 22 & $\mathrm{MeOO}+\mathrm{MeCO}_{3} \rightarrow \mathrm{MeCO} 2 \mathrm{H}+\mathrm{HCHO}$ & $2.00 \mathrm{E}-13$ & 0.00 & -500.0 & 1 \\
\hline 23 & $\mathrm{EtOO}+\mathrm{NO} \rightarrow \mathrm{MeCHO}+\mathrm{HO}_{2}+\mathrm{NO}_{2}$ & $2.60 \mathrm{E}-12$ & 0.00 & -380.0 & 2 \\
\hline 24 & $\mathrm{EtOO}+\mathrm{NO}_{3} \rightarrow \mathrm{MeCHO}+\mathrm{HO}_{2}+\mathrm{NO}_{2}$ & $2.30 \mathrm{E}-12$ & 0.00 & 0.0 & 1 \\
\hline 25 & $\mathrm{EtOO}+\mathrm{MeCO}_{3} \rightarrow \mathrm{MeCHO}+\mathrm{HO}_{2}+\mathrm{MeOO}$ & $4.40 \mathrm{E}-13$ & 0.00 & -1070.0 & 2 \\
\hline 26 & $\mathrm{MeCO}_{3}+\mathrm{NO} \rightarrow \mathrm{MeOO}+\mathrm{CO}_{2}+\mathrm{NO}_{2}$ & $7.50 \mathrm{E}-12$ & 0.00 & -290.0 & 2 \\
\hline 27 & $\mathrm{MeCO}_{3}+\mathrm{NO}_{3} \rightarrow \mathrm{MeOO}+\mathrm{CO}_{2}+\mathrm{NO}_{2}$ & 4.00E-12 & 0.00 & 0.0 & 5 \\
\hline 28 & $n-\mathrm{PrOO}+\mathrm{NO} \rightarrow \mathrm{EtCHO}+\mathrm{HO}_{2}+\mathrm{NO}_{2}$ & $2.90 \mathrm{E}-12$ & 0.00 & -350.0 & 2 \\
\hline 29 & $n-\mathrm{PrOO}+\mathrm{NO}_{3} \rightarrow \mathrm{EtCHO}+\mathrm{HO}_{2}+\mathrm{NO}_{2}$ & $2.50 \mathrm{E}-12$ & 0.00 & 0.0 & 5 \\
\hline 30 & $i-\mathrm{PrOO}+\mathrm{NO} \rightarrow \mathrm{Me}_{2} \mathrm{CO}+\mathrm{HO}_{2}+\mathrm{NO}_{2}$ & $2.70 \mathrm{E}-12$ & 0.00 & -360.0 & 2 \\
\hline 31 & $i-\mathrm{PrOO}+\mathrm{NO}_{3} \rightarrow \mathrm{Me}_{2} \mathrm{CO}+\mathrm{HO}_{2}+\mathrm{NO}_{2}$ & $2.50 \mathrm{E}-12$ & 0.00 & 0.0 & 5 \\
\hline 32 & $\mathrm{EtCO}_{3}+\mathrm{NO} \rightarrow \mathrm{EtOO}+\mathrm{CO}_{2}+\mathrm{NO}_{2}$ & $6.70 \mathrm{E}-12$ & 0.00 & -340.0 & 2 \\
\hline 33 & $\mathrm{EtCO}_{3}+\mathrm{NO}_{3} \rightarrow \mathrm{EtOO}+\mathrm{CO}_{2}+\mathrm{NO}_{2}$ & $4.00 \mathrm{E}-12$ & 0.00 & 0.0 & 5 \\
\hline 34 & $\mathrm{MeCOCH}_{2} \mathrm{OO}+\mathrm{NO} \rightarrow \mathrm{MeCO}_{3}+\mathrm{HCHO}+\mathrm{NO}_{2}$ & $2.80 \mathrm{E}-12$ & 0.00 & -300.0 & 7 \\
\hline 35 & $\mathrm{MeCOCH} \mathrm{H}_{2} \mathrm{OO}+\mathrm{NO}_{3} \rightarrow \mathrm{MeCO}_{3}+\mathrm{HCHO}+\mathrm{NO}_{2}$ & $2.50 \mathrm{E}-12$ & 0.00 & 0.0 & 5 \\
\hline 36 & $\mathrm{NO}+\mathrm{NO}_{3} \rightarrow \mathrm{NO}_{2}+\mathrm{NO}_{2}$ & $1.80 \mathrm{E}-11$ & 0.00 & -110.0 & 2 \\
\hline 37 & $\mathrm{NO}+\mathrm{O}_{3} \rightarrow \mathrm{NO}_{2}$ & $1.40 \mathrm{E}-12$ & 0.00 & 1310.0 & 2 \\
\hline 38 & $\mathrm{NO}_{2}+\mathrm{O}_{3} \rightarrow \mathrm{NO}_{3}$ & $1.40 \mathrm{E}-13$ & 0.00 & 2470.0 & 1 \\
\hline 39 & $\mathrm{NO}_{3}+\mathrm{HCHO} \rightarrow \mathrm{HONO}_{2}+\mathrm{HO}_{2}+\mathrm{CO}$ & $2.00 \mathrm{E}-12$ & 0.00 & 2440.0 & 2 \\
\hline 40 & $\mathrm{NO}_{3}+\mathrm{MeCHO} \rightarrow \mathrm{HONO}_{2}+\mathrm{MeCO}_{3}$ & $1.40 \mathrm{E}-12$ & 0.00 & 1860.0 & 2 \\
\hline 41 & $\mathrm{NO}_{3}+\mathrm{EtCHO} \rightarrow \mathrm{HONO}_{2}+\mathrm{EtCO}_{3}$ & $3.46 \mathrm{E}-12$ & 0.00 & 1862.0 & 6 \\
\hline 42 & $\mathrm{NO}_{3}+\mathrm{Me}_{2} \mathrm{CO} \rightarrow \mathrm{HONO}_{2}+\mathrm{MeCOCH}_{2} \mathrm{OO}$ & $3.00 \mathrm{E}-17$ & 0.00 & 0.0 & 2 \\
\hline 43 & $\mathrm{~N}_{2} \mathrm{O}_{5}+\mathrm{H}_{2} \mathrm{O} \rightarrow \mathrm{HONO}_{2}+\mathrm{HONO}_{2}$ & $2.50 \mathrm{E}-22$ & 0.00 & 0.0 & 2 \\
\hline 44 & $\mathrm{O}(3 \mathrm{P})+\mathrm{O}_{3} \rightarrow \mathrm{O}_{2}+\mathrm{O}_{2}$ & $8.00 \mathrm{E}-12$ & 0.00 & 2060.0 & 1 \\
\hline 45 & $\mathrm{O}(1 \mathrm{D})+\mathrm{CH}_{4} \rightarrow \mathrm{OH}+\mathrm{MeOO}$ & $1.05 \mathrm{E}-10$ & 0.00 & 0.0 & 2 \\
\hline 46 & $\mathrm{O}(1 \mathrm{D})+\mathrm{CH}_{4} \rightarrow \mathrm{HCHO}+\mathrm{H}_{2}$ & $7.50 \mathrm{E}-12$ & 0.00 & 0.0 & 2 \\
\hline 47 & $\mathrm{O}(1 \mathrm{D})+\mathrm{CH}_{4} \rightarrow \mathrm{HCHO}+\mathrm{HO}_{2}+\mathrm{HO}_{2}$ & $3.45 \mathrm{E}-11$ & 0.00 & 0.0 & 2 \\
\hline 48 & $\mathrm{O}(1 \mathrm{D})+\mathrm{H}_{2} \mathrm{O} \rightarrow \mathrm{OH}+\mathrm{OH}$ & $2.20 \mathrm{E}-10$ & 0.00 & 0.0 & 1 \\
\hline
\end{tabular}


Table 5. Continued.

\begin{tabular}{|c|c|c|c|c|c|}
\hline Label & Reaction & $A$ & $\alpha$ & $\frac{E_{\mathrm{a}}}{R}$ & Ref. \\
\hline 49 & $\mathrm{O}(1 \mathrm{D})+\mathrm{N}_{2} \rightarrow \mathrm{O}(3 \mathrm{P})+\mathrm{N}_{2}$ & $2.10 \mathrm{E}-11$ & 0.00 & -115.0 & 8 \\
\hline 50 & $\mathrm{O}(1 \mathrm{D})+\mathrm{O}_{2} \rightarrow \mathrm{O}(3 \mathrm{P})+\mathrm{O}_{2}$ & $3.20 \mathrm{E}-11$ & 0.00 & -67.0 & 1 \\
\hline 51 & $\mathrm{OH}+\mathrm{CH}_{4} \rightarrow \mathrm{H}_{2} \mathrm{O}+\mathrm{MeOO}$ & $1.85 \mathrm{E}-12$ & 0.00 & 1690.0 & 2 \\
\hline 52 & $\mathrm{OH}+\mathrm{C}_{2} \mathrm{H}_{6} \rightarrow \overline{\mathrm{H}}_{2} \mathrm{O}+\mathrm{EtOO}$ & $6.90 \mathrm{E}-12$ & 0.00 & 1000.0 & 2 \\
\hline 53 & $\mathrm{OH}+\mathrm{C}_{3} \mathrm{H}_{8} \rightarrow n-\mathrm{PrOO}+\mathrm{H}_{2} \mathrm{O}$ & $7.60 \mathrm{E}-12$ & 0.00 & 585.0 & 2 \\
\hline 54 & $\mathrm{OH}+\mathrm{C}_{3} \mathrm{H}_{8} \rightarrow i-\mathrm{PrOO}+\mathrm{H}_{2} \mathrm{O}$ & $7.60 \mathrm{E}-12$ & 0.00 & 585.0 & 2 \\
\hline 55 & $\mathrm{OH}+\mathrm{CO} \rightarrow \mathrm{HO}_{2}$ & $1.44 \mathrm{E}-13$ & 0.00 & 0.0 & 4 \\
\hline 56 & $\mathrm{OH}+\mathrm{EtCHO} \rightarrow \mathrm{H}_{2} \mathrm{O}+\mathrm{EtCO}_{3}$ & $5.10 \mathrm{E}-12$ & 0.00 & -405.0 & 2 \\
\hline 57 & $\mathrm{OH}+\mathrm{EtOOH} \rightarrow \mathrm{H}_{2} \mathrm{O}+\mathrm{MeCHO}+\mathrm{OH}$ & 8.01E-12 & 0.00 & 0.0 & 6 \\
\hline 58 & $\mathrm{OH}+\mathrm{EtOOH} \rightarrow \mathrm{H}_{2} \mathrm{O}+\mathrm{EtOO}$ & $1.90 \mathrm{E}-12$ & 0.00 & -190.0 & 5 \\
\hline 59 & $\mathrm{OH}+\mathrm{H}_{2} \rightarrow \mathrm{H}_{2} \mathrm{O}+\mathrm{HO}_{2}$ & $7.70 \mathrm{E}-12$ & 0.00 & 2100.0 & 2 \\
\hline 60 & $\mathrm{OH}+\mathrm{H}_{2} \mathrm{O}_{2} \rightarrow \mathrm{H}_{2} \mathrm{O}+\mathrm{HO}_{2}$ & $2.90 \mathrm{E}-12$ & 0.00 & 160.0 & 1 \\
\hline 61 & $\mathrm{OH}+\mathrm{HCHO} \rightarrow \mathrm{H}_{2} \mathrm{O}+\mathrm{HO}_{2}+\mathrm{CO}$ & $5.40 \mathrm{E}-12$ & 0.00 & -135.0 & 3 \\
\hline 62 & $\mathrm{OH}+\mathrm{HO}_{2} \rightarrow \mathrm{H}_{2} \mathrm{O}$ & $4.80 \mathrm{E}-11$ & 0.00 & -250.0 & 1 \\
\hline 63 & $\mathrm{OH}+\mathrm{HO}_{2} \mathrm{NO}_{2} \rightarrow \mathrm{H}_{2} \mathrm{O}+\mathrm{NO}_{2}$ & $1.90 \mathrm{E}-12$ & 0.00 & -270.0 & 2 \\
\hline 64 & $\mathrm{OH}+\mathrm{HONO}_{2} \rightarrow \mathrm{H}_{2} \mathrm{O}+\mathrm{NO}_{3}$ & $1.50 \mathrm{E}-13$ & 0.00 & 0.0 & 2 \\
\hline 65 & $\mathrm{OH}+\mathrm{HONO} \rightarrow \mathrm{H}_{2} \mathrm{O}+\mathrm{NO}_{2}$ & $2.50 \mathrm{E}-12$ & 0.00 & -260.0 & 2 \\
\hline 66 & $\mathrm{OH}+\mathrm{MeOOH} \rightarrow \mathrm{H}_{2} \mathrm{O}+\mathrm{HCHO}+\mathrm{OH}$ & $1.02 \mathrm{E}-12$ & 0.00 & -190.0 & 2 \\
\hline 67 & $\mathrm{OH}+\mathrm{MeOOH} \rightarrow \mathrm{H}_{2} \mathrm{O}+\mathrm{MeOO}$ & $1.89 \mathrm{E}-12$ & 0.00 & -190.0 & 2 \\
\hline 68 & $\mathrm{OH}+\mathrm{MeONO}_{2} \rightarrow \mathrm{HCHO}+\mathrm{NO}_{2}+\mathrm{H}_{2} \mathrm{O}$ & $4.00 \mathrm{E}-13$ & 0.00 & 845.0 & 1 \\
\hline 69 & $\mathrm{OH}+\mathrm{Me}_{2} \mathrm{CO} \rightarrow \mathrm{H}_{2} \mathrm{O}+\mathrm{MeCOCH}_{2} \mathrm{OO}$ & $8.80 \mathrm{E}-12$ & 0.00 & 1320.0 & 2 \\
\hline 70 & $\mathrm{OH}+\mathrm{Me}_{2} \mathrm{CO} \rightarrow \mathrm{H}_{2} \mathrm{O}+\mathrm{MeCOCH}_{2} \mathrm{OO}$ & $1.70 \mathrm{E}-14$ & 0.00 & -420.0 & 2 \\
\hline 71 & $\mathrm{OH}+\mathrm{MeCOCH}_{2} \mathrm{OOH} \rightarrow \mathrm{H}_{2} \mathrm{O}+\mathrm{MeCOCH}_{2} \mathrm{OO}$ & $1.90 \mathrm{E}-12$ & 0.00 & -190.0 & 6 \\
\hline 72 & $\mathrm{OH}+\mathrm{MeCOCH}_{2} \mathrm{OOH} \rightarrow \mathrm{OH}+\mathrm{MGLY}$ & 8.39E-12 & 0.00 & 0.0 & 6 \\
\hline 73 & $\mathrm{OH}+\mathrm{MeCHO} \rightarrow \mathrm{H}_{2} \mathrm{O}+\mathrm{MeCO}_{3}$ & 4.40E-12 & 0.00 & -365.0 & 6 \\
\hline 74 & $\mathrm{OH}+\mathrm{NO}_{3} \rightarrow \mathrm{HO}_{2}+\mathrm{NO}_{2}$ & $2.00 \mathrm{E}-11$ & 0.00 & 0.0 & 1 \\
\hline 75 & $\mathrm{OH}+\mathrm{O}_{3} \rightarrow \mathrm{HO}_{2}+\mathrm{O}_{2}$ & $1.70 \mathrm{E}-12$ & 0.00 & 940.0 & 2 \\
\hline 76 & $\mathrm{OH}+\mathrm{OH} \rightarrow \mathrm{H}_{2} \mathrm{O}+\mathrm{O}(3 \mathrm{P})$ & $6.31 \mathrm{E}-14$ & 2.60 & -945.0 & 2 \\
\hline 77 & $\mathrm{OH}+\mathrm{PAN} \rightarrow \mathrm{HCHO}+\mathrm{NO}_{2}+\mathrm{H}_{2} \mathrm{O}$ & $3.00 \mathrm{E}-14$ & 0.00 & 0.0 & 1 \\
\hline 78 & $\mathrm{OH}+\mathrm{PPAN} \rightarrow \mathrm{MeCHO}+\mathrm{NO}_{2}+\mathrm{H}_{2} \mathrm{O}$ & $1.27 \mathrm{E}-12$ & 0.00 & 0.0 & 5 \\
\hline 79 & $\mathrm{OH}+n-\mathrm{PrOOH} \rightarrow n-\mathrm{PrOO}+\mathrm{H}_{2} \mathrm{O}$ & $1.90 \mathrm{E}-12$ & 0.00 & -190.0 & 5 \\
\hline 80 & $\mathrm{OH}+n-\mathrm{PrOOH} \rightarrow \mathrm{EtCHO}+\mathrm{H}_{2} \mathrm{O}+\mathrm{OH}$ & $1.10 \mathrm{E}-11$ & 0.00 & 0.0 & 6 \\
\hline 81 & $\mathrm{OH}+i-\mathrm{PrOOH} \rightarrow i-\mathrm{PrOO}+\mathrm{H}_{2} \mathrm{O}$ & $1.90 \mathrm{E}-12$ & 0.00 & -190.0 & 5 \\
\hline 82 & $\mathrm{OH}+i-\mathrm{PrOOH} \rightarrow \mathrm{Me}_{2} \mathrm{CO}+\mathrm{OH}$ & $1.66 \mathrm{E}-11$ & 0.00 & 0.0 & 6 \\
\hline 83 & $\mathrm{O}(3 \mathrm{P})+\mathrm{NO}_{2} \rightarrow \mathrm{NO}+\mathrm{O}_{2}$ & $5.50 \mathrm{E}-12$ & 0.00 & -188.0 & 2 \\
\hline
\end{tabular}

\subsection{Photolysis}

The tropospheric chemistry schemes in UKCA can make use of three options in relation to photolysis. In the simplest setup, photolysis rates are calculated offline in the Cambridge 2-D model (Law and Pyle, 1993) using the two-stream approach of Hough (1988). They are read in by UKCA on the first timestep of the model integration and interpolated in time and space at each model grid box. Although this treatment gives realistic diurnal and seasonal variations in photolysis rates, there is no variability associated with cloud cover, surface albedo and/or aerosols; the 2-D rates were calculated with a climatological cloud cover and prescribed surface albedo and aerosol loading. One alternative option available in UKCA is the use of Fast-J (Wild et al., 2000), which provides a flexible and accurate scheme for the online calculation of photolysis rates in the presence of both cloud and aerosol layers. It solves the full radiative transfer equations with a reduced expansion of the scattering phase function over a relatively small number of wavelength bins. As well as assessing the accuracy of the calculated photolysis rates from Fast-J relative to clear-sky photolysis rates, Wild et al. (2000) also noted that the upper troposphere is a key region for climate forcing by $\mathrm{O}_{3}$ and hence, the accurate treatment of photolysis in this region of the atmosphere is critical in chemistry-climate models.

Figure 11 shows the zonal mean photolysis rate for the $\mathrm{NO}_{2} \rightarrow \mathrm{NO}+\mathrm{O}(3 \mathrm{P})$ reaction for January and July from both the offline 2-D photolysis scheme and Fast-J. Also included are scatter plots comparing the two. Both schemes capture the seasonal, latitudinal, and altitude dependence of $J\left(\mathrm{NO}_{2}\right)$. The figure also clearly shows that although there are differences in photolysis rates from the two schemes due to differences in formulation, cloud and aerosol distributions, they 
Table 6. Uni- and termolecular reactions in the UKCA standard tropospheric (StdTrop) scheme, where $\mathrm{Me}_{2}=\mathrm{CH}_{3}, \mathrm{Et}_{\mathrm{t}}=\mathrm{C}_{2} \mathrm{H}_{5}, \mathrm{PAN}=\mathrm{peroxy}$ acetyl nitrate, and PPAN = peroxy propionyl nitrate. Rate coefficients for termolecular reactions are calculated using a modified LindemannHinshelwood mechanism, i.e. $k=\left(\frac{k_{\mathrm{o}}[M]}{1+\frac{k_{\mathrm{o}}[M]}{k_{\infty}}}\right) F_{\mathrm{c}}^{1+\log \left[\frac{k_{\mathrm{o}}[M]}{k_{\infty}}\right]^{2}}$, where $k_{\mathrm{O}}$ and $k_{\infty}$ are the low- and high-pressure limits calculated in the same manner as bimolecular rate coefficients, and $[M]$ is the number density in molecules $\mathrm{cm}^{-3}$. References: (1) Atkinson et al. (2000), (2) Atkinson et al. (2003).

\begin{tabular}{|c|c|c|c|c|c|c|c|c|c|}
\hline \multirow{2}{*}{ Label } & \multirow{2}{*}{ Reaction } & \multirow{2}{*}{$F_{\mathrm{c}}$} & \multicolumn{3}{|c|}{ Low-pressure limit, $k_{\mathrm{O}}$} & \multicolumn{3}{|c|}{ High-pressure limit, $k_{\infty}$} & \multirow{2}{*}{ Ref. } \\
\hline & & & A & $\alpha$ & $\frac{E_{\mathrm{a}}}{R}$ & A & $\alpha$ & $\frac{E_{\mathrm{a}}}{R}$ & \\
\hline 1 & $\mathrm{HO}_{2}+\mathrm{HO}_{2}+\mathrm{M} \rightarrow \mathrm{H}_{2} \mathrm{O}_{2}+\mathrm{O}_{2}+\mathrm{M}$ & 0.00 & $1.90 \mathrm{E}-33$ & 0.00 & -980.0 & $0.00 \mathrm{E}+00$ & 0.00 & 0.0 & 1 \\
\hline 2 & $\mathrm{HO}_{2}+\mathrm{NO}_{2}+\mathrm{M} \rightarrow \mathrm{HO}_{2} \mathrm{NO}_{2}+\mathrm{M}$ & 0.60 & $1.80 \mathrm{E}-31$ & -3.20 & 0.0 & $4.70 \mathrm{E}-12$ & 0.00 & 0.0 & 1 \\
\hline 3 & $\mathrm{HO}_{2} \mathrm{NO}_{2}+\mathrm{M} \rightarrow \mathrm{HO}_{2}+\mathrm{NO}_{2}+\mathrm{M}$ & 0.60 & 4.10E-05 & 0.00 & 10650.0 & $4.80 \mathrm{E}+15$ & 0.00 & 11170.0 & 2 \\
\hline 4 & $\mathrm{MeCO}_{3}+\mathrm{NO}_{2}+\mathrm{M} \rightarrow \mathrm{PAN}+\mathrm{M}$ & 0.30 & $2.70 \mathrm{E}-28$ & -7.10 & 0.0 & $1.20 \mathrm{E}-11$ & -0.90 & 0.0 & 1 \\
\hline 5 & $\mathrm{PAN}+\mathrm{M} \rightarrow \mathrm{MeCO}_{3}+\mathrm{NO}_{2}+\mathrm{M}$ & 0.30 & 4.90E-03 & 0.00 & 12100.0 & $5.40 \mathrm{E}+16$ & 0.00 & 13830.0 & 1 \\
\hline 6 & $\mathrm{~N}_{2} \mathrm{O}_{5}+\mathrm{M} \rightarrow \mathrm{NO}_{2}+\mathrm{NO}_{3}+\mathrm{M}$ & 0.35 & $1.30 \mathrm{E}-03$ & -3.50 & 11000.0 & $9.70 \mathrm{E}+14$ & 0.10 & 11080.0 & 2 \\
\hline 7 & $\mathrm{NO}_{2}+\mathrm{NO}_{3}+\mathrm{M} \rightarrow \mathrm{N}_{2} \mathrm{O}_{5}+\mathrm{M}$ & 0.35 & $3.60 \mathrm{E}-30$ & -4.10 & 0.0 & $1.90 \mathrm{E}-12$ & 0.20 & 0.0 & 2 \\
\hline 8 & $\mathrm{O}(3 \mathrm{P})+\mathrm{O}_{2}+\mathrm{M} \rightarrow \mathrm{O}_{3}+\mathrm{M}$ & 0.00 & $5.70 \mathrm{E}-34$ & -2.60 & 0.0 & $0.00 \mathrm{E}+00$ & 0.00 & 0.0 & 2 \\
\hline 9 & $\mathrm{OH}+\mathrm{NO}+\mathrm{M} \rightarrow \mathrm{HONO}+\mathrm{M}$ & 1420.00 & $7.40 \mathrm{E}-31$ & -2.40 & 0.0 & $3.30 \mathrm{E}-11$ & -0.30 & 0.0 & 2 \\
\hline 10 & $\mathrm{OH}+\mathrm{NO}_{2}+\mathrm{M} \rightarrow \mathrm{HONO}_{2}+\mathrm{M}$ & 0.40 & $3.30 \mathrm{E}-30$ & -3.00 & 0.0 & $4.10 \mathrm{E}-11$ & 0.00 & 0.0 & 2 \\
\hline 11 & $\mathrm{OH}+\mathrm{OH}+\mathrm{M} \rightarrow \mathrm{H}_{2} \mathrm{O}_{2}+\mathrm{M}$ & 0.50 & $6.90 \mathrm{E}-31$ & -0.80 & 0.0 & $2.60 \mathrm{E}-11$ & 0.00 & 0.0 & 1 \\
\hline 12 & $\mathrm{EtCO}_{3}+\mathrm{NO}_{2}+\mathrm{M} \rightarrow \mathrm{PPAN}+\mathrm{M}$ & 0.30 & $2.70 \mathrm{E}-28$ & -7.10 & 0.0 & $1.20 \mathrm{E}-11$ & -0.90 & 0.0 & 2 \\
\hline 13 & $\mathrm{PPAN}+\mathrm{M} \rightarrow \mathrm{EtCO}_{3}+\mathrm{NO}_{2}+\mathrm{M}$ & 0.30 & $1.70 \mathrm{E}-03$ & 0.00 & 12100.0 & $5.40 \mathrm{E}+16$ & 0.00 & 13830.0 & 1 \\
\hline
\end{tabular}
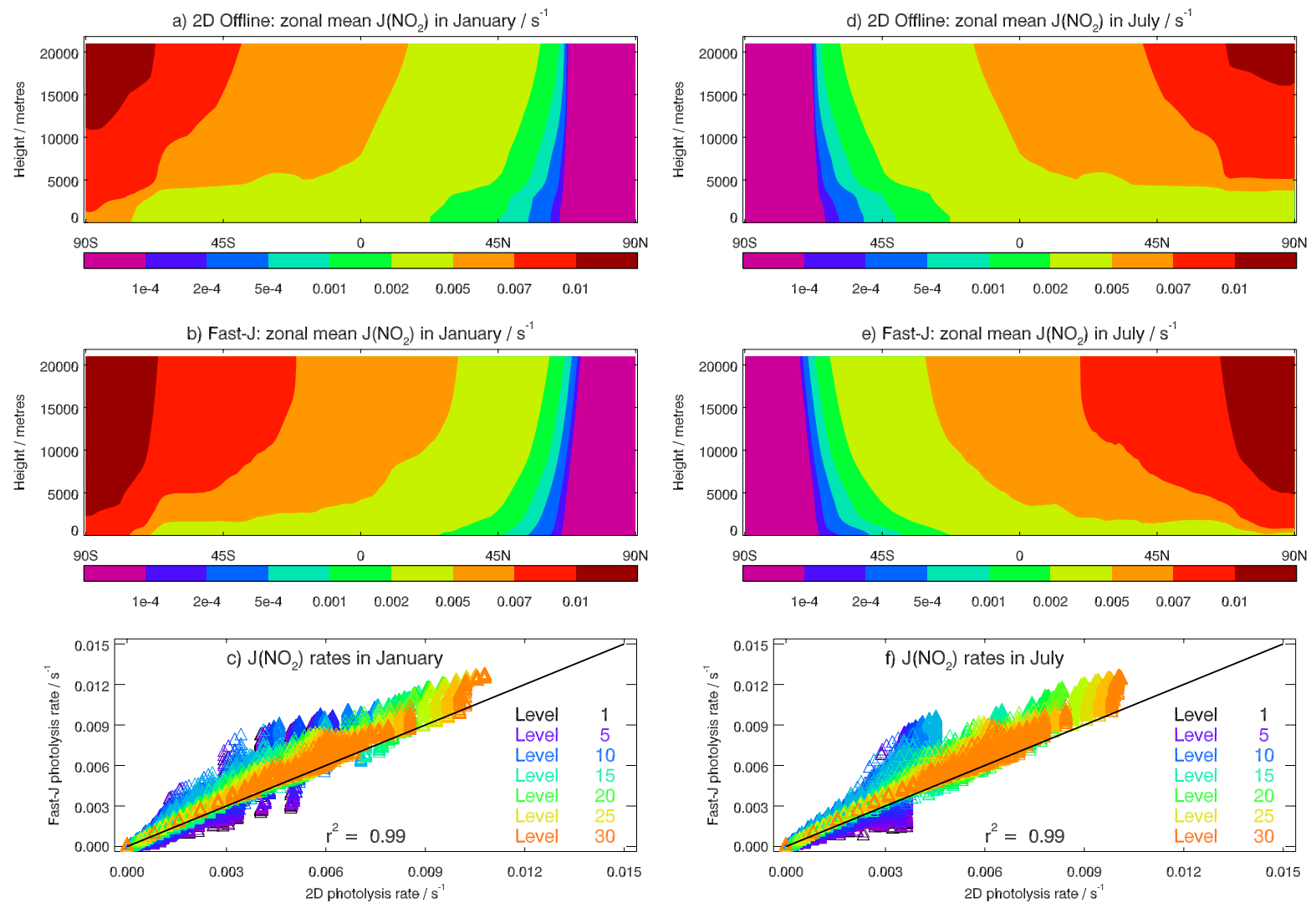

Fig. 11. Comparison between zonal mean $J\left(\mathrm{NO}_{2}\right)$ rates in January and in July from the offline 2-D photolysis scheme in (a) and (d) and from the online Fast-J scheme in (b) and (e). Scatter plots of the $J\left(\mathrm{NO}_{2}\right)$ photolysis rate from both schemes are shown in (c) and (f). 
Table 7. Additional tracers/species associated with the Mainz Isoprene Mechanism in the UKCA TropIsop chemistry scheme, where $\mathrm{Me}=\mathrm{CH}_{3}, \mathrm{IS}=\mathrm{C}_{5} \mathrm{H}_{7}$, ISON = lumped peroxy alkyl nitrates (from $\mathrm{C}_{5} \mathrm{H}_{8}+\mathrm{NO}_{3}$ ) and hydroxy alkyl nitrates (from $\mathrm{ISO}_{2}+\mathrm{NO}$ ), MACR = lumped species consisting of methacrolein, methyl vinyl ketone and other $\mathrm{C}_{4}$ carbonyls from isoprene oxidation, MPAN = peroxy methacrylic nitric anhydride (and other higher PANs from isoprene chemistry), HACET = hydroxyacetone and other $\mathrm{C}_{3}$ ketones, MGLY = methylglyoxal and other $\mathrm{C}_{3}$ carbonyls, and NALD = nitrooxy acetaldehyde.

\begin{tabular}{lc}
\hline Species Name & Treatment \\
\hline $\mathrm{ISO}_{2}$ & Steady State \\
$\mathrm{MACRO}_{2}$ & Steady State \\
$\mathrm{C}_{5} \mathrm{H}_{8}$ & Transported Tracer \\
ISOOH & Transported Tracer \\
ISON & Transported Tracer \\
MACR & Transported Tracer \\
MACROOH & Transported Tracer \\
MPAN & Transported Tracer \\
HACET & Transported Tracer \\
MGLY & Transported Tracer \\
NALD & Transported Tracer \\
HCOOH & Transported Tracer \\
$\mathrm{MeCO}_{3} \mathrm{H}$ & Transported Tracer \\
$\mathrm{MeCO}_{2} \mathrm{H}$ & Transported Tracer \\
\hline
\end{tabular}

are within the spread of photolysis rates from a recent intercomparison of chemistry-climate models (Eyring et al., 2010a). Offline photolysis has been used within UKCA in all the Met Office Hadley Centre's centennial integrations for Phase 5 of the Coupled Model Intercomparison Project (CMIP5) and the Atmospheric Chemistry and Climate Model Intercomparison Project (ACCMIP) simulations using the Earth System version of the HadGEM2 model (Collins et al., 2011; Jones et al., 2011). It is also used in the main model integration being evaluated here. However, a sensitivity experiment in which Fast-J was used in place of the offline photolysis scheme was carried out and is discussed in Section 6. Fast-J is also used routinely in the context of air quality forecasts from UKCA which are discussed in Savage et al. (2013).

More recently, there has been work to add Fast-JX (Neu et al., 2007) to UKCA. This is an improved version of the original Fast-J scheme, with scattering calculations extended up to the stratosphere and lower mesosphere as well as updates to the treatment of optically thick clouds and to the solar flux used. In particular, the switch from Fast-J to FastJX increases all photolysis cross sections, with the increase being larger at lower wavelengths. Rates, such as $J\left(\mathrm{O}^{1} \mathrm{D}\right)$, are more dramatically affected. The implementation and use of Fast-JX in comparison with the offline photolysis scheme is evaluated in Telford et al. (2013), in which discrepancies in $J\left(\mathrm{O}^{1} \mathrm{D}\right)$ between the offline scheme and Fast-JX are discussed.
Table 8. Additional photolytic reactions associated with the Mainz Isoprene Mechanism in the UKCA TropIsop chemistry scheme, where IS $=\mathrm{C}_{5} \mathrm{H}_{7}$, ISON = lumped peroxy alkyl nitrates (from $\mathrm{C}_{5} \mathrm{H}_{8}+\mathrm{NO}_{3}$ ) and hydroxy alkyl nitrates (from $\mathrm{ISO}_{2}+\mathrm{NO}$ ), MACR = lumped species consisting of methacrolein, methyl vinyl ketone and other $\mathrm{C}_{4}$ carbonyls from isoprene chemistry, MPAN = peroxy methacrylic nitric anhydride (and other higher PANs from isoprene chemistry), HACET $=$ hydroxyacetone and other $\mathrm{C}_{3}$ ketones, MGLY $=$ methylglyoxal nd other $\mathrm{C}_{3}$ carbonyls, and $\mathrm{NALD}=$ nitrooxy acetaldehyde.

\begin{tabular}{cl}
\hline Label & Reaction \\
\hline 26 & ISOOH $+h v \rightarrow \mathrm{OH}+\mathrm{MACR}+\mathrm{HCHO}+\mathrm{HO}_{2}$ \\
27 & $\mathrm{ISON}+h v \rightarrow \mathrm{NO}_{2}+\mathrm{MACR}+\mathrm{HCHO}+\mathrm{HO}_{2}$ \\
28 & $\mathrm{MACR}+h v \rightarrow \mathrm{MeCO}_{3}+\mathrm{HCHO}+\mathrm{CO}+\mathrm{HO}_{2}$ \\
29 & $\mathrm{MPAN}+h v \rightarrow \mathrm{MACRO}_{2}+\mathrm{NO}_{2}$ \\
30 & $\mathrm{MACROOH}+h v \rightarrow \mathrm{OH}+\mathrm{OH}+\mathrm{HO}_{2}+\mathrm{HO}_{2}$ \\
31 & $\mathrm{MACROOH}+h v \rightarrow \mathrm{HACET}+\mathrm{CO}+\mathrm{MGLY}+\mathrm{HCHO}_{32} \mathrm{HACET}+h v \rightarrow \mathrm{MeCO}_{3}+\mathrm{HCHO}+\mathrm{HO}_{2}$ \\
33 & $\mathrm{MGLY}+h v \rightarrow \mathrm{MeCO}_{3}+\mathrm{CO}+\mathrm{HO}_{2}$ \\
34 & $\mathrm{NALD}+h v \rightarrow \mathrm{HCHO}+\mathrm{CO}+\mathrm{NO}_{2}+\mathrm{HO}_{2}$ \\
35 & $\mathrm{MeCO}_{3} \mathrm{H}+h v \rightarrow \mathrm{MeOO}+\mathrm{OH}$ \\
\hline
\end{tabular}

\subsection{Emissions}

The land-based anthropogenic, biomass burning, and shipping emissions used in the HadGEM2-UKCA coupled simulations being evaluated here make use of year-2000 emissions supplied as part of a new data set of gridded emissions covering the historical period 1850-2000 (Lamarque et al., 2010). A brief summary of the emissions will be given here. However, for a more complete discussion on the full set of aerosol and $\mathrm{O}_{3}$ precursor emissions and their application over the historical period in two composition-climate models, the reader is referred to Lamarque et al. (2010).

Land-based anthropogenic emissions take account of the following sectors: energy production and distribution, industry, land transport, residential and commercial sources, solvent use, agriculture, agricultural waste burning, waste and waste distribution, and were based on a combination of regional (Vestreng et al. (2006) inventory for Europe) and global inventories (e.g. EDGARv32 FT2000 data set from van Aardenne et al., 2005). Biomass burning emissions consider fires in forests, savanna, and grasslands. They are taken as the mean emissions from the 19972006 period from version 2 of the Global Fire Emissions Database (GFEDv2) inventory (van der Werf et al., 2006). Emissions from maritime transport are also considered. Fleet information and fuel consumption are taken from Eyring et al. (2010b) and non- $\mathrm{CO}_{2}$ emission totals for the year 2000 are taken as the mean of previous studies (Corbett and Koehler, 2003; Eyring et al., 2005; Endresen et al., 2003, 2007). They are then distributed globally and monthly using the International Comprehensive OceanAtmosphere Data Set (ICOADS; Wang et al., 2008). 
Table 9. Additional bimolecular reactions associated with the Mainz Isoprene Mechanism in the UKCA TropIsop chemistry scheme, where IS $=\mathrm{C}_{5} \mathrm{H}_{7}$, ISON = lumped peroxy alkyl nitrates (from $\mathrm{C}_{5} \mathrm{H}_{8}+\mathrm{NO}_{3}$ ) and hydroxy alkyl nitrates (from ISO $2+\mathrm{NO}$ ), MACR $=$ lumped species consisting of methacrolein, methyl vinyl ketone and other $\mathrm{C}_{4}$ carbonyls from isoprene chemistry, MPAN $=$ peroxy methacrylic nitric anhydride (and other higher PANs from isoprene chemistry), HACET = hydroxyacetone, MGLY = methylglyoxal, and NALD = nitrooxy acetaldehyde. The rate coefficient of a bimolecular reaction is given by $k=A\left(\frac{T}{300}\right)^{\alpha} \exp \left(\frac{-E_{\mathrm{a}}}{R T}\right)$, where $A$ is the pre-exponential factor, $T$ is the temperature, $R$ is the gas constant, $\alpha$ is the exponent for the $\left(\frac{T}{300}\right)$ temperature dependence, and $E_{\mathrm{a}}$ is the Arrhenius activation energy. References: (1) Atkinson et al. (2005), (2) Saunders et al. (2003), (3) Pöschl et al. (2000), and references therein.

\begin{tabular}{|c|c|c|c|c|c|}
\hline Label & Reaction & A & $\alpha$ & $\frac{E_{\mathrm{a}}}{R}$ & Ref. \\
\hline 84 & $\mathrm{OH}+\mathrm{C}_{5} \mathrm{H}_{8} \rightarrow \mathrm{ISO}_{2}$ & $2.70 \mathrm{E}-11$ & 0.00 & -390.0 & 1 \\
\hline 85 & $\mathrm{O}_{3}+\mathrm{C}_{5} \mathrm{H}_{8} \rightarrow 1.95 \mathrm{MACR}+1.74 \mathrm{HCHO}+0.3 \mathrm{MACRO}_{2}+0.3 \mathrm{MeCO}_{3}$ & $3.47 \mathrm{E}-15$ & 0.00 & 1995.0 & 1 \\
\hline 86 & $\mathrm{O}_{3}+\mathrm{C}_{5} \mathrm{H}_{8} \rightarrow 0.24 \mathrm{MeOO}+0.84 \mathrm{HCOOH}+0.42 \mathrm{CO}+0.27 \mathrm{H}_{2} \mathrm{O}_{2}$ & $3.47 \mathrm{E}-15$ & 0.00 & 1995.0 & 1 \\
\hline 87 & $\mathrm{O}_{3}+\mathrm{C}_{5} \mathrm{H}_{8} \rightarrow 0.75 \mathrm{HO}_{2}+0.75 \mathrm{OH}$ & $3.47 \mathrm{E}-15$ & 0.00 & 1995.0 & 1 \\
\hline 88 & $\mathrm{NO}_{3}+\mathrm{C}_{5} \mathrm{H}_{8} \rightarrow$ ISON & $3.15 \mathrm{e}-12$ & 0.0 & 450.0 & 1 \\
\hline 89 & $\mathrm{NO}+\mathrm{ISO}_{2} \rightarrow \mathrm{NO}_{2}+\mathrm{MACR}+\mathrm{HCHO}+\mathrm{HO}_{2}$ & $2.43 \mathrm{E}-12$ & 0.00 & -360.0 & 2 \\
\hline 90 & $\mathrm{NO}+\mathrm{ISO}_{2} \rightarrow \mathrm{ISON}$ & $1.12 \mathrm{E}-13$ & 0.00 & -360.0 & 3 \\
\hline 91 & $\mathrm{HO}_{2}+\mathrm{ISO}_{2} \rightarrow \mathrm{ISOOH}$ & $2.05 \mathrm{E}-13$ & 0.00 & -1300.0 & 3 \\
\hline 92 & $\mathrm{ISO}_{2}+\mathrm{ISO}_{2} \rightarrow 2 \mathrm{MACR}+\mathrm{HCHO}+\mathrm{HO}_{2}$ & $2.00 \mathrm{E}-12$ & 0.00 & 0.0 & 3 \\
\hline 93 & $\mathrm{OH}+\mathrm{ISOOH} \rightarrow \mathrm{MACR}+\mathrm{OH}$ & $1.00 \mathrm{E}-10$ & 0.00 & 0.0 & 3 \\
\hline 94 & $\mathrm{OH}+\mathrm{ISON} \rightarrow \mathrm{HACET}+\mathrm{NALD}$ & $1.30 \mathrm{E}-11$ & 0.00 & 0.0 & 3 \\
\hline 95 & $\mathrm{OH}+\mathrm{MACR} \rightarrow \mathrm{MACRO}_{2}$ & $1.30 \mathrm{E}-12$ & 0.00 & -610.0 & 1 \\
\hline 96 & $\mathrm{OH}+\mathrm{MACR} \rightarrow \mathrm{MACRO}_{2}$ & $4.00 \mathrm{E}-12$ & 0.00 & -380.0 & 1 \\
\hline 97 & $\mathrm{O}_{3}+\mathrm{MACR} \rightarrow 1.80 \mathrm{MGLY}+0.90 \mathrm{HCOOH}+0.64 \mathrm{HO}_{2}+0.44 \mathrm{CO}$ & $2.13 \mathrm{E}-16$ & 0.00 & 1520.0 & 1 \\
\hline 98 & $\mathrm{O}_{3}+\mathrm{MACR} \rightarrow 0.38 \mathrm{OH}+0.20 \mathrm{MeCO}_{3}$ & $2.13 \mathrm{E}-16$ & 0.00 & 1520.0 & 1 \\
\hline 99 & $\mathrm{O}_{3}+\mathrm{MACR} \rightarrow 1.80 \mathrm{MGLY}+0.90 \mathrm{HCOOH}+0.64 \mathrm{HO}_{2}+0.44 \mathrm{CO}$ & $3.50 \mathrm{E}-16$ & 0.00 & 2100.0 & 1 \\
\hline 100 & $\mathrm{O}_{3}+\mathrm{MACR} \rightarrow 0.38 \mathrm{OH}+0.20 \mathrm{MeCO}_{3}$ & $3.50 \mathrm{E}-16$ & 0.00 & 2100.0 & 1 \\
\hline 101 & $\mathrm{NO}+\mathrm{MACRO}_{2} \rightarrow 2.0 \mathrm{NO}_{2}+0.5 \mathrm{MeCO}_{3}+0.5 \mathrm{HACET}+0.5 \mathrm{CO}$ & $1.27 \mathrm{E}-12$ & 0.00 & -360.0 & 3 \\
\hline 102 & $\mathrm{NO}+\mathrm{MACRO}_{2} \rightarrow 1.0 \mathrm{MGLY}+1.5 \mathrm{HCHO}+1.5 \mathrm{HO}_{2}$ & $1.27 \mathrm{E}-12$ & 0.00 & -360.0 & 3 \\
\hline 103 & $\mathrm{HO}_{2}+\mathrm{MACRO}_{2} \rightarrow \mathrm{MACROOH}$ & $1.82 \mathrm{E}-13$ & 0.00 & -1300.0 & 3 \\
\hline 104 & $\mathrm{MACRO}_{2}+\mathrm{MACRO}_{2} \rightarrow 2 \mathrm{HACET}+2 \mathrm{MGLY}+\mathrm{HCHO}+\mathrm{CO}$ & $1.00 \mathrm{E}-12$ & 0.00 & 0.0 & 3 \\
\hline 105 & $\mathrm{MACRO}_{2}+\mathrm{MACRO}_{2} \rightarrow \mathrm{HO}_{2}+\mathrm{HO}_{2}$ & $1.00 \mathrm{E}-12$ & 0.00 & 0.0 & 3 \\
\hline 106 & $\mathrm{OH}+\mathrm{MPAN} \rightarrow \mathrm{HACET}+\mathrm{NO}_{2}$ & $2.90 \mathrm{E}-11$ & 0.00 & 0.0 & 1 \\
\hline 107 & $\mathrm{OH}+\mathrm{MACROOH} \rightarrow \mathrm{MACRO}_{2}$ & $3.00 \mathrm{E}-11$ & 0.00 & 0.0 & 3 \\
\hline 108 & $\mathrm{OH}+\mathrm{HACET} \rightarrow \mathrm{MGLY}+\mathrm{HO}_{2}$ & $3.00 \mathrm{E}-12$ & 0.00 & 0.0 & 1 \\
\hline 109 & $\mathrm{OH}+\mathrm{MGLY} \rightarrow \mathrm{MeCO}_{3}+\mathrm{CO}$ & $1.50 \mathrm{E}-11$ & 0.00 & 0.0 & 1 \\
\hline 110 & $\mathrm{NO}_{3}+\mathrm{MGLY} \rightarrow \mathrm{MeCO}_{3}+\mathrm{CO}+\mathrm{HONO}_{2}$ & $3.46 \mathrm{E}-12$ & 0.00 & 1860.0 & 2 \\
\hline 111 & $\mathrm{OH}+\mathrm{NALD} \rightarrow \mathrm{HCHO}+\mathrm{CO}+\mathrm{NO}_{2}$ & $4.40 \mathrm{E}-12$ & 0.00 & -365.0 & 1 \\
\hline 112 & $\mathrm{OH}+\mathrm{MeCO}_{3} \mathrm{H} \rightarrow \mathrm{MeCO}_{3}$ & $3.70 \mathrm{E}-12$ & 0.00 & 0.0 & 2 \\
\hline 113 & $\mathrm{OH}+\mathrm{MeCO}_{2} \mathrm{H} \rightarrow \mathrm{MeOO}$ & $4.00 \mathrm{E}-13$ & 0.00 & -200.0 & 3 \\
\hline 114 & $\mathrm{OH}+\mathrm{HCOOH} \rightarrow \mathrm{HO}_{2}$ & $4.50 \mathrm{E}-13$ & 0.00 & 0.0 & 1 \\
\hline
\end{tabular}

Table 10. Additional uni- and termolecular reactions associated with the Mainz Isoprene Mechanism in the UKCA TropIsop chemistry scheme, where MACR = lumped species consisting of methacrolein, methyl vinyl ketone and other $\mathrm{C}_{4}$ carbonyls from isoprene chemistry, and MPAN = peroxy methacrylic nitric anhydride (and other higher PANs from isoprene chemistry). Rate coefficients for termolecular

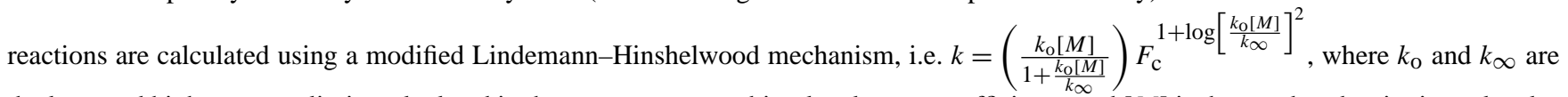
the low- and high-pressure limits calculated in the same manner as bimolecular rate coefficients, and $[M]$ is the number density in molecules $\mathrm{cm}^{-3}$. Reference: (1) Pöschl et al. (2000), and references therein.

\begin{tabular}{llcccccccc}
\hline \multirow{2}{*}{ Label } & \multirow{2}{*}{ Reaction } & \multicolumn{3}{c}{$F_{\mathrm{c}}$} & \multicolumn{3}{c}{ Low-pressure limit, $k_{\mathrm{O}}$} & \multicolumn{3}{c}{ High-pressure limit, $k_{\infty}$} & \multicolumn{2}{c}{ Ref. } \\
& & & $\mathrm{A}$ & $\alpha$ & $\frac{E_{\mathrm{a}}}{R}$ & $\mathrm{~A}$ & $\alpha$ & $\frac{E_{\mathrm{a}}}{R}$ & \\
\hline 14 & $\mathrm{MACRO}_{2}+\mathrm{NO}_{2} \rightarrow \mathrm{MPAN}$ & 0.30 & $2.70 \mathrm{E}-28$ & -7.10 & 0.0 & $1.20 \mathrm{E}-11$ & -0.90 & 0.0 & 1 \\
15 & $\mathrm{MPAN} \rightarrow \mathrm{MACRO}_{2}+\mathrm{NO}_{2}$ & 0.30 & $4.90 \mathrm{E}-03$ & 0.00 & 12100.0 & $5.40 \mathrm{E}+16$ & 0.00 & 13830.0 & 1 \\
\hline
\end{tabular}


Table 11. Summary of the global annual emissions of trace gases used with the two chemistry schemes, StdTrop and TropIsop, in $\mathrm{UKCA}$, where $\mathrm{Me}=\mathrm{CH}_{3}$.

\begin{tabular}{|c|c|c|}
\hline Species & Sector & Emissions \\
\hline \multirow{9}{*}{$\mathrm{C}_{2} \mathrm{H}_{6}$} & Anthropogenic $\left(\mathrm{C}_{2} \mathrm{H}_{6}\right)$ & $3.2 \mathrm{Tg} \mathrm{C}_{2} \mathrm{H}_{6} \mathrm{yr}^{-1}$ \\
\hline & Anthropogenic $\left(\mathrm{C}_{2} \mathrm{H}_{4}\right)$ & $8.0 \mathrm{Tg} \mathrm{C}_{2} \mathrm{H}_{6} \mathrm{yr}^{-1}$ \\
\hline & Anthropogenic $\left(\mathrm{C}_{2} \mathrm{H}_{2}\right)$ & $3.8 \mathrm{Tg} \mathrm{C}_{2} \mathrm{H}_{6} \mathrm{yr}^{-1}$ \\
\hline & Biomass Burning $\left(\mathrm{C}_{2} \mathrm{H}_{6}\right)$ & $3.2 \mathrm{Tg} \mathrm{C}_{2} \mathrm{H}_{6} \mathrm{yr}^{-1}$ \\
\hline & Biomass Burning $\left(\mathrm{C}_{2} \mathrm{H}_{4}\right)$ & $6.8 \mathrm{TgC}_{2} \mathrm{H}_{6} \mathrm{yr}^{-1}$ \\
\hline & Biomass Burning $\left(\mathrm{C}_{2} \mathrm{H}_{2}\right)$ & $1.7 \mathrm{Tg} \mathrm{C}_{2} \mathrm{H}_{6} \mathrm{yr}^{-1}$ \\
\hline & Shipping $\left(\mathrm{C}_{2} \mathrm{H}_{6}\right)$ & $0.2 \mathrm{TgC}_{2} \mathrm{H}_{6} \mathrm{yr}^{-1}$ \\
\hline & Shipping $\left(\mathrm{C}_{2} \mathrm{H}_{4}\right)$ & $0.2 \mathrm{Tg} \mathrm{C}_{2} \mathrm{H}_{6} \mathrm{yr}^{-1}$ \\
\hline & Shipping $\left(\mathrm{C}_{2} \mathrm{H}_{2}\right)$ & $0.0 \mathrm{Tg}_{2} \mathrm{H}_{6} \mathrm{yr}^{-1}$ \\
\hline Total & & $27.1 \mathrm{Tg} \mathrm{C}_{2} \mathrm{H}_{6} \mathrm{yr}^{-1}$ \\
\hline \multirow[t]{6}{*}{$\mathrm{C}_{3} \mathrm{H}_{8}$} & Anthropogenic $\left(\mathrm{C}_{3} \mathrm{H}_{8}\right)$ & $3.4 \mathrm{Tg} \mathrm{C}_{3} \mathrm{H}_{8} \mathrm{yr}^{-1}$ \\
\hline & Anthropogenic $\left(\mathrm{C}_{3} \mathrm{H}_{6}\right)$ & $3.3 \mathrm{Tg} \mathrm{C}_{3} \mathrm{H}_{8} \mathrm{yr}^{-1}$ \\
\hline & Biomass Burning $\left(\mathrm{C}_{3} \mathrm{H}_{8}\right)$ & $2.0 \mathrm{TgC}_{3} \mathrm{H}_{8} \mathrm{yr}^{-1}$ \\
\hline & Biomass Burning $\left(\mathrm{C}_{3} \mathrm{H}_{6}\right)$ & $4.1 \mathrm{Tg} \mathrm{C}_{3} \mathrm{H}_{8} \mathrm{yr}^{-1}$ \\
\hline & Shipping $\left(\mathrm{C}_{3} \mathrm{H}_{8}\right)$ & $0.4 \mathrm{Tg} \mathrm{C}_{3} \mathrm{H}_{8} \mathrm{yr}^{-1}$ \\
\hline & Shipping $\left(\mathrm{C}_{3} \mathrm{H}_{6}\right)$ & $0.3 \mathrm{Tg} \mathrm{C}_{3} \mathrm{H}_{8} \mathrm{yr}^{-1}$ \\
\hline Total & & $13.5 \mathrm{Tg} \mathrm{C}_{3} \mathrm{H}_{8} \mathrm{yr}^{-1}$ \\
\hline \multirow[t]{10}{*}{$\mathrm{CH}_{4}$} & Anthropogenic & $274.1 \mathrm{Tg} \mathrm{CH}_{4} \mathrm{yr}^{-1}$ \\
\hline & Biomass Burning & $25.5 \mathrm{Tg} \mathrm{CH}_{4} \mathrm{yr}^{-1}$ \\
\hline & Shipping & $0.4 \mathrm{TgCH}_{4} \mathrm{yr}^{-1}$ \\
\hline & Termites & $20.0 \mathrm{Tg} \mathrm{CH}_{4} \mathrm{yr}^{-1}$ \\
\hline & Oceans & $15.0 \mathrm{Tg} \mathrm{CH}_{4} \mathrm{yr}^{-1}$ \\
\hline & Hydrates & $10.0 \mathrm{TgCH}_{4} \mathrm{yr}^{-1}$ \\
\hline & Wetlands & $108.7 \mathrm{Tg} \mathrm{CH}_{4} \mathrm{yr}^{-1}$ \\
\hline & Bogs & $30.0 \mathrm{Tg} \mathrm{CH}_{4} \mathrm{yr}^{-1}$ \\
\hline & Swamps & $39.1 \mathrm{Tg} \mathrm{CH}_{4} \mathrm{yr}^{-1}$ \\
\hline & Tundra & $3.2 \mathrm{Tg} \mathrm{CH}_{4} \mathrm{yr}^{-1}$ \\
\hline Total & & $526.0 \mathrm{Tg} \mathrm{CH}_{4} \mathrm{yr}^{-1}$ \\
\hline \multirow[t]{5}{*}{$\mathrm{CO}$} & Anthropogenic & $607.5 \mathrm{Tg} \mathrm{CO} \mathrm{yr}^{-1}$ \\
\hline & Biomass Burning & $459.1 \mathrm{Tg} \mathrm{CO} \mathrm{yr}^{-1}$ \\
\hline & Shipping & $1.2 \mathrm{Tg} \mathrm{CO} \mathrm{yr}^{-1}$ \\
\hline & Oceans & $45.0 \mathrm{Tg} \mathrm{CO} \mathrm{yr}^{-1}$ \\
\hline & * Vegetation & $354.0 \mathrm{Tg} \mathrm{CO} \mathrm{yr}^{-1}$ \\
\hline Total & & $1466.8 \mathrm{Tg} \mathrm{CO} \mathrm{yr}^{-1}$ \\
\hline \multirow[t]{2}{*}{$\mathrm{HCHO}$} & Anthropogenic & $3.2 \mathrm{Tg} \mathrm{HCHO} \mathrm{yr}^{-1}$ \\
\hline & Biomass Burning & $5.8 \mathrm{Tg} \mathrm{HCHO} \mathrm{yr}^{-1}$ \\
\hline Total & & 9.0 Tg HCHO yr ${ }^{-1}$ \\
\hline \multirow[t]{6}{*}{$\mathrm{NO}_{\mathrm{x}}$} & Anthropogenic & $26.5 \mathrm{Tg} \mathrm{N} \mathrm{yr}^{-1}$ \\
\hline & Biomass Burning & $4.5 \mathrm{Tg} \mathrm{N} \mathrm{yr}^{-1}$ \\
\hline & Shipping & 5.4 $\mathrm{Tg} \mathrm{N} \mathrm{yr}^{-1}$ \\
\hline & Soil & 5.6 $\mathrm{Tg} \mathrm{N} \mathrm{yr}^{-1}$ \\
\hline & Aircraft & $0.8 \mathrm{Tg} \mathrm{N} \mathrm{yr}^{-1}$ \\
\hline & Lightning & $<2.0 \mathrm{Tg} \mathrm{Nyr}^{-1}$ \\
\hline Total & & $<44.8 \mathrm{Tg} \mathrm{Nyr}^{-1}$ \\
\hline $\mathrm{MeCHO}$ & Biomass Burning & 8.7 ${\mathrm{Tg} \mathrm{MeCHO} \mathrm{yr}^{-1}}^{-1}$ \\
\hline Total & & $8.7 \mathrm{Tg} \mathrm{MeCHO} \mathrm{yr}^{-1}$ \\
\hline \multirow[t]{3}{*}{$\mathrm{Me}_{2} \mathrm{CO}$} & Anthropogenic & $0.7 \mathrm{Tg} \mathrm{Me}_{2} \mathrm{CO} \mathrm{yr}^{-1}$ \\
\hline & Biomass Burning & 5.0 $\mathrm{Tg} \mathrm{Me}_{2} \mathrm{CO} \mathrm{yr}{ }^{-1}$ \\
\hline & Vegetation & $40.0 \mathrm{Tg} \mathrm{Me}_{2} \mathrm{CO} \mathrm{yr}^{-1}$ \\
\hline Total & & $45.7 \mathrm{Tg} \mathrm{Me}_{2} \mathrm{CO}_{\mathrm{yr}^{-1}}$ \\
\hline${ }^{*} \mathrm{C}_{5} \mathrm{H}_{8}$ & Vegetation & $503.0 \mathrm{Tg} \mathrm{Cyr}^{-1}$ \\
\hline Total & & $503.0 \mathrm{Tg} \mathrm{C} \mathrm{yr}^{-1}$ \\
\hline
\end{tabular}

* In the case of the StdTrop chemistry scheme, an additional $354 \mathrm{Tg} \mathrm{CO} \mathrm{yr}^{-1}$ are added as a surrogate for $\mathrm{C}_{5} \mathrm{H}_{8}$ emissions. When running with the TropIsop chemistry scheme, these additional emissions are neglected, but $\mathrm{C}_{5} \mathrm{H}_{8}$ emissions with a global annual emission total of $503 \mathrm{Tg} \mathrm{Cyr}^{-1}$ are added instead.
The standard tropospheric chemistry scheme, StdTrop, takes account of surface emissions of $\mathrm{C}_{2} \mathrm{H}_{6}, \mathrm{C}_{3} \mathrm{H}_{8}, \mathrm{CH}_{4}$, $\mathrm{CO}, \mathrm{HCHO}, \mathrm{NO}_{\mathrm{x}}, \mathrm{CH}_{3} \mathrm{CHO}$, and $\mathrm{CH}_{3} \mathrm{COCH}_{3}$. A summary is provided in Table 11. For $\mathrm{C}_{2} \mathrm{H}_{6}$ and $\mathrm{C}_{3} \mathrm{H}_{8}$, it was decided to lump all $\mathrm{C} 2\left(\mathrm{C}_{2} \mathrm{H}_{6}\right.$, ethene $\left(\mathrm{C}_{2} \mathrm{H}_{4}\right)$, and ethyne $\left.\left(\mathrm{C}_{2} \mathrm{H}_{2}\right)\right)$ and $\mathrm{C} 3\left(\mathrm{C}_{3} \mathrm{H}_{8}\right.$ and propene $\left.\left(\mathrm{C}_{3} \mathrm{H}_{6}\right)\right)$ species together and treat them as emissions of $\mathrm{C}_{2} \mathrm{H}_{6}$ and $\mathrm{C}_{3} \mathrm{H}_{8}$, respectively. As well as emissions from land-based anthropogenic sources, biomass burning and shipping (Lamarque et al., 2010), natural emissions of $\mathrm{CH}_{4}$ were also included. Those from termites, wetlands, swamps, bogs, and tundra are from Fung et al. (1991), while oceanic and hydrate emissions are distributed temporally and spatially using distributions from Global Emissions Inventory Activity (GEIA; http://www.geiacenter.org/inventories/present.html).

For CO, emissions from Lamarque et al. (2010) are combined with oceanic emissions of $\mathrm{CO}$, whose spatial and temporal distribution were provided by the Global Emissions Inventory Activity (GEIA; http://www.geiacenter.org/ inventories/present.html) and are based on distributions of oceanic VOC emissions from Guenther et al. (1995). In the absence of a $\mathrm{C}_{5} \mathrm{H}_{8}$ degradation scheme, an additional $354 \mathrm{Tg} \mathrm{CO} \mathrm{yr}^{-1}$ was added, based on a global mean $\mathrm{CO}$ yield of $30 \%$ from $\mathrm{C}_{5} \mathrm{H}_{8}$ from a study by Pfister et al. (2008) and a global $\mathrm{C}_{5} \mathrm{H}_{8}$ emission source of $503 \mathrm{Tg} \mathrm{C} \mathrm{yr}^{-1}$ (Guenther et al., 1995). It is distributed spatially and temporally based on $\mathrm{C}_{5} \mathrm{H}_{8}$ emissions from Guenther et al. (1995). For HCHO emissions, land-based anthropogenic sources and biomass burning contribute to the global annual total emissions of $9.0 \mathrm{Tg} \mathrm{HCHO} \mathrm{yr}^{-1}$.

For $\mathrm{NO}_{\mathrm{x}}$ surface emissions, contributions from landbased anthropogenic sources, biomass burning, and shipping from Lamarque et al. (2010) are used. Added to these are a contribution from natural soil emissions, based on a global and monthly distribution provided by GEIA (http:// www.geiacenter.org/inventories/present.html), and based on the global empirical model of soil-biogenic $\mathrm{NO}_{\mathrm{x}}$ emissions from Yienger and Levy (1995). For $\mathrm{CH}_{3} \mathrm{CHO}$, the NMVOC biomass burning emissions for the period 19972006 from Lamarque et al. (2010) are used. Using emission factors from Andreae and Merlet (2001) for grass fires, tropical forest fires, and extra-tropical forest fires, emissions of NMVOCs are converted into emissions of $\mathrm{CH}_{3} \mathrm{CHO}$, giving a global annual total of $8.7 \mathrm{TgCH}_{3} \mathrm{CHO} \mathrm{yr}^{-1}$. Finally, emissions of $\mathrm{CH}_{3} \mathrm{COCH}_{3}$ are added from land-based anthropogenic sources and biomass burning from Lamarque et al. (2010), giving a global annual emission total of $5.7 \mathrm{Tg} \mathrm{CH}_{3} \mathrm{COCH}_{3} \mathrm{yr}^{-1}$. The dominant source of $\mathrm{CH}_{3} \mathrm{COCH}_{3}$, however, is from vegetation through direct emissions, production from decomposing plant matter as well as production from terpene oxidation. A global emission strength of $40.0 \mathrm{Tg} \mathrm{CH}_{3} \mathrm{COCH}_{3} \mathrm{yr}^{-1}$ is used here following von Kuhlmann et al. (2003), which are distributed spatially and temporally based on distributions from Guenther et al. (1995). For the TropIsop chemistry scheme, the 
emissions are identical to those used by StdTrop except that the additional $\mathrm{CO}$ emissions from $\mathrm{C}_{5} \mathrm{H}_{8}$ oxidation are omitted; instead, a global annual $\mathrm{C}_{5} \mathrm{H}_{8}$ emission source of $503 \mathrm{Tg} \mathrm{C} \mathrm{yr}^{-1}$ (Guenther et al., 1995) is prescribed, onto which a diurnal cycle is imposed based on local solar zenith angle. The use of interactive biogenic emissions in UKCA will be discussed by Folberth et al. (2014) in Part 4 of the UKCA paper series.

In addition to surface emissions, three-dimensional emissions of $\mathrm{NO}_{\mathrm{x}}$ from aircraft and lightning are also taken into account. Aircraft emissions are taken from the historical data set compiled by Lamarque et al. (2010), are concentrated in the Northern Hemisphere, and are strongest in the upper troposphere/lower stratosphere (UTLS) region. Lightning emissions of $\mathrm{NO}_{\mathrm{x}}$ are also added to UKCA, based on the distribution and frequency of lightning flashes following Price and Rind (1992, 1993, 1994), where the flash frequency is related to the height of the climate model's convective cloud top. Emissions are added from the surface up to $500 \mathrm{hPa}$ for cloud-to-ground (CG) flashes and from $500 \mathrm{hPa}$ up to the convective cloud top for intracloud (IC) flashes and are related to the discharge energy, with the energy from a CG flash being 10 times higher than that from an IC flash (Price et al., 1997). A scaling factor was also applied to give a global annual emission total of $5 \mathrm{Tg} \mathrm{N} \mathrm{yr}^{-1}$. However, this scaling had been evaluated at an early stage of the model development, leading to emissions that were inadvertently too low in this model configuration, i.e. $<2 \mathrm{TgN} \mathrm{yr}^{-1}$ (Young et al., 2013).

\subsection{Boundary conditions}

In the tropospheric configuration of UKCA using the L38 climate model, the chemistry is calculated interactively up to the model lid at $39 \mathrm{~km}$. Nevertheless, boundary conditions are applied at the top of the model's domain to take account of missing stratospheric processes, such as chlorine and bromine chemistry. The location of a combined isentropic-dynamical tropopause (Hoerling et al., 1993) is diagnosed on a timestep-by-timestep basis. Then, modelled $\mathrm{O}_{3}$ from UKCA is overwritten in all model levels which are $3-4 \mathrm{~km}$ above the tropopause using the time-varying $\mathrm{O}_{3}$ field that is normally used to drive the climate model's radiation scheme in the absence of chemistry. In the case of the chemistry-climate simulations here, stratospheric $\mathrm{O}_{3}$ was that recommended for CMIP5 (Cionni et al., 2011). For $\mathrm{NO}_{\mathrm{y}}$, a fixed $\mathrm{O}_{3}$ to $\mathrm{HNO}_{3}$ ratio of $1.0 / 1000.0 \mathrm{~kg} \mathrm{~N} / \mathrm{kg} \mathrm{O}_{3}$ from Murphy and Fahey (1994) is applied to $\mathrm{HNO}_{3}$ on the same model levels where $\mathrm{O}_{3}$ is being overwritten. For $\mathrm{CH}_{4}$, an explicit loss term, scaled to give a global annual loss of approximately $40 \mathrm{Tg} \mathrm{CH}_{4} \mathrm{yr}^{-1}$, is applied in the 3 uppermost levels to account for stratospheric oxidation.

In addition to running with $\mathrm{CH}_{4}$ fully interactive, UKCA also has the option of running with a prescribed global surface $\mathrm{CH}_{4}$ concentration; this can be a time-invariant or time-varying concentration, depending on the type of model integration being carried out. In the HadGEM2-UKCA coupled model simulations being evaluated here, $\mathrm{CH}_{4}$ was fully interactive.

\section{Model evaluation}

The UKCA configuration described here supports two chemistry schemes, StdTrop and TropIsop, and differences between the performance of the two chemistry schemes will be discussed further in Sect. 6. The model evaluation here, however, will focus mainly on StdTrop. This was the chemistry scheme that formed part of the Met Office Hadley Centre's ESM HadGEM2-ES (Collins et al., 2011) and was used in all the HadGEM2-ES centennial simulations submitted to CMIP5 (Jones et al., 2011). A total of $10 \mathrm{yr}$ has been simulated using the StdTrop chemistry scheme, with initial concentrations taken from a previous integration of UKCA in which $\mathrm{CH}_{4}$ was spun up to a steady state. Surface and aircraft emissions for the year 2000 were used repeatedly and all $10 \mathrm{yr}$ are used here in the model evaluation. Although the UKCA model simulates over 40 species, the discussion here focuses on key species such as $\mathrm{CH}_{4}, \mathrm{CO}, \mathrm{NO}_{\mathrm{x}}, \mathrm{OH}$, and $\mathrm{O}_{3}$, which directly govern the oxidising capacity of the troposphere.

\subsection{Methane $\left(\mathrm{CH}_{4}\right)$}

Multi-annual surface and zonal mean plots of $\mathrm{CH}_{4}$ from the StdTrop chemistry scheme can be seen in Fig. 12a and b. They indicate that surface concentrations of $\mathrm{CH}_{4}$ are very homogeneous in the Southern Hemisphere, with concentrations typically in the 1700-1750 ppbv range, except for higher concentrations in Africa and South America associated with anthropogenic sources, mainly from the agricultural sector, and natural emissions. In the Northern Hemisphere, variability is high over the continental regions. Over Canada and Alaska, for example, enhanced concentrations can be attributed principally to wetland emissions. Over North America, on the other hand, the dominant source of $\mathrm{CH}_{4}$ emissions are of anthropogenic origin (agriculture, energy production, and waste treatment) and to a lesser extent, natural emissions from swamps, bogs, and tundra. The largest enhancements in surface concentration above the global mean are over India and Southeast Asia; emissions from these regions are of anthropogenic origin (agriculture, energy, waste treatment, and domestic sectors). Less variability is evident over the Northern Hemisphere oceans, away from continental emissions sources and where $\mathrm{CH}_{4}$ can be well mixed. The global annual mean surface concentration from the StdTrop UKCA simulation coupled to HadGEM2 is $1797 \mathrm{ppbv}$, within $3 \%$ of the globally and annually averaged observed concentration for the year 2000 of 1751 ppbv (Dlugokencky et al., 2003). 

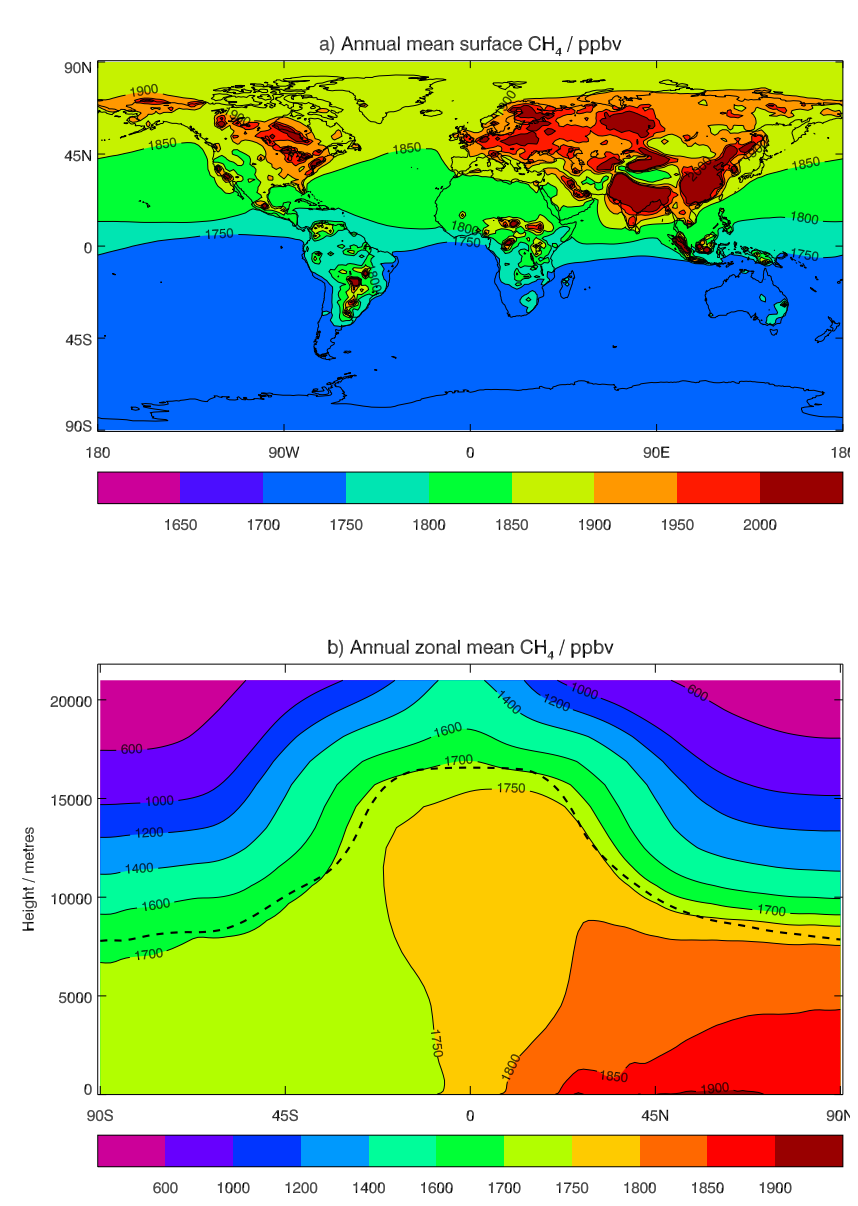

Fig. 12. Multi-annual (a) surface and (b) zonal mean $\mathrm{CH}_{4}$ distributions from the StdTrop chemistry scheme in the tropospheric configuration of the UKCA model. The thick dashed line in (b) marks the position of the modelled tropopause (Hoerling et al., 1993).

The multi-annual zonal mean distribution, shown in Fig. $12 \mathrm{~b}$, is characterized by high $\mathrm{CH}_{4}$ concentrations near the surface in the Northern Hemisphere mid and high latitudes, with decreasing concentrations aloft. In the Southern Hemisphere, the entire troposphere is very well mixed with concentrations similar to surface concentrations (i.e. in the $1700-1750 \mathrm{ppbv}$ range). In the tropics, there is evidence of efficient upward transport from the surface to the upper troposphere due to convection as well as some indication of inter-hemispheric transport. There is a strong vertical gradient above the tropopause, although it appears to be too strong in comparison with the zonal mean distribution from the MOZART model (Hauglustaine et al., 1998; Park et al., 2004) and from concentrations derived from Halogen Occultation Experiment (HALOE) satellite observations (Park et al., 2004). Results from a comparison between UKCA and SCIAMACHY also support this (Hayman et al., 2014). This steep decline in $\mathrm{CH}_{4}$ was also evident in the stratospheric configuration of UKCA (Morgenstern et al., 2009), particularly in the tropics, and was attributed to slow ascent in the tropical pipe. In the HadGEM2-UKCA coupled configuration, it could also be partly due to the entire stratospheric removal of $\mathrm{CH}_{4}$ occurring at too low an altitude in comparison with interactive removal in a model with an extended top.

The total $\mathrm{CH}_{4}$ burden, calculated as a multi-annual mean from the $10 \mathrm{yr}$ of model integration, is found to be $4722 \mathrm{Tg} \mathrm{CH}_{4}$, reasonably comparable to the total burden of $4850 \mathrm{Tg} \mathrm{CH}_{4}$ for the year 1998 calculated by Prather et al. (2001). Of the total model burden of $4722 \mathrm{TgCH}_{4}$, $3984 \mathrm{Tg} \mathrm{CH}_{4}$ of it resides below the diagnosed model tropopause with the remaining $738 \mathrm{Tg} \mathrm{CH}_{4}$ aloft. The only source of atmospheric $\mathrm{CH}_{4}$ in the model is from surface emissions (Table 11), totalling $526 \mathrm{Tg} \mathrm{CH}_{4} \mathrm{yr}^{-1}$ for the year 2000. It is removed from the atmosphere by tropospheric oxidation by $\mathrm{OH}$, which in the model accounts for $420 \pm 3.4 \mathrm{Tg} \mathrm{CH}_{4} \mathrm{yr}^{-1}$. The atmospheric lifetime associated with the tropospheric $\mathrm{CH}_{4}$ burden and its loss by $\mathrm{OH}$ is $9.96 \mathrm{yr}$, which is in excellent agreement with the estimate of $9.6 \mathrm{yr}$ from the Intergovernmental Panel on Climate Change (IPCC) Third Assessment Report (TAR) by Prather et al. (2001). It also agrees with a more recent intercomparison of chemistry models by Stevenson et al. (2006), in which the lifetime against $\mathrm{OH}$ loss was calculated to be $9.6 \pm 0.4 \mathrm{yr}$. In HadGEM2-UKCA, the stratospheric removal and soil sink account for approximately 68 and $50 \mathrm{TgCH}_{4} \mathrm{yr}^{-1}$. These are larger than estimated by Prather et al. (2001), although there is significant interannual variability, particularly in the strength of the stratospheric loss (i.e. $\pm 10 \mathrm{Tg} \mathrm{CH}_{4} \mathrm{yr}^{-1}$ ). This budget was calculated using all the years from the 10-yr simulation. However, there was little sensitivity to the choice of years, confirming that $\mathrm{CH}_{4}$ was indeed in steady state.

A comparison between the modelled surface $\mathrm{CH}_{4}$ concentrations from the StdTrop chemistry integration and observations from the Earth Systems Research Laboratory's Global Monitoring Division of the National Oceanic and Atmospheric Administration (http://www.esrl.noaa.gov/gmd/) can be seen in Fig. 13. The particular stations used were chosen on the basis of their long measurement records (for $\mathrm{CH}_{4}$ in addition to $\mathrm{O}_{3}$ and $\mathrm{CO}$ ) although only observations from the 1999-2006 time period are used here. This is because $\mathrm{CH}_{4}$ observations suggest that atmospheric levels were fairly constant during this period apart from a small increase in 2002 to 2003 (Dlugokencky et al., 2009). Statistical measures of skill at each location, such as relative annual mean bias, root mean square error, correlation coefficient, and model score, are also included in the figure. The comparison indicates that the StdTrop chemistry scheme in UKCA captures the seasonal cycle and absolute concentrations of surface $\mathrm{CH}_{4}$ at most stations very well (e.g. Niwot Ridge has a root mean square error and a correlation coefficient of $9.5 \mathrm{ppbv}$ and 0.94 , respectively, while Mauna Loa has values of $21.1 \mathrm{ppbv}$ and 0.85). The most notable exception to this is at Barrow $\left(71^{\circ} \mathrm{N}\right.$, $157^{\circ} \mathrm{W}$ ), where the relative annual mean bias is low at $2.3 \%$ but the correlation coefficient is -0.63 and the root mean square error is $75.8 \mathrm{ppbv}$. Modelled surface concentrations 

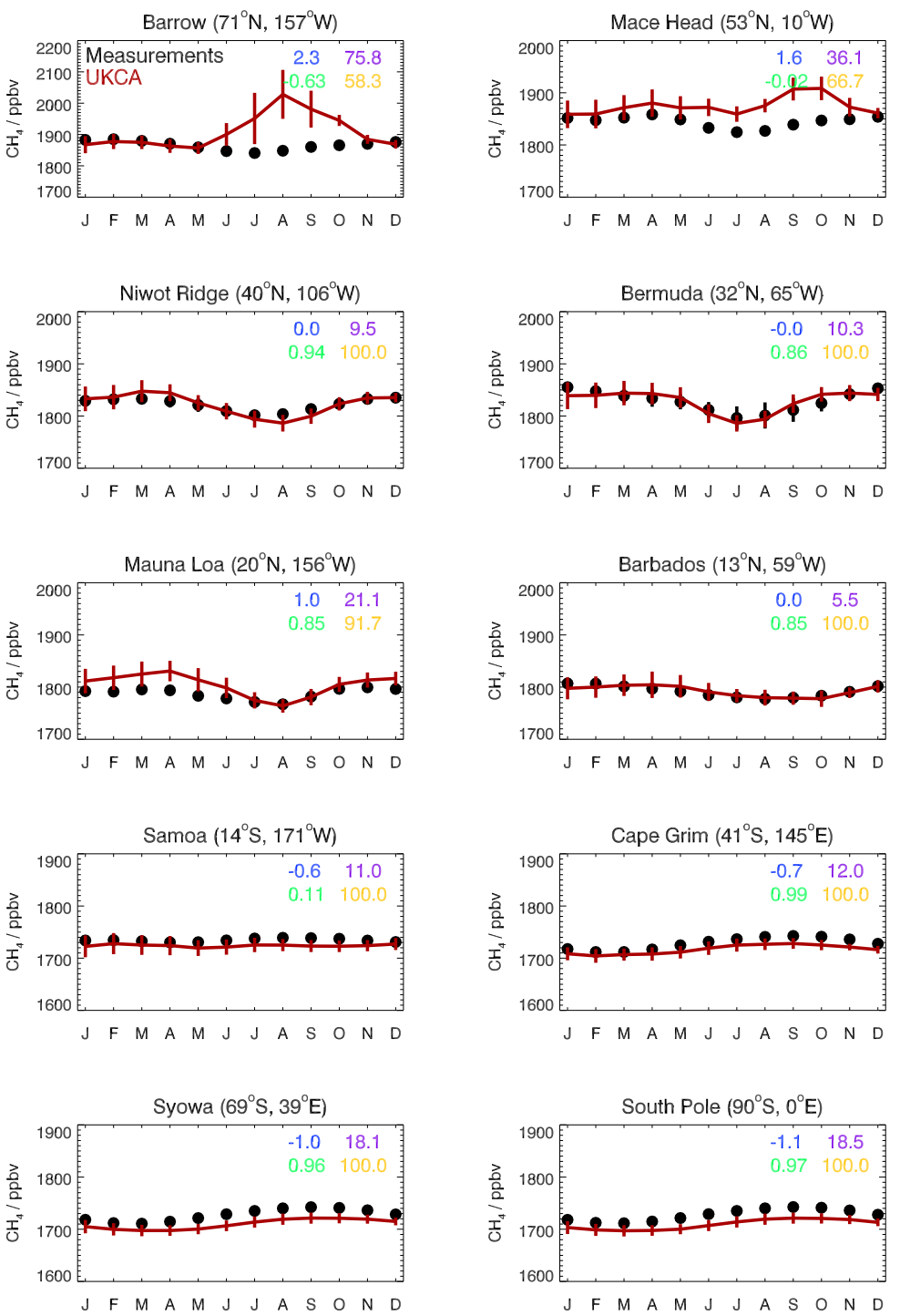

Fig. 13. Comparison between multi-annual monthly mean modelled surface $\mathrm{CH}_{4}$ concentrations with surface observations from the 1999 2006 time period. The surface sites include Barrow $\left(71^{\circ} \mathrm{N}, 157^{\circ} \mathrm{W}\right)$, Mace Head $\left(53^{\circ} \mathrm{N}, 10^{\circ} \mathrm{W}\right)$, Niwot Ridge $\left(40^{\circ} \mathrm{N}, 106^{\circ} \mathrm{W}\right)$, Bermuda $\left(32^{\circ} \mathrm{N}, 65^{\circ} \mathrm{W}\right)$, Mauna Loa $\left(20^{\circ} \mathrm{N}, 156^{\circ} \mathrm{W}\right)$, Barbados ( $\left.13^{\circ} \mathrm{N}, 59^{\circ} \mathrm{W}\right)$, Samoa $\left(14^{\circ} \mathrm{S}, 171^{\circ} \mathrm{W}\right)$, Cape Grim $\left(41^{\circ} \mathrm{S}, 145^{\circ} \mathrm{E}\right), \mathrm{Syowa}^{\circ}\left(69^{\circ} \mathrm{S}\right.$, $39^{\circ} \mathrm{E}$ ), and the South Pole $\left(90^{\circ} \mathrm{S}, 0^{\circ} \mathrm{E}\right)$. The relative annual mean bias (in \%), the root mean square error (in pbbv), the correlation coefficient, and the model score (the percentage of months where the modelled and observed monthly mean concentrations are within $2 \%$ of each other; in \%) are shown for each location in blue, purple, green, and orange, respectively.

are overestimated by approximately $10 \%$ during the Northern Hemisphere summer and autumn. A similar positive bias, albeit reduced, is also evident in the comparison at Mace Head. A study by Wang et al. (2004) found that the significant summer/autumn positive bias in a priori modelled surface concentrations from the GEOS-CHEM model simulation at Barrow was greatly reduced by their inversion and was specifically due to decreases in the animal, fossil fuel, waste, bogs, and tundra emission sources located in the Northern Hemisphere middle and high latitudes. More recent work by Hayman et al. (2014) also supports the hypothesis that the strength of the Fung et al. (1991) wetland emissions in the Northern Hemisphere (as used in this study) is overstated.

Latitudinal profiles of surface concentrations of $\mathrm{CH}_{4}$ from GLOBALVIEW-CH $_{4}$ (2009) and the HadGEM2-UKCA simulation can be found in Fig. 14. It indicates a clear inter-hemispheric difference with maximum concentrations in the northern mid-latitudes and minimum concentrations in the Southern Hemisphere. There is also some variability in the modelled concentrations in the Northern Hemisphere which is not evident in the observations. In contrast, HadGEM2-UKCA and the observations are consistent 


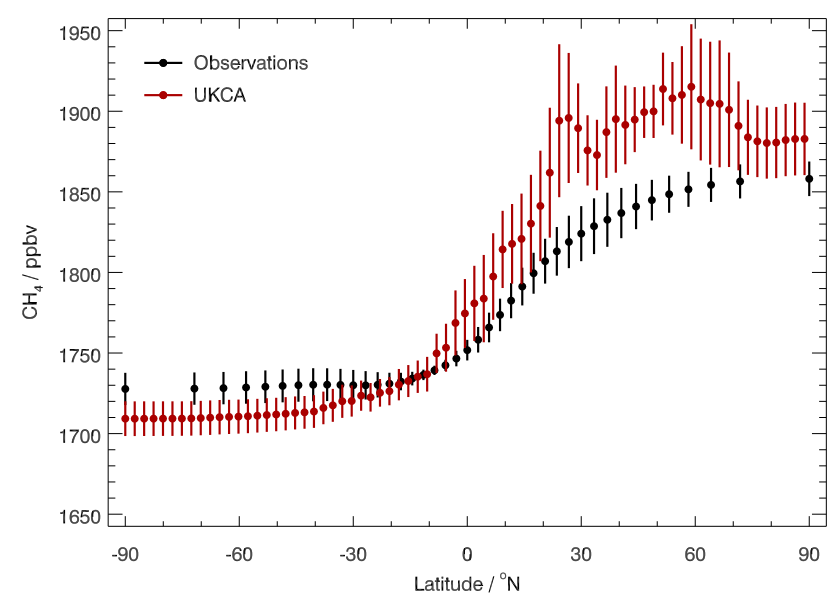

Fig. 14. Comparison between multi-annual latitudinal profiles of observed and modelled surface $\mathrm{CH}_{4}$ concentrations with surface observations from GLOBALVIEW- $\mathrm{CH}_{4}$ (2009). Every second model latitude is omitted from the plot for the purpose of clarity.

in terms of variability in the Southern Hemisphere. Modelled concentrations of surface $\mathrm{CH}_{4}$ are overestimated in the Northern Hemisphere by about $3 \%$ and underestimated in the Southern Hemisphere by approximately $1 \%$, resulting in a modelled inter-hemispheric difference that is greater than that observed. These overestimates and underestimates can have implications for the accuracy of source estimates by inverse modelling (e.g. Houweling et al., 1999; Chen and Prinn, 2006) and may reflect the long inter-hemispheric transport timescale in HadGEM2-UKCA relative to other models, as indicated in Sect. 3.6. However, the absolute concentrations of the model are reasonably comparable with the observations, given that the uncertainty in the strength of the global $\mathrm{CH}_{4}$ source and sink is of the order of $\pm 15 \%$ (Prather et al., 2001; Denman et al., 2007). These results clearly demonstrate the ability of the HadGEM2-UKCA coupled model to simulate $\mathrm{CH}_{4}$ interactively and therefore, has the potential to become a very useful tool in the investigation of feedbacks in the ES involving $\mathrm{CH}_{4}$ (e.g. O'Connor et al., 2010).

\subsection{Carbon monoxide (CO)}

Carbon monoxide (CO), together with $\mathrm{CH}_{4}$, is a primary sink for $\mathrm{OH}$ and $\mathrm{CO}$ is an important tropospheric $\mathrm{O}_{3}$ precursor, thereby playing a significant role in tropospheric chemistry. The dominant source of $\mathrm{CO}$ to the atmosphere is at the surface from incomplete combustion associated with both fossil fuel and biomass burning. However, $\mathrm{CO}$ is also produced by oxidation of $\mathrm{CH}_{4}$ and NMVOCs. Multi-annual surface and zonal mean plots of CO from the StdTrop chemistry scheme can be seen in Fig. 15a and b. The surface plot shows a very pronounced hemispheric asymmetry, with maximum concentrations over the continental regions of the Northern Hemisphere and minimum concentrations over
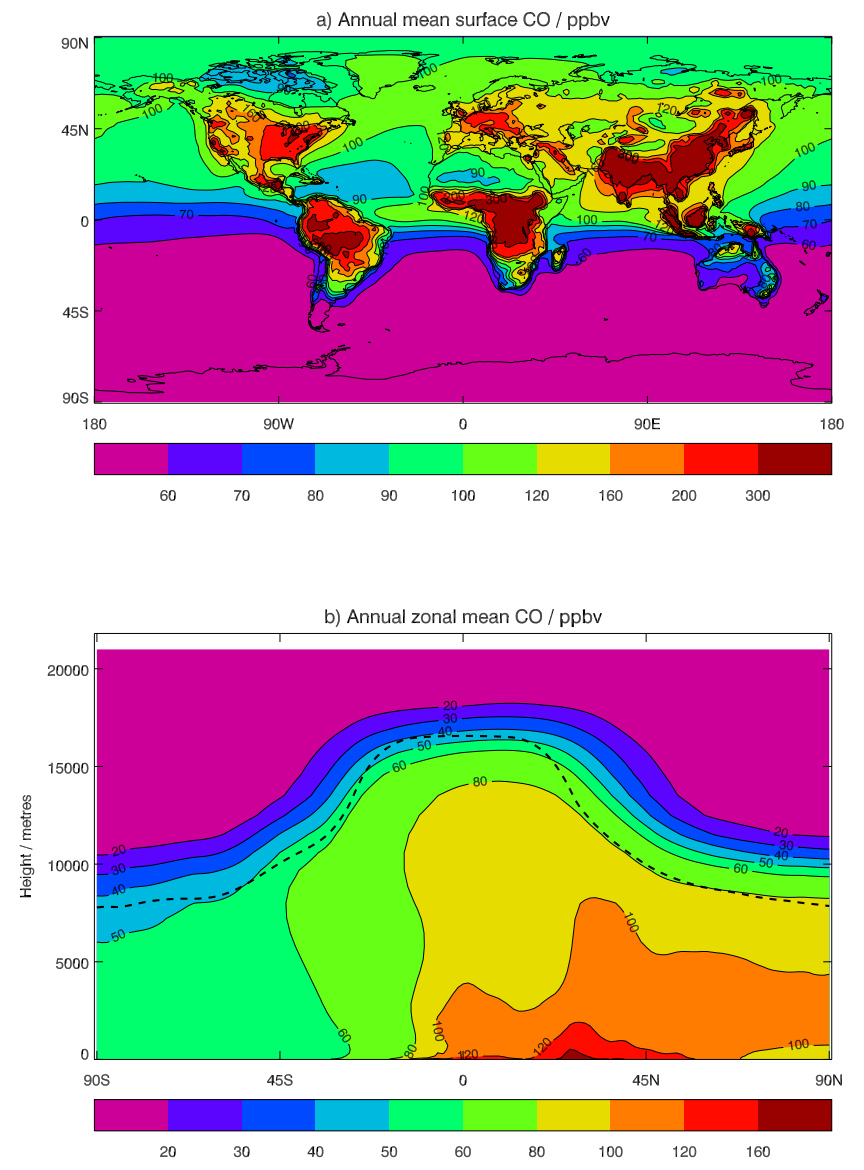

Fig. 15. Multi-annual (a) surface and (b) zonal mean CO distributions from the StdTrop chemistry scheme in the tropospheric configuration of the UKCA model. The thick dashed line in (b) marks the position of the modelled tropopause (Hoerling et al., 1993).

the Southern Hemisphere, particularly over the oceans and Antarctica. The highest concentrations are seen over south and east Asia (over $300 \mathrm{ppbv}$ ), Africa (over $300 \mathrm{ppbv}$ ), South America (over $300 \mathrm{ppbv}$ ), North America (over $200 \mathrm{ppbv}$ ), and Europe (over 200 ppbv). In South America and Africa, the dominant sources of $\mathrm{CO}$ are due to biomass burning and the BVOC surrogate emissions, with a smaller contribution from anthropogenic sources. Over North America, Europe, and south and east Asia, the high modelled surface concentrations are largely attributable to anthropogenic emissions, although there is also a contribution from the surrogate BVOC emissions over the southeast United States and southeast Asia. The spatial heterogeneity, even within the Northern Hemisphere, evident from the HadGEM2-UKCA StdTrop surface distribution is also supported by other model studies (e.g. Hauglustaine et al., 2004). Minimum concentrations of less than $60 \mathrm{ppbv}$ are evident over the oceanic regions of the Southern Hemisphere and are consistent with other models (e.g. Lawrence et al., 1999; Sudo et al., 2002). 

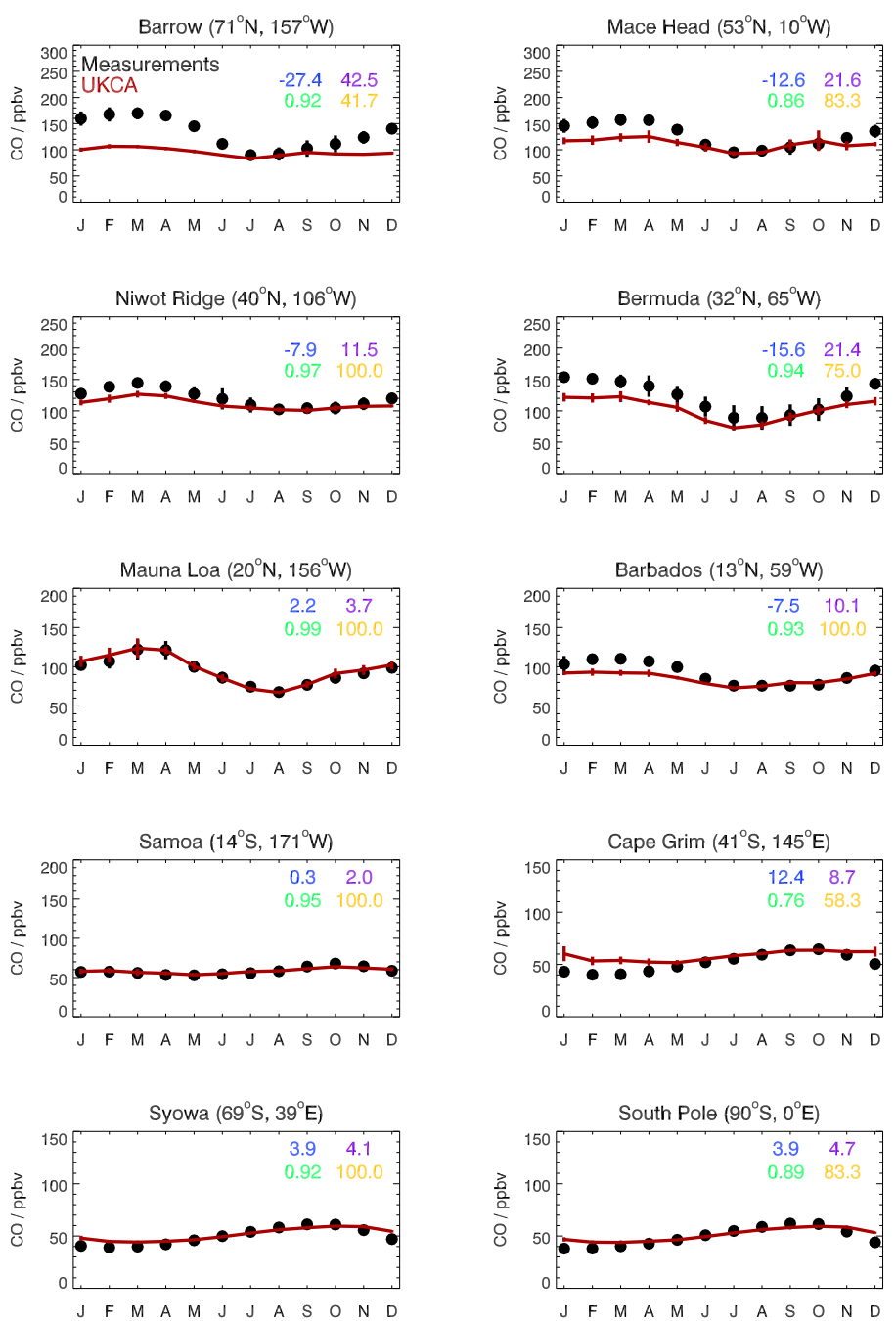

Fig. 16. Comparison between multi-annual monthly mean modelled surface CO concentrations with surface observations from the $1999-2006$ time period. The surface sites include Barrow $\left(71^{\circ} \mathrm{N}, 157^{\circ} \mathrm{W}\right)$, Mace Head $\left(53^{\circ} \mathrm{N}, 10^{\circ} \mathrm{W}\right)$, Niwot Ridge $\left(40^{\circ} \mathrm{N}, 106^{\circ} \mathrm{W}\right)$, Bermuda $\left(32^{\circ} \mathrm{N}\right.$, $\left.65^{\circ} \mathrm{W}\right)$, Mauna Loa $\left(20^{\circ} \mathrm{N}, 156^{\circ} \mathrm{W}\right)$, Barbados ( $\left.13^{\circ} \mathrm{N}, 59^{\circ} \mathrm{W}\right)$, Samoa $\left(14^{\circ} \mathrm{S}, 171^{\circ} \mathrm{W}\right)$, Cape Grim $\left(41^{\circ} \mathrm{S}, 145^{\circ} \mathrm{E}\right), \mathrm{Syowa}\left(69^{\circ} \mathrm{S}, 39^{\circ} \mathrm{E}\right)$, and the South Pole $\left(90^{\circ} \mathrm{S}, 0^{\circ} \mathrm{E}\right)$. The relative annual mean bias (in \%), the root mean square error (in ppbv), the correlation coefficient, and the model score (the percentage of months where the modelled and observed monthly mean concentrations are within $20 \%$ of each other; in $\%)$ are shown for each location in blue, purple, green, and orange, respectively.

The multi-annual zonal mean distribution in Fig. 15b indicates that maximum concentrations occur at the surface in the tropics and mid-latitudes of the Northern Hemisphere, with the maximum being more pronounced in the Northern Hemisphere winter than in the Northern Hemisphere summer. This seasonality in the maximum concentrations was also evident in other models (e.g. Müller and Brasseur, 1995; Hauglustaine et al., 1998) and is attributed to the seasonality of the efficiency of the $\mathrm{CO}+\mathrm{OH}$ reaction. $\mathrm{CO}$ concentrations decrease above the surface in the Northern Hemisphere and the vertical gradient is stronger during the Northern Hemisphere winter than in summer. By contrast, the troposphere in the mid and high latitudes of the Southern Hemisphere is well mixed with typical concentrations of $50-60 \mathrm{ppbv}$ in the annual mean. At the tropopause, there is a strong vertical gradient, particularly in the Northern Hemisphere, with minimum concentrations (below $20 \mathrm{ppbv}$ ) above 18 and $10 \mathrm{~km}$ in the tropics and extra-tropics, respectively. This can be attributed to the relatively higher vertical resolution in HadGEM2 in comparison with other models (e.g. Lawrence et al., 1999). As was the case for $\mathrm{CH}_{4}$, there is evidence of convective uplift, with a secondary maximum in the tropical upper troposphere. There is also a relative minimum in the $\mathrm{CO}$ concentration in the lower troposphere at $20^{\circ} \mathrm{N}$. Both of these features are evident in other model simulations (e.g. Müller and Brasseur, 1995; Hauglustaine et al., 1998) and their existence 
is supported by aircraft observations taken during vertical profiles along meridional cross sections from $70^{\circ} \mathrm{N}-60^{\circ} \mathrm{S}$ (Marenco et al., 1995).

Figure 16 shows a comparison between modelled surface CO concentrations from the StdTrop UKCA chemistry scheme coupled to HadGEM2 with climatological observations from selected sites from NOAA's Earth Systems Research Laboratory's Global Monitoring Division (http:// www.esrl.noaa.gov/gmd/). Statistical measures of skill (relative annual mean bias, root mean square error, correlation coefficient, and model score) are also included in the figure. On the whole, the model captures both the seasonal cycle and the absolute surface concentrations reasonably well - most locations show relative annual mean biases of $15 \%$ or less and correlation coefficients of greater than 0.8. At Barrow, however, the model underestimates the enhanced surface concentrations observed during the Northern Hemisphere winter and spring by $50 \mathrm{ppbv}$ or more. There is also some evidence of a similar winter-spring underestimate at other Northern Hemisphere surface sites (e.g. Mace Head, Bermuda). Using identical anthropogenic emissions, both the G-PUCCINI (Shindell et al., 2006) and the CAM-Chem (Lamarque et al., 2008; Gent et al., 2010) models in the recent Lamarque et al. (2010) study indicate a negative bias and a reduced seasonal cycle in modelled surface $\mathrm{CO}$ concentrations relative to observations at Mace Head and at most Northern Hemisphere stations, with a larger negative bias in winter. They hypothesised that biomass burning emissions from Russia in the latter part of the year may be too low to provide the wintertime Northern Hemisphere maximum but acknowledge that further investigations are necessary. Biases between modelled surface CO concentrations from HadGEM2-UKCA and observations are much smaller in the Southern Hemisphere and this was also the case with the G-PUCCINI and CAM-Chem model simulations (Lamarque et al., 2010).

Comparisons of vertical profiles of modelled and observed $\mathrm{CO}$ from various regions and aircraft campaigns can be found in Fig. 17. In particular, regional profiles are compared from 4 different aircraft campaigns. The Pacific Exploratory Mission in the Tropical Pacific (PEM-Tropics B) campaign took place during the wet season of the southern tropics (MarchApril 1999) and was characterized by very clean air over the South Pacific with some influence from pollution over the tropical North Pacific (Raper et al., 2001). More specifically, measurements span the latitude and longitude range of $36^{\circ} \mathrm{S}-38^{\circ} \mathrm{N}$ and $148^{\circ} \mathrm{W}-76^{\circ} \mathrm{E}$, respectively. The second aircraft campaign considered here, the Pacific Exploratory Mission West A (PEM West A) aircraft campaign, involved the NASA DC-8 aircraft, and took place in September-October 1991 over the northwestern Pacific, in which the lower tropospheric airflow was dominated by flow from mid-Pacific regions (Hoell et al., 1996). Emmons et al. (2000) split the PEM West A aircraft measurements into 5 regional profiles, namely, China Coast, Hawaii, Japan, Philippine Sea, and the western Tropical Pacific. The third set of aircraft campaign measurement data used here is from the Subsonic Assessment Ozone and Nitrogen Oxide Experiment (SONEX) and Pollution From Aircraft Emissions in the North Atlantic Flight Corridor (POLINAT 2) campaigns which took place during September to November 1997 and provides full longitudinal coverage over the Atlantic between 30 and $60^{\circ} \mathrm{N}$ (Thompson et al., 2000). In particular, the regional profiles cover three regions: Ireland, East Atlantic, and Newfoundland. Finally, the comparisons include observations from the Transport and Atmospheric Chemistry near the Equator Atlantic (TRACE A) field campaign (Fishman et al., 1996). TRACE-A took place over the tropical South Atlantic Ocean and the adjacent South American and African continents, with the specific objectives of investigating enhanced $\mathrm{O}_{3}$ concentrations over the tropical Atlantic Ocean and to quantify the role of $\mathrm{O}_{3}$ precursors associated with biomass burning in South America and southern Africa. It took place during September-October 1992 and was part of NASA's Global Tropospheric Experiment (GTE).

These aircraft measurements are not climatological in the sense that they represent long-term averages (Emmons et al., 2000). Instead, they provide snapshot distributions of trace species in different regions, at different times of the year, and are influenced by different meteorological conditions and anthropogenic and natural emissions. Therefore, given the disparities in meteorology and emissions, the comparison at best is expected to be qualitative. Some profiles compare extremely well (e.g. the West African coast from TRACE A and over the Philippine Sea during PEM-West A). Others indicate that the model is biased high (e.g. PEM-Tropics B) or low (e.g. SONEX). The high and low biases from the UKCA StdTrop chemistry scheme in HadGEM2 are not unique to this model or chemistry scheme. A comparison between an ensemble of 26 different chemistry models and CO observations from MOPITT (Shindell et al., 2006) finds that the range in modelled $\mathrm{CO}$ concentrations for the annual cycle of global mean $\mathrm{CO}$ at $500 \mathrm{hPa}$, for example, is as large as 40 ppbv. This suggests that the performance of HadGEM2UKCA relative to the regional $\mathrm{CO}$ profiles is reasonable, given the inconsistencies in meteorology and emissions.

A more direct comparison between global CO observations from the Tropospheric Emission Spectrometer (TES) instrument (Beer et al., 2001) launched on NASA's Aura satellite in 2004 and the HadGEM2-UKCA coupled configuration with the StdTrop chemistry scheme, when the model was nudged to ERA-40 reanalysis data, can be found in Sect. 6. Comparisons of modelled CO from the TropIsop chemistry scheme with TES can be found in Voulgarakis et al. (2011) and Telford et al. (2013). Surface CO comparisons of the UKCA TropIsop scheme against observations can also be found in Archibald et al. (2011). Finally, an idealised CO tracer, initialised from MOPITT, was used by Hoyle et al. (2011) to evaluate tropical deep convection in UKCA and other models. 

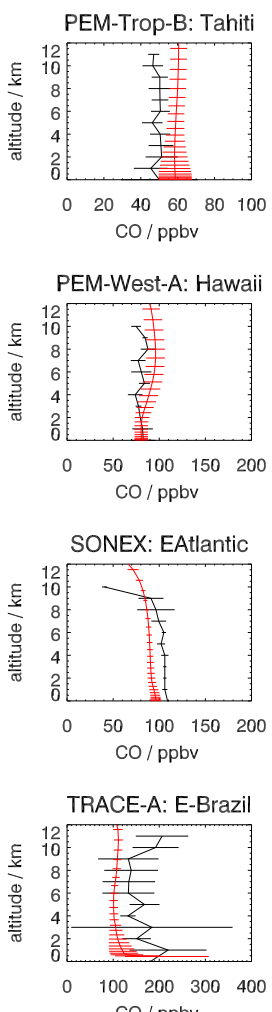

$\mathrm{CO} / \mathrm{ppbv}$
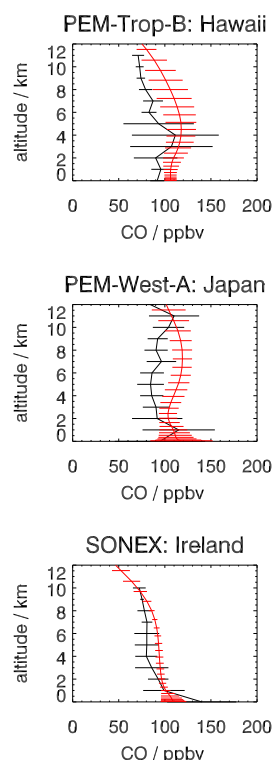

$\mathrm{CO} / \mathrm{ppbv}$

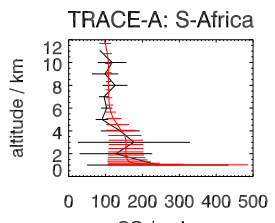

$\mathrm{CO} / \mathrm{ppbv}$
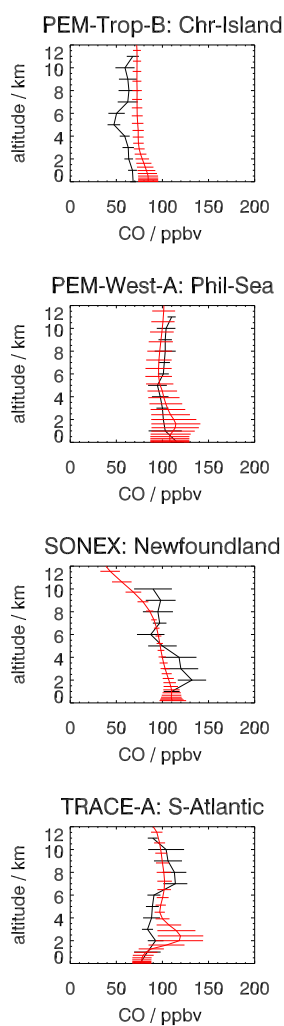
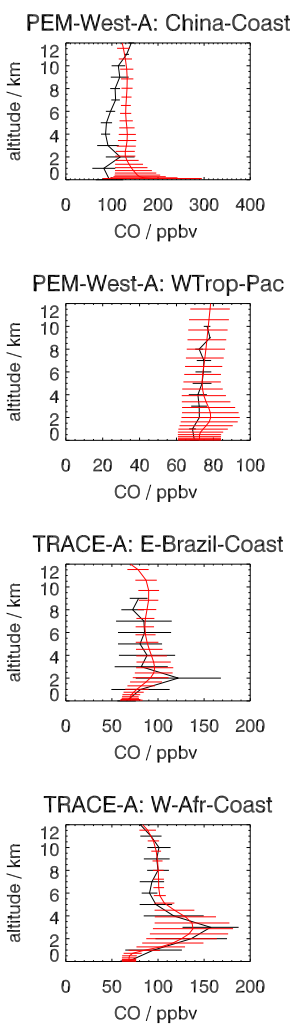

Fig. 17. Comparison between regional vertical profiles of modelled (red) and observed (black) CO from various aircraft campaigns compiled into data composites by Emmons et al. (2000). In each case, the mean concentration in each altitude bin is plotted and the horizontal lines represent the mean \pm 1 standard deviation.

\subsection{Nitrogen oxides $\left(\mathrm{NO}_{\mathrm{x}}\right)$}

Nitrogen oxides $\mathrm{NO}_{\mathrm{x}}\left(=\mathrm{NO}+\mathrm{NO}_{2}\right)$ play a critical role in tropospheric $\mathrm{O}_{3}$ production and in determining the $\mathrm{HO}_{2} / \mathrm{OH}$ ratio and $\mathrm{CH}_{4}$ lifetime against $\mathrm{OH}$ in the troposphere. Figure 18a and $\mathrm{b}$ show the modelled annual mean surface and zonal mean $\mathrm{NO}_{\mathrm{x}}$ distributions from the multi-annual simulation of the HadGEM2-UKCA StdTrop chemistry integration. Due to the conversion of $\mathrm{NO}_{\mathrm{x}}$ to $\mathrm{HNO}_{3}$ at the surface and in the lower troposphere on a timescale of 1 day, the surface plot indicates that enhanced concentrations of $\mathrm{NO}_{\mathrm{x}}$ are strongly confined to the continental source regions such as the northeastern USA, Europe, and eastern Asia. These regions are associated with anthropogenic emissions. Secondary maxima are evident over continental regions such as South America, Africa, and southern Asia, and are associated with biomass burning. The modelled global $\mathrm{NO}_{\mathrm{x}}$ distribution is also clearly influenced by ship emissions, with the main routes across the North and South Atlantic oceans, the North Pacific and the Indian Ocean. Away from the source regions, $\mathrm{NO}_{\mathrm{x}}$ concentrations are less than $10 \mathrm{pptv}$ and are consistent with other modelling studies (e.g. Lawrence et al., 1999; Jöckel et al., 2006). However, maximum concentrations can be greater than $10 \mathrm{ppbv}$ at the surface over the continental emission regions. These high concentrations are inconsistent with both observations (e.g. Horowitz et al., 1998) and other models (e.g. Hauglustaine et al., 2004), suggesting that the lifetime of surface $\mathrm{NO}_{\mathrm{x}}$ in HadGEM2-UKCA is too long due to the absence of heterogeneous chemistry (Dentener and Crutzen, 1993). Alternatively, it may be due to insufficient boundary layer mixing, as was the case at some locations with the ${ }^{222} \mathrm{Rn}$ simulations in Sect. 3.3. Sensitivity tests in which $\mathrm{NO}_{\mathrm{x}}$ emissions were added over a number of model layers reduce these maximum concentrations, bringing HadGEM2-UKCA modelled concentrations more in line with those from other models. Emissions are also handled in this way in the regional air quality configuration of UKCA (Savage et al., 2013), which was found to be important for high- $\mathrm{O}_{3}$ episodes.

The zonal annual mean distribution of $\mathrm{NO}_{\mathrm{x}}$ from the HadGEM2-UKCA StdTrop multi-annual simulation can be seen in Fig. 18b. It indicates that $\mathrm{NO}_{\mathrm{x}}$ concentrations vary by two orders of magnitude between the Northern Hemisphere boundary layer and the tropical troposphere and again, between the tropical troposphere and the stratosphere. Maximum concentrations in the annual mean are simulated in the Northern Hemisphere boundary layer between 20 and $60^{\circ} \mathrm{N}$ associated with anthropogenic emissions and in the 

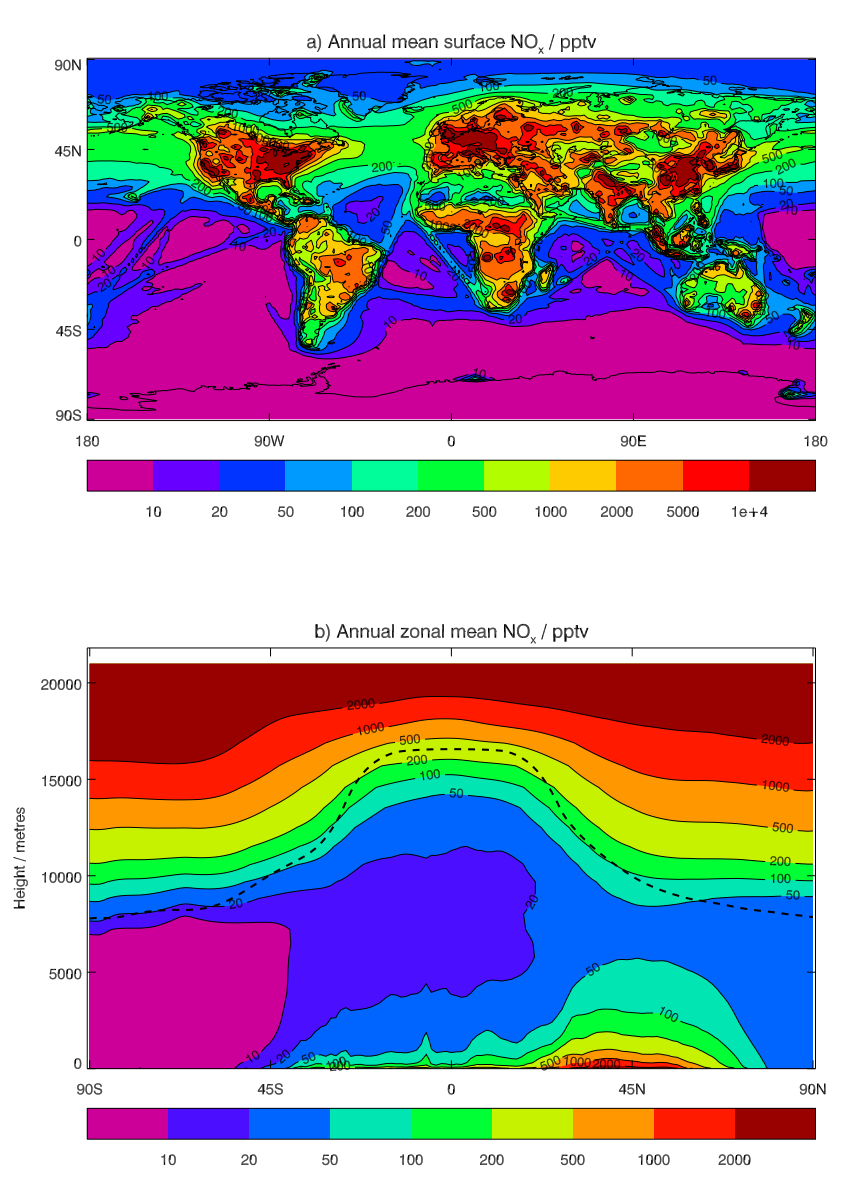

Fig. 18. Multi-annual (a) surface and (b) zonal mean $\mathrm{NO}_{\mathrm{x}}$ distributions from the StdTrop chemistry scheme in the tropospheric configuration of the UKCA model. The thick dashed line in (b) marks the position of the modelled tropopause (Hoerling et al., 1993).

stratosphere due to photolysis of $\mathrm{HNO}_{3}$. The latitudinal extent of the Northern Hemisphere boundary layer maximum varies with season and extends further poleward during the Northern Hemisphere winter. Similarly, in the annual mean, enhanced concentrations are evident in the Northern Hemisphere troposphere, the vertical extent of which varies with season and is dependent on $\mathrm{NO}_{\mathrm{x}}$ lifetime. A secondary maximum is also evident in the tropical boundary layer associated with biomass burning. The vertical extent of the enhanced concentrations in the tropics is limited to approximately $1-2 \mathrm{~km}$ depending on season, and reflects the efficient conversion of $\mathrm{NO}_{\mathrm{x}}$ to $\mathrm{HNO}_{3}$ in that region in all seasons as well as lightning emissions being too low. Nevertheless, the HadGEM2-UKCA modelled zonal mean distribution is reasonably comparable to that of other models (e.g. Collins et al., 1997; Lawrence et al., 1999). One difference between the models is related to the latitudinal extent of the enhanced concentrations in the Northern Hemisphere. In both HadGEM2-UKCA and the MATCH-MPIC (Lawrence et al., 1999) models, concentrations of 100 pptv only extend up to $70^{\circ} \mathrm{N}$, whereas in the STOCHEM (Collins et al., 1997) model, simulated concentrations poleward of $70^{\circ} \mathrm{N}$ are of the order of $1 \mathrm{ppbv}$ during the Northern Hemisphere winter.

Comparisons of modelled $\mathrm{NO}_{\mathrm{x}}$ concentrations with regional observations derived from a variety of aircraft campaigns (Emmons et al., 2000) can be found in Fig. 19. Given the short lifetime of $\mathrm{NO}_{\mathrm{x}}$, it does not provide the integrated view of regional emissions and transport that $\mathrm{CO}$ provides (Shindell et al., 2006). Nevertheless, it does give some indication of the $\mathrm{NO}_{\mathrm{x}}$ emissions being used in the model. There is considerable variability in the observed concentrations in both time and space. However, in some locations (e.g. East Brazil Coast during TRACE A), the model captures the observed "C-shaped" profiles, with high concentrations in the polluted boundary layer, decreasing concentrations in the lowest troposphere, and enhanced concentrations aloft due to both lightning emissions and stratospheric input. In other locations, however, the HadGEM2-UKCA model fails to capture the high concentrations in the mid and upper troposphere. Part of this bias may be related to lightning emissions being too low in the model (Young et al., 2013), although it may also be related to the emissions being added to the lowest model layer, insufficient boundary layer mixing and/or lack of convective uplift. The comparison shows that this aspect of the model performs satisfactorily but that ventilation of the boundary through mixing and/or convection may need improving.

The aircraft observations from Emmons et al. (2000) were also used for comparisons of $\mathrm{HNO}_{3}$ and PAN; these are shown in Figs. 20 and 21, respectively. In the case of PAN, apart from a few locations (e.g. China Coast during PEM West A), the model consistently underestimates PAN concentrations by up to a factor of 5 . This is not unexpected, given the lack of treatment of NMVOCs in the StdTrop chemistry scheme. In the case of $\mathrm{HNO}_{3}$, there are some campaigns and locations where modelled and observations concentrations match very well (e.g. Ireland and Newfoundland during SONEX, East Brazil and East Brazil Coast during TRACEA. However, there are other locations and campaigns where modelled $\mathrm{HNO}_{3}$ is biased low (e.g. Christmas Island during PEM-Tropics B) or high (e.g. China Coast during PEM West A). Apart from the mismatch between the meteorology of the model and that of the campaigns, previous work has highlighted the difficulty of simulating $\mathrm{HNO}_{3}$ (e.g. Bey et. al., 2001). As well as being sensitive to the washout parameterization and precipitation patterns and intensity, the lack of conversion from gas phase $\mathrm{HNO}_{3}$ to nitrate aerosol and the lack of heterogeneous processes in this model configuration are potential reasons for the model-observations discrepancies. Likewise, the lack of PAN formation from the StdTrop chemistry scheme will have implications for the $\mathrm{NO}_{\mathrm{y}}$ partitioning and $\mathrm{HNO}_{3}$ distributions. The impact of including isoprene (TropIsop chemistry scheme) on $\mathrm{NO}_{\mathrm{y}}$ partitioning and other aspects of the tropospheric chemistry performance will be discussed further in Sect. 6 . 

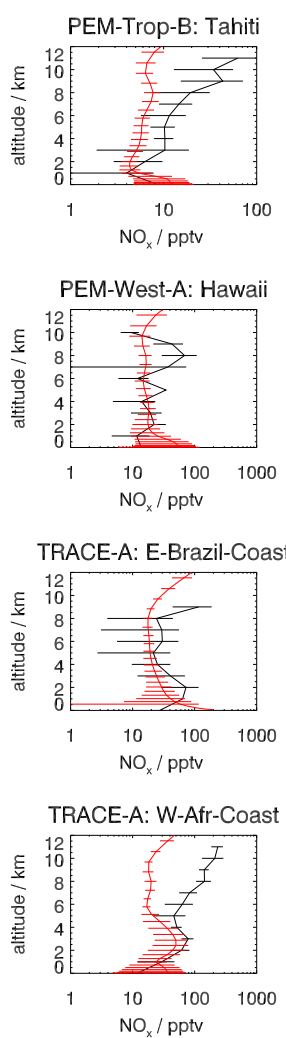
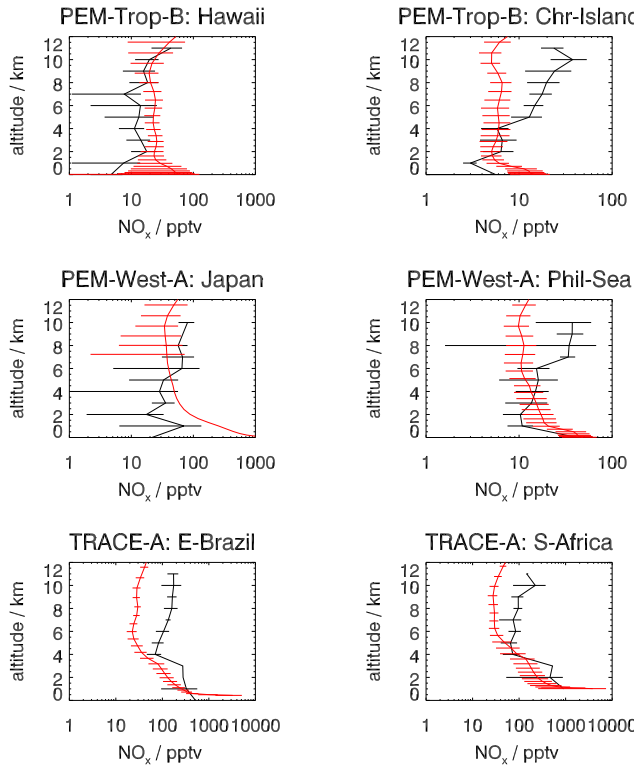
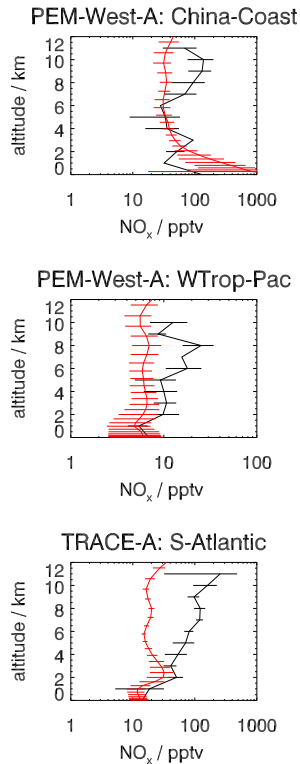

Fig. 19. Comparison between regional vertical profiles of modelled (red) and observed (black) $\mathrm{NO}_{\mathrm{x}}$ from various aircraft campaigns compiled into data composites by Emmons et al. (2000). In each case, the mean concentration in each altitude bin is plotted and the horizontal lines represent the mean \pm 1 standard deviation.

\subsection{Hydroxyl radical $(\mathrm{OH})$}

The zonal and diurnal mean distribution of $\mathrm{OH}$ for January and July from the UKCA StdTrop chemistry scheme coupled to HadGEM2 can be found in Fig. 22. The latitudinal and seasonal variations in $\mathrm{OH}$ can be attributed to the distributions of ultraviolet (UV) radiation and water vapour which together provide the primary source of $\mathrm{OH}$ through the $\mathrm{O}(1 \mathrm{D})+\mathrm{H}_{2} \mathrm{O}$ reaction (Levy, 1971). Concentrations are typically higher during the summer in the Northern Hemisphere, reflecting the role of $\mathrm{O}_{3}$ and $\mathrm{NO}_{\mathrm{x}}$ in enhancing $\mathrm{OH}$ production. Maximum $\mathrm{OH}$ concentrations are found in the boundary layer, as is the case in the ECHAM/MESSy1 model (Jöckel et al., 2006) and was attributed by them to a lack of liquidphase $\mathrm{OH}$ chemistry, $\mathrm{HO}_{\mathrm{x}}$ uptake by aerosols, and secondary organic aerosol (SOA) formation in the boundary layer. In the StdTrop chemistry configuration of UKCA, there is also a lack of NMVOC chemistry, which is known to reduce $\mathrm{OH}$ in the boundary layer, particularly over forested continents (Houweling et al., 1998; Wang et al., 1998; Roelofs and Lelieveld, 2000). Minimum concentrations are modelled in the winter mid and high latitudes as well as in the summer UTLS.
The global annual mean $\mathrm{OH}$ concentration from the HadGEM2-UKCA StdTrop simulation is $0.8 \pm 0.03 \times 10^{6}$ molecules $\mathrm{cm}^{-3}$ and $1.03 \pm 0.03 \times 10^{6}$ molecules $\mathrm{cm}^{-3}$ based on weighting factors of air mass and reaction rate with $\mathrm{CH}_{4}$, respectively (Lawrence et al., 2001). In both cases, the standard deviation represents the variability in the global mean concentration using different vertical domains (i.e. region below modelled climatological tropopause, region below $100 \mathrm{hPa}$, region below $200 \mathrm{hPa}$, and region where $\mathrm{O}_{3}$ is less than or equal to $100 \mathrm{ppbv}$ ), confirming the weak dependence of the global mean $\mathrm{OH}$ concentration on the choice of domain for these particular weightings (Lawrence et al., 2001). The air mass weighted $\mathrm{OH}$ concentration from StdTrop is, however, on the low side of the chemical models that participated in the recent Atmospheric Chemistry and Climate Model Intercomparison Project (ACCMIP; Lamarque et al., 2013), in which the multi-model mean concentration is $1.11 \pm 0.18 \times 10^{6}$ molecules $\mathrm{cm}^{-3}$ (Voulgarakis et al., 2013). This low bias in $\mathrm{OH}$ is partly due to the known negative bias in $J\left(\mathrm{O}^{1} \mathrm{D}\right)$ rates from the offline photolysis scheme (Telford et al., 2013). Lightning emissions of $\mathrm{NO}_{\mathrm{x}}$ were also inadvertently too low (Young et al., 2013), contributing to reduced efficiency of $\mathrm{OH}$ recycling in these model 

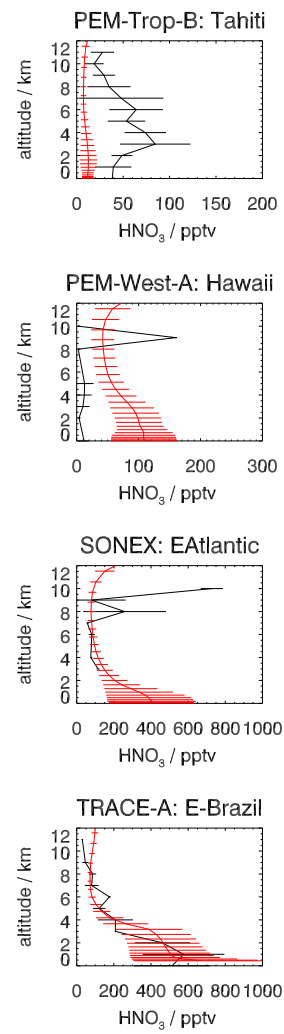
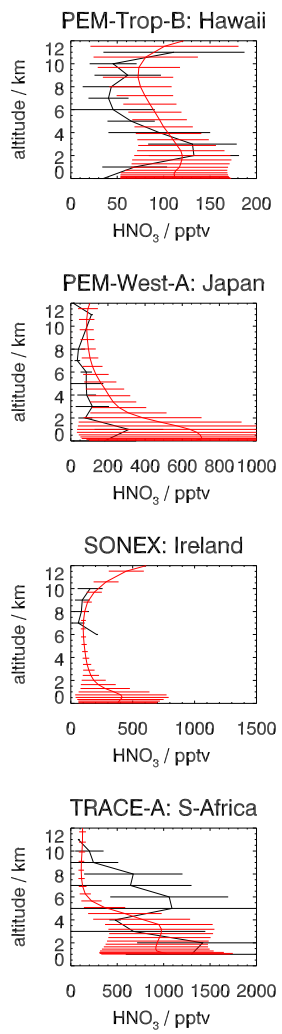
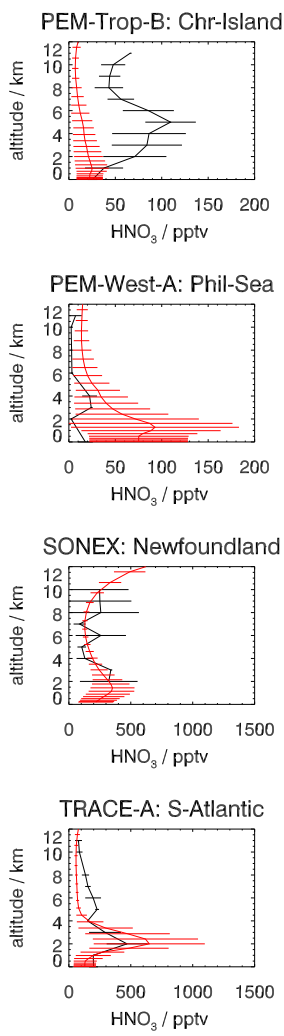
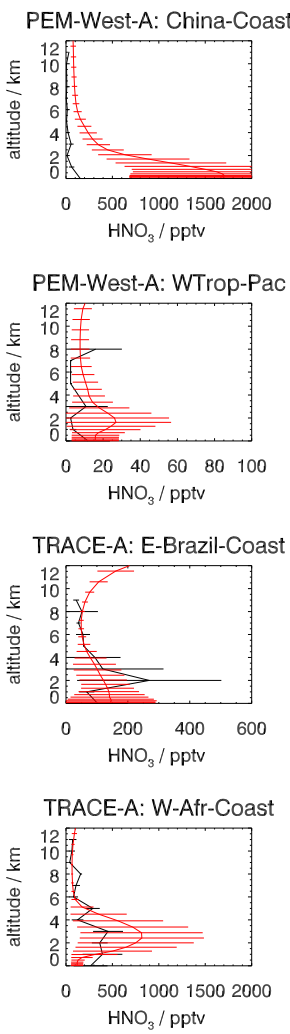

Fig. 20. Comparison between regional vertical profiles of modelled (red) and observed (black) $\mathrm{HNO}_{3}$ from various aircraft campaigns compiled into data composites by Emmons et al. (2000). In each case, the mean concentration in each altitude bin is plotted and the horizontal lines represent the mean \pm 1 standard deviation.

runs. As indicated in Sect. 5.1, the atmospheric lifetime associated with the tropospheric $\mathrm{CH}_{4}$ burden and its loss by $\mathrm{OH}$, calculated following Lawrence et al. (2001), is $9.96 \mathrm{yr}$, which is in excellent agreement with the estimates from Prather et al. (2001), Stevenson et al. (2006) and the multi-model mean estimate from Voulgarakis et al. (2013). Note that Voulgarakis et al. (2013) quote the lifetime for the full atmosphere, rather than the tropospheric values used here.

The impact of including isoprene in the UKCA chemistry scheme (i.e. TropIsop) on global mean $\mathrm{OH}$ is discussed in Sect. 12. Comparisons of modelled $\mathrm{OH}$ from the UKCA TropIsop chemistry scheme against surface measurements from a southeast Asian tropical forest can also be found in Archibald et al. (2011).

\subsection{Ozone $\left(\mathrm{O}_{3}\right)$}

In Fig. 23a and b, the HadGEM2-UKCA modelled global distribution of surface $\mathrm{O}_{3}$ concentrations for January and July are shown, respectively. In January, the HadGEM2UKCA model simulates maximum surface concentrations over central Africa (greater than $60 \mathrm{ppbv}$ ) and south Asia (50-60 ppbv) associated with $\mathrm{O}_{3}$ precursor emissions from biomass burning and industrial sources, respectively. High surface concentrations are also simulated over the north Pacific (typically over $40 \mathrm{ppbv}$ ) and Atlantic (35-40 ppbv) Oceans, providing evidence for the longer $\mathrm{O}_{3}$ lifetime and transport from the stratosphere during the Northern Hemisphere winter. The calculated stratospheric ozone contribution to the modelled surface $\mathrm{O}_{3}$ abundance in the model is of the order of 40-50\% in these regions in January (not shown) and is similar to that calculated by Sudo et al. (2002). By contrast, concentrations are less than $20 \mathrm{ppbv}$ over the US and the Eurasian continental regions. At the low $\mathrm{HO}_{\mathrm{x}}$ concentrations found in the Northern Hemisphere winter, $\mathrm{O}_{3}$ production is slow and in the boundary layer, $\mathrm{O}_{3}$ is removed directly through titration by NO. Nevertheless, the causes of this low bias will be investigated further in Sect. 6. In the Southern Hemisphere, higher surface concentrations are found over the continental regions (typically 20-30 ppbv) than over the remote oceans (less than 20 ppbv). In July, $\mathrm{O}_{3}$ concentrations are generally much higher than in January, with maximum concentrations over southern Europe, the Middle East, central Asia, and the continental US. High concentrations are also evident over western Africa associated with biomass burning and there is some evidence of outflow from the continental regions to the oceanic regions. However, apart from 

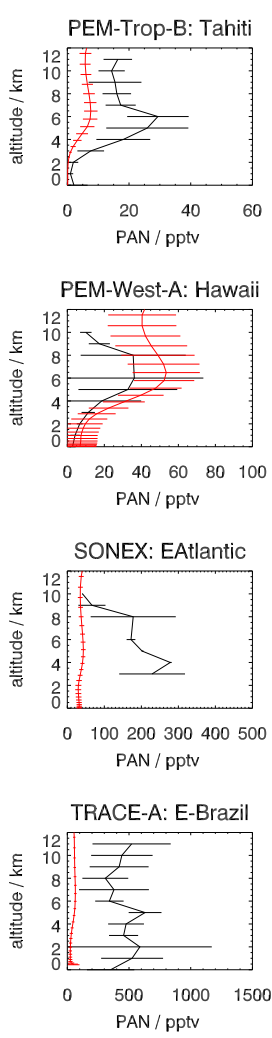
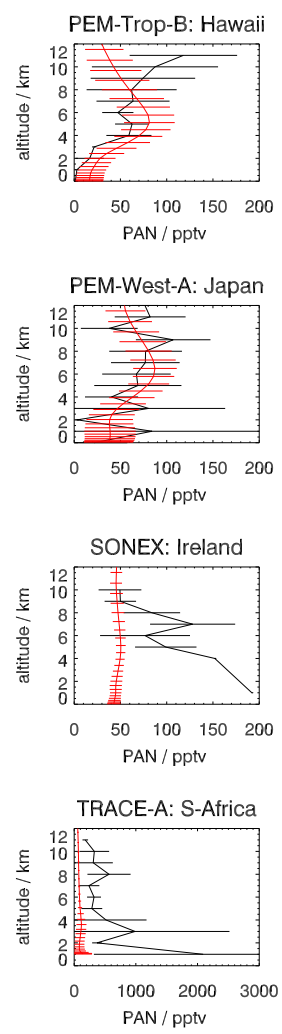
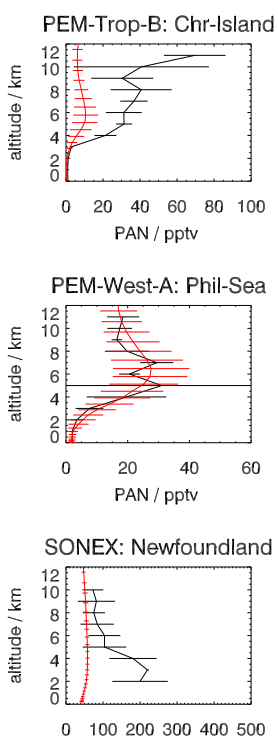

PAN / pptv

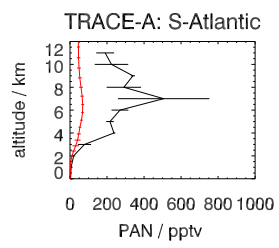

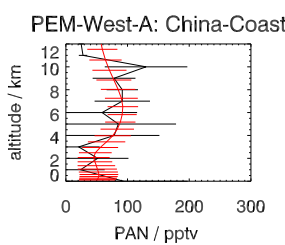
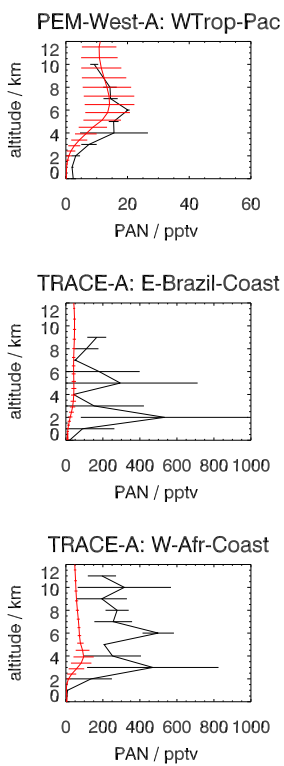

Fig. 21. Comparison between regional vertical profiles of modelled (red) and observed (black) PAN from various aircraft campaigns compiled into data composites by Emmons et al. (2000). In each case, the mean concentration in each altitude bin is plotted and the horizontal lines represent the mean \pm 1 standard deviation.

the low bias in Northern Hemisphere mid-latitude continental regions, the main features of the surface distribution, both in January and July, is consistent with other modelling studies (e.g. Hauglustaine et al., 2004; Zeng et al., 2008).

The multi-annual zonal mean $\mathrm{O}_{3}$ distribution for January and July can be found in Fig. 24. It indicates that tropospheric $\mathrm{O}_{3}$ concentrations typically increase with height and are higher in the Northern Hemisphere than in the Southern Hemisphere in both seasons. Northern Hemisphere concentrations in the mid-troposphere are typically $40-60 \mathrm{ppbv}$, whereas Southern Hemisphere concentrations are closer to 30-40 ppbv. Maximum concentrations are found in the upper troposphere of the Northern Hemisphere during July (greater than $60 \mathrm{ppbv}$ ) with minimum concentrations near the surface in the Southern Hemisphere in January. In the tropics, there is evidence of a secondary minimum in the mid- and upper troposphere due to advection and convective transport of $\mathrm{O}_{3}$ poor air from the surface. The influence of the stratosphere on the tropospheric concentrations is also clearly evident in both hemispheres, due to isentropic transport of high- $\mathrm{O}_{3}$ air across the sub-tropical jetstream. In the Northern Hemisphere mid-troposphere $\left(45^{\circ} \mathrm{N}, 5 \mathrm{~km}\right)$, stratospheric $\mathrm{O}_{3}$ contributes $50-60 \%$ to the modelled tropospheric $\mathrm{O}_{3}$ distribution in January which reduces to $35-40 \%$ in July. In the
Southern Hemisphere, on the other hand, stratospheric $\mathrm{O}_{3}$ contributes $40-50 \%$ and $50-60 \%$ to the mid-troposphere modelled concentrations in January and July, respectively, and is similar to that of other models (e.g. Hauglustaine et al., 2004). A comparison of the zonal annual mean with the ACCENT (Atmospheric Composition Change: the European Network of excellence) multi-model ensemble by Stevenson et al. (2006) indicates that modelled $\mathrm{O}_{3}$ from HadGEM2UKCA sits within \pm 1 standard deviation of the ensemble mean in most regions and altitudes. The most notable exception is in the tropical mid- and upper troposphere, where the absence of an isoprene mechanism in StdTrop may account for some of the discrepancy (Pöschl et al., 2000; von Kuhlmann et al., 2004). However, the role of lightning emissions cannot be ruled out and is explored later in this section.

The chemistry scheme being evaluated here, due to its lack of NMVOC chemistry, is not expected to reproduce observed surface $\mathrm{O}_{3}$ in regions influenced by NMVOC emissions from vegetation and industry. Therefore, a comparison between modelled and observed surface concentrations of $\mathrm{O}_{3}$ (Fig. 25) is indicative only and includes the statistical measures of skill used in the previous surface comparisons. The model captures both the seasonal cycle and the absolute concentrations of surface $\mathrm{O}_{3}$ at northern mid-latitudes (e.g. Mace 

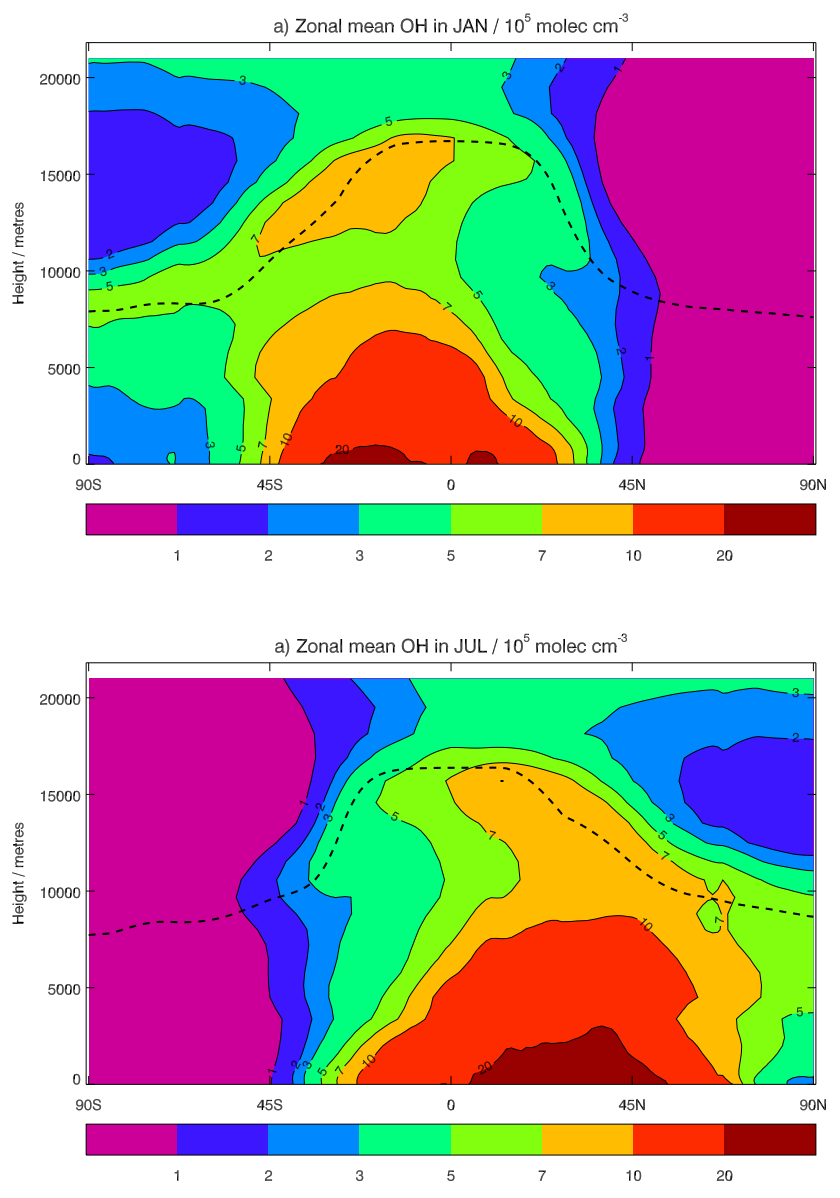

Fig. 22. Multi-annual zonal and diurnal mean $\mathrm{OH}$ distributions from the StdTrop chemistry scheme in the tropospheric configuration of the UKCA model in (a) January and (b) July. The thick dashed lines mark the position of the modelled tropopause (Hoerling et al., 1993).

Head has a relative annual mean bias of $-1.7 \%$, a root mean square error of $2.3 \mathrm{ppbv}$, a correlation coefficient of 0.84 , and a model score of $100 \%$ ). The springtime maximum at the remote sites of Mace Head and Bermuda is represented very well along with the summertime maximum at the polluted site of Niwot Ridge. In the tropics, the seasonal cycle of the surface observations is reproduced well (Mauna Loa, Barbados, and Samoa) but the model overestimates concentrations by up to $10 \mathrm{ppbv}$. A similar disagreement in the tropics between modelled and observed surface concentrations was also evident in the LMDz-INCA model (Hauglustaine et al., 2004). The model performs less well at the northern high latitude site of Barrow (the relative annual mean bias and correlation coefficient are $-19.5 \%$ and -0.65 , respectively). Although the summertime concentrations are captured reasonably well, the model fails to reproduce the autumn-winter maximum; modelled concentrations are underestimated by $50 \%$. The cause of this discrepancy is unclear but modelled surface ${ }^{210} \mathrm{~Pb}$ concentrations at Barrow in Sect. 3.4 suggest
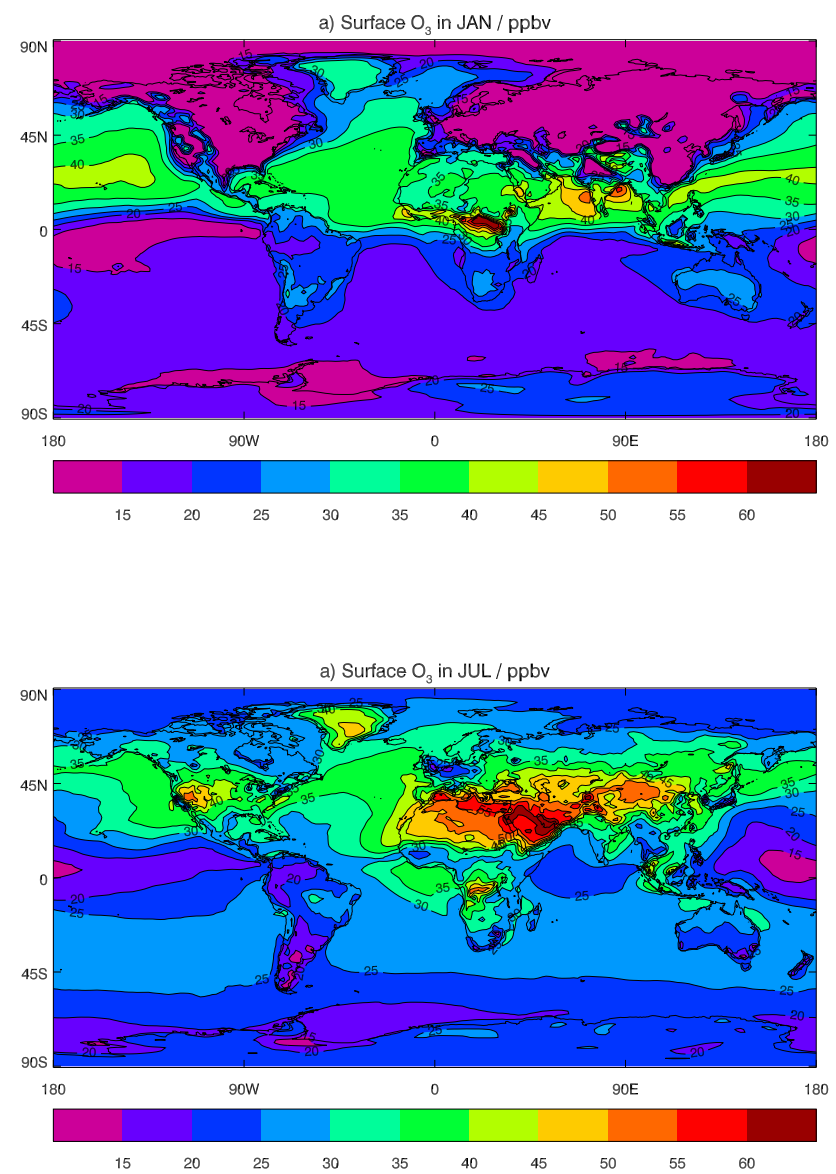

Fig. 23. Multi-annual monthly mean distribution of surface $\mathrm{O}_{3}$ from the StdTrop chemistry scheme in the tropospheric configuration of the UKCA model in (a) January and (b) July.

that the model is representing the long-range boundary layer transport into the Arctic well. Other possibilities for the discrepancy could be biases in $\mathrm{O}_{3}$-precursor emissions (as seen in $\mathrm{CO}$ in Sect. 5.2), lack of gas-phase and heterogeneous bromine chemistry (e.g. Yang et al., 2005), excessive titration of $\mathrm{O}_{3}$ by $\mathrm{NO}$, in addition to the difficulty that a coarse-grid model has in representing the uniqueness of the observational site at Barrow.

The radiative forcing efficiency on a per molecule basis is greatest for $\mathrm{O}_{3}$ changes near the tropopause (Lacis et al., 1990), thus making upper levels more important than the surface for climate forcing by $\mathrm{O}_{3}$. As a result, in Fig. 26, a comparison between HadGEM2-UKCA and ozonesonde observations (Logan et al., 1999; Thompson et al., 2003a, b) is presented. Also included in the comparison is the ACCENT multi-model ensemble from Stevenson et al. (2006). HadGEM2-UKCA performs exceptionally well in the northern extra-tropics at all pressure levels in capturing both the seasonal cycle and the absolute $\mathrm{O}_{3}$ concentrations. In particular, the ACCENT ensemble underestimates the amplitude of the seasonal cycle and overestimates Northern Hemisphere 

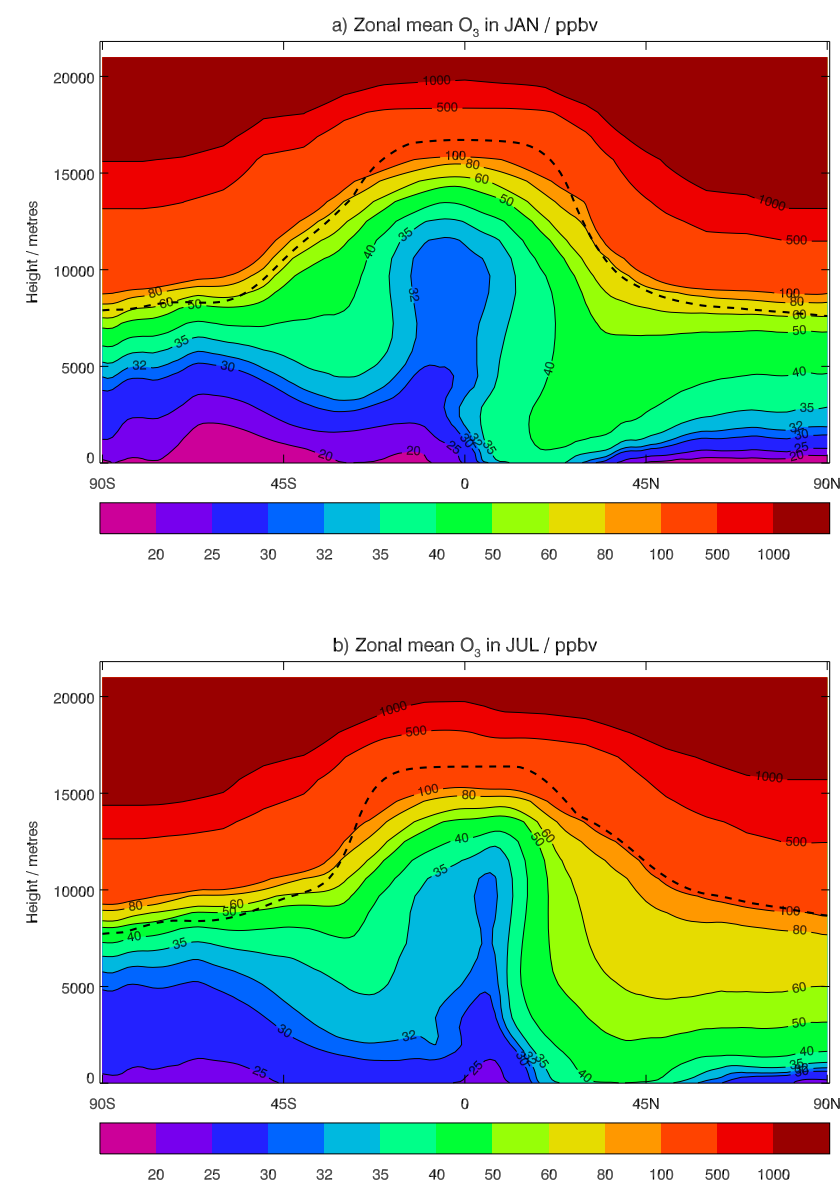

Fig. 24. Multi-annual zonal and diurnal mean $\mathrm{O}_{3}$ distributions from the StdTrop chemistry scheme in the tropospheric configuration of the UKCA model in (a) January and (b) July. The thick dashed lines mark the position of the modelled tropopause (Hoerling et al., 1993).

wintertime $\mathrm{O}_{3}$ by approximately $10 \mathrm{ppbv}$ (Stevenson et al., 2006), whereas HadGEM2-UKCA reproduces both the amplitude of the seasonal cycle and the observed wintertime $\mathrm{O}_{3}$ at 750 and $500 \mathrm{hPa}$ very well - the root mean square error (RMSE) between UKCA and the observations is 1.1 and $2.2 \mathrm{ppbv}$, respectively (cf. RMSEs of 4.4 and $6.7 \mathrm{ppbv}$, respectively, for the ACCENT multi-model mean). Similarly, the comparison of HadGEM2-UKCA with observations at 850,600 , and $400 \mathrm{hPa}$ in the northern extra-tropics is very good (RMSEs of 1.6, 1.6, and $3.9 \mathrm{ppbv}$, respectively). The model, however, tends to underestimate the observed $\mathrm{O}_{3}$ concentrations at $250 \mathrm{hPa}$ with a slight overestimate at $100 \mathrm{hPa}$. This overestimate at $100 \mathrm{hPa}$ is also evident in the tropics and results in a warming of the tropical tropopause and a moistening of the stratosphere when $\mathrm{O}_{3}$ from UKCA is passed to the climate model's radiation scheme in place of a prescribed $\mathrm{O}_{3}$ climatology (O'Connor et al., 2009). An underestimate of modelled $\mathrm{O}_{3}$ from HadGEM2-UKCA at $250 \mathrm{hPa}$ in comparison with observations is also evident in the tropics and places
UKCA at the lower end of the ACCENT multi-model ensemble. At $500 \mathrm{hPa}$, HadGEM2-UKCA is again at the lower end of the model ensemble and is biased low in comparison with the observations in the $0-30^{\circ} \mathrm{S}$ latitude band. At the same pressure surface in the northern tropics, however, HadGEM2-UKCA is in better agreement with the observations (RMSE is $4.3 \mathrm{ppbv}$ ) than the ensemble mean (RMSE is $7.9 \mathrm{ppbv}$ ) but the number of observational sites (4) covering this region is limited. In the southern extra-tropics, HadGEM2-UKCA matches the observations very well at 850 and $750 \mathrm{hPa}$, with also a good agreement at $750 \mathrm{hPa}$ with the ensemble mean. Aloft, HadGEM2-UKCA performs well in comparison with both the observations and the multimodel ensemble but tends to underestimate $\mathrm{O}_{3}$ during the Southern Hemisphere winter. The model intercomparison by Stevenson et al. (2006) indicated that the greatest variability in modelled $\mathrm{O}_{3}$ is in the tropics as a result of model differences in the treatments of convection, lightning $\mathrm{NO}_{\mathrm{x}}$ emissions, biogenic isoprene emissions and biomass burning emissions. Indeed, it was found that the low bias from HadGEM2-UKCA relative to observations and the other ACCENT models was due to the lightning $\mathrm{NO}_{\mathrm{x}}$ emissions in the model being inadvertently lower (i.e. $<2 \mathrm{TgN} \mathrm{yr}^{-1}$ ) than the anticipated level of $5 \mathrm{Tg} \mathrm{N} \mathrm{yr}^{-1}$ (Young et al., 2013). A sensitivity study in which the lightning emissions were increased results in a reduction in the HadGEM2-UKCA tropical $\mathrm{O}_{3}$ bias. Stevenson et al. (2006) also noted that the midlatitudes are less affected by convection, lightning, biomass burning and biogenic emissions and the performance of HadGEM2-UKCA is indeed better in the extra-tropics than in the tropics. In terms of the overall performance of the model, a RMSE for HadGEM2-UKCA was calculated using modelled and observed monthly mean $\mathrm{O}_{3}$ concentrations at 750,500 , and $250 \mathrm{hPa}$ and excluding the extra-tropics at $250 \mathrm{hPa}$; HadGEM2-UKCA has a RMSE of $7.7 \mathrm{ppbv}$, well within the range of RMSEs across the ACCENT models of 5.1-18.0 ppbv (Stevenson et al., 2006). For a comparison of the more recent ACCMIP multi-model ensemble, including UKCA StdTrop in HadGEM2, with the ACCENT models (Stevenson et al., 2006) and ozonesonde and satellite observations, the reader is referred to Young et al. (2013).

An additional comparison, making use of the Fortuin and Kelder (1998) $\mathrm{O}_{3}$ climatology which is based on ozonesonde and satellite measurements made between 1980 and 1991, is shown in Fig. 27. This observational climatology was used to evaluate some of the models which participated in the ACCENT model intercomparison (Gauss et al., 2006). In the tropical troposphere $\left(20^{\circ} \mathrm{S}-20^{\circ} \mathrm{N}, 0-12 \mathrm{~km}\right)$, they found that all models except one agreed with the observed $\mathrm{O}_{3}$ climatology to within $\pm 40 \%$. This is also the case with HadGEM2UKCA, with maximum deviations occurring in the $8-12 \mathrm{~km}$ altitude range. The study by Gauss et al. (2006) also indicated that near-surface $\mathrm{O}_{3}$ at $30^{\circ} \mathrm{N}$ in the ACCENT models was higher than the climatology and extended to other latitudes and higher altitudes to varying degrees. Modelled 
Table 12. Additional sensitivity experiments with the HadGEM2UKCA coupled model.

\begin{tabular}{cl}
\hline Expt. No. & Change relative to control \\
\hline 1 & Fast-J on \\
2 & Nudged to ERA-40 re-analyses \\
3 & Prescribed deposition velocities \\
4 & Surface emissions added to bottom 4 model levels \\
5 & Increase in emissions of NMVOCs in StdTrop \\
6 & TropIsop on \\
\hline
\end{tabular}

$\mathrm{O}_{3}$ from HadGEM2-UKCA also shows a positive anomaly in comparison with the Fortuin and Kelder (1998) climatology at the surface between 15 and $30^{\circ} \mathrm{N}$ which extends upwards to $3 \mathrm{~km}$ at $25^{\circ} \mathrm{N}$. Thirdly, for the Northern Hemisphere extra-tropical troposphere $\left(45-90^{\circ} \mathrm{N}, 0-8 \mathrm{~km}\right), \mathrm{O}_{3}$ was simulated to within $\pm 30 \%$ by two of the ACCENT models and to within $\pm 10-20 \%$ by the other models. HadGEM2-UKCA appears to be in better agreement with the climatology in this region than in the tropics, with anomalies generally less than $20 \%$. The most notable exceptions to this are negative anomalies in excess of $25 \%$ near the surface between 45 and $75^{\circ} \mathrm{N}$ and in the upper troposphere/lower stratosphere. The former discrepancy could be related to the lack of NMVOC chemistry in StdTrop and will be explored in Sect. 6, whereas the latter discrepancy can be most likely attributed to a mismatch in tropopause location between HadGEM2-UKCA and the $\mathrm{O}_{3}$ climatology. Gauss et al. (2006) argue that an agreement between modelled and observed $\mathrm{O}_{3}$ concentrations of better than 20-40\% cannot be expected due to the mismatch in meteorology between the observations and that used by the models, the time lag between the model simulations and the observations, and the lack of interannual variability in the models' prescribed emissions. From a UKCA perspective, although these caveats also apply, the key point to note is that the performance of HadGEM2-UKCA relative to the climatology is quantitatively comparable to that of the other models.

Furthermore, a more direct comparison between global $\mathrm{O}_{3}$ observations from the TES instrument on NASA's Aura satellite and the HadGEM2-UKCA coupled configuration with the StdTrop chemistry scheme, when the model has been nudged to year-specific meteorology, can be found in Sect. 6 .

A tropospheric $\mathrm{O}_{3}$ budget has been calculated from the 10 -yr simulation, using the combined isentropic-dynamical definition for the tropopause (Hoerling et al., 1993). The chemical production and loss terms for the UKCA StdTrop chemistry scheme coupled to HadGEM2 and with offline photolysis are $3064 \pm 18$ and $2735 \pm 19 \mathrm{Tg} \mathrm{O}_{3} \mathrm{yr}^{-1}$, respectively, with the troposphere being a net chemical source of $\mathrm{O}_{3}$. Dry deposition accounts for $931 \pm 4 \mathrm{Tg} \mathrm{O}_{3} \mathrm{yr}^{-1}$ and the inferred strength of the stratosphere-troposphere exchange (STE) term is $603 \pm 11 \mathrm{Tg} \mathrm{O}_{3} \mathrm{yr}^{-1}$. The chemical production term is low relative to the ACCENT ensemble (cf. $5110 \pm 606 \mathrm{Tg} \mathrm{O}_{3} \mathrm{yr}^{-1}$ from Stevenson et al., 2006). However, it is consistent with chemistry schemes in which there is no treatment of isoprene (cf. $3358 \pm 647 \mathrm{Tg} \mathrm{O}_{3} \mathrm{yr}^{-1}$ from Wild, 2007). The deposition and STE terms, on the other hand, compare well with other estimates. STE from the ACCENT models, for example, was $552 \pm 168 \mathrm{Tg} \mathrm{O}_{3} \mathrm{yr}^{-1}$ (Stevenson et al., 2006) and the estimate here lies within $10 \%$ of the ACCENT multi-model mean and the observational constraint of $550 \pm 140 \mathrm{Tg} \mathrm{O}_{3} \mathrm{yr}^{-1}$ from Olsen et al. (2001). Dry deposition, likewise, matches estimates from ACCENT (cf. $1003 \pm 200 \mathrm{Tg} \mathrm{O}_{3} \mathrm{yr}^{-1}$ from Stevenson et al. (2006)) and other model estimates (cf. $902 \pm 255 \mathrm{Tg} \mathrm{O}_{3} \mathrm{yr}^{-1}$ from Wild, 2007).

\section{Sensitivity experiments}

As indicated in previous sections, a number of sensitivity experiments were carried out. The control experiment was that which was evaluated above and in each of the sensitivity experiments, a change relative to the control was introduced; these are listed in Table 12. In all cases, $\mathrm{O}_{3}$ and $\mathrm{CH}_{4}$ did not influence the model dynamics and hence, the meteorology of the sensitivity experiments was identical to that of the control. The only exception to this is Expt. No. 2, in which the meteorology was nudged towards ERA-40 re-analyses; this experiment will be discussed further in the context of TES $\mathrm{CO}$ and $\mathrm{O}_{3}$ comparisons below.

The first of the sensitivity experiments (Expt. No. 1) involved a switch from the offline photolysis scheme to the online Fast-J scheme (Wild et al., 2000). This had the effect of reducing zonal mean $\mathrm{O}_{3}$ concentrations everywhere, with the maximum reduction of the order of $20 \%$ occurring in the tropical and Southern Hemisphere lower troposphere where $\mathrm{O}_{3}$ concentrations themselves are at a minimum. In particular, Southern Hemisphere concentrations were reduced by $5-20 \%$, whereas in the Northern Hemisphere, $\mathrm{O}_{3}$ was reduced by typically less than $5 \%$. Fast-J also resulted in changes in the terms of the tropospheric $\mathrm{O}_{3}$ budget as follows: chemical production increased by $19 \%$, chemical loss increased by $25 \%$, STE increased by $5 \%$, and the dry deposition term decreased by $7 \%$. These changes are consistent with comparisons using the TropIsop chemistry scheme between the offline scheme and Fast-JX (cf. +23, +29, +7, and $-3 \%$ changes for chemical production, chemical loss, STE, and dry deposition, respectively, from Telford et al., 2013). Despite the increase in the chemical production term here, its magnitude is still biased low by $28 \%$ relative to the ACCENT multi-model mean (Stevenson et al., 2006) and reflects the sensitivity of gross chemical production of $\mathrm{O}_{3}$ to isoprene (Wild, 2007). In comparison with observations, Fast-J resulted in marginal improvements in modelled surface $\mathrm{O}_{3}$ at Barbados and Samoa (the RMSEs were reduced to 3.6 and $2.9 \mathrm{ppbv}$, respectively) but little change in the 

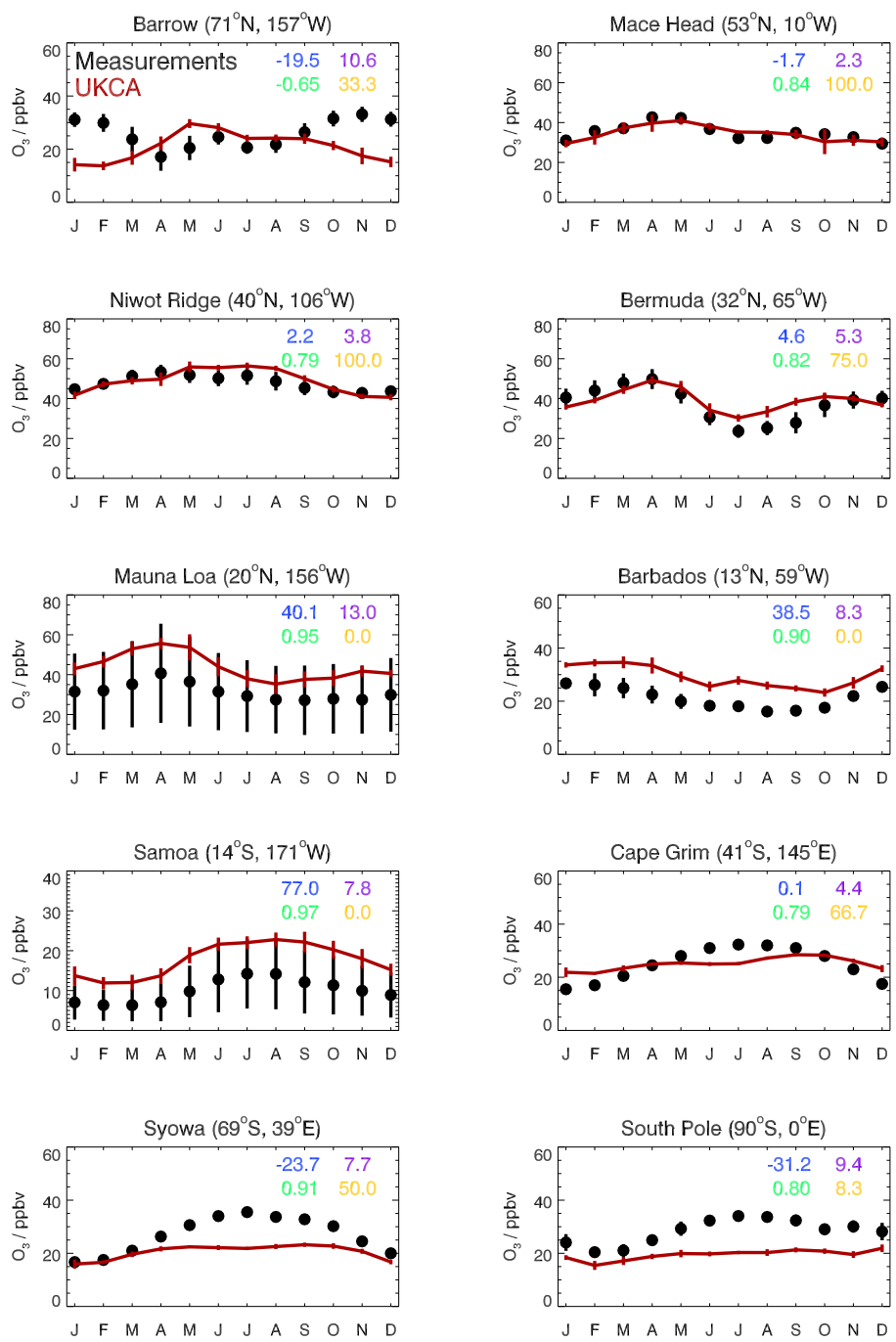

Fig. 25. Comparison between multi-annual monthly mean modelled surface $\mathrm{O}_{3}$ concentrations with surface observations. The surface sites include Barrow $\left(71^{\circ} \mathrm{N}, 157^{\circ} \mathrm{W}\right)$, Mace Head $\left(53^{\circ} \mathrm{N}, 10^{\circ} \mathrm{W}\right)$, Niwot Ridge $\left(40^{\circ} \mathrm{N}, 106^{\circ} \mathrm{W}\right)$, Bermuda $\left(32^{\circ} \mathrm{N}, 65^{\circ} \mathrm{W}\right)$, Mauna Loa $\left(20^{\circ} \mathrm{N}\right.$, $\left.156^{\circ} \mathrm{W}\right)$, Barbados $\left(13^{\circ} \mathrm{N}, 59^{\circ} \mathrm{W}\right)$, Samoa $\left(14^{\circ} \mathrm{S}, 171^{\circ} \mathrm{W}\right)$, Cape Grim $\left(41^{\circ} \mathrm{S}, 145^{\circ} \mathrm{E}\right)$, Syowa $\left(69^{\circ} \mathrm{S}, 39^{\circ} \mathrm{E}\right)$, and the South Pole $\left(90^{\circ} \mathrm{S}\right.$, $0^{\circ} \mathrm{E}$ ). The relative annual mean bias (in \%), the root mean square error (in ppbv), the correlation coefficient, and the model score (the percentage of months when the modelled and observed monthly mean concentrations are within $20 \%$ of each other; in $\%$ ) are shown for each location in blue, purple, green, and orange, respectively.

Northern Hemisphere mid-latitudes. Therefore, the choice of photolysis scheme is not the cause of the low bias in surface $\mathrm{O}_{3}$ in the Northern Hemisphere mid-latitudes. In relation to the comparison with the ACCENT ensemble, there was an improvement at $850 \mathrm{hPa}$ in the Northern Hemisphere tropics (RMSE reduced to $5.9 \mathrm{ppbv}$ ) but elsewhere, the model performance worsens with online photolysis. In the Northern Hemisphere extra-tropics, the RMSEs increased to 3.6 and $2.3 \mathrm{ppbv}$ at 500 and $750 \mathrm{hPa}$, respectively, but still remain smaller than the RMSEs associated with the ACCENT ensemble mean. Overall, online photolysis caused the RMSE of UKCA to increase from 7.7 to $9.9 \mathrm{pbbv}$, still within the 5.1$18.0 \mathrm{ppbv}$ range of RMSEs from all models in the ACCENT multi-model ensemble (Stevenson et al., 2006). The use of Fast-J also had a marked impact on global and zonal mean $\mathrm{OH}$. For example, Fast-J resulted in an increase in zonal annual mean $\mathrm{OH}$ of 3-170\%, depending on location. The smallest increase occurred at the surface in the tropics with the largest increase in regions where $\mathrm{OH}$ concentrations themselves are typically low (e.g. high latitude lower troposphere). Global mean $\mathrm{OH}$ increased by $40 \%$, resulting in a decrease in the $\mathrm{CH}_{4}$ lifetime from 9.96 to $7.12 \mathrm{yr}$, representing a decrease of over $28 \%$. Telford et al. (2013) also found that the $\mathrm{CH}_{4}$ lifetime decreased by $30 \%$, when switching from offline photolysis to Fast-JX in a HadGEM3-UKCA 


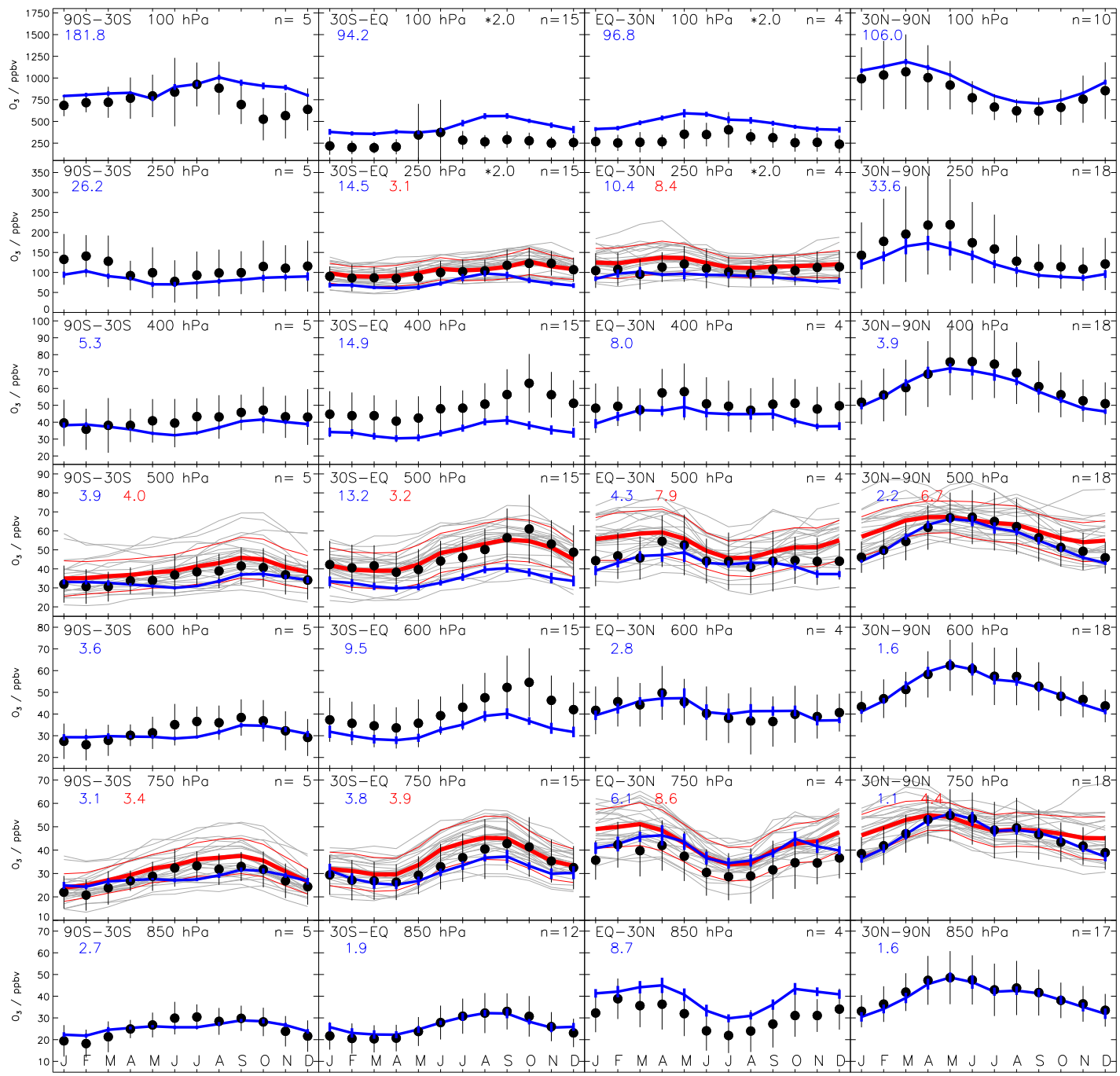

Fig. 26. Comparison of the annual cycle of $\mathrm{O}_{3}$ observations (black) with UKCA StdTrop (blue), the ACCENT ensemble of models (grey) and the ACCENT multi-model ensemble mean (red). Observations are taken from Logan et al. (1999) and Thompson et al. (2003a, b). The modelled and observed $\mathrm{O}_{3}$ concentrations at 100 and $250 \mathrm{hPa}$ in the latitude bands $30^{\circ} \mathrm{S}-\mathrm{EQ}$ and EQ-30 $\mathrm{N}$ have been multiplied by actor of 2. The root mean square error in ppbv between UKCA StdTrop and the observations is included in blue and the root mean square error in ppbv between the ACCENT multi-model mean and the observations is included in red.

coupled configuration and running with the TropIsop chemistry scheme.

The second sensitivity experiment (Expt. No. 2) carried out involved a switch in the model from free-running meteorology to a simulation in which the meteorology was nudged towards ERA-40 re-analysis data (Uppala et al., 2005) using the technique described in Telford et al. (2008). All other aspects of the model remained unchanged. In particular, the model was initialised using output for September from the control simulation. Then, using re-analysis data for 2005 and 2006, the model was allowed to spin up for 4 months, and run for the January-December 2006 period. The output was post-processed in order to be directly comparable with the TES observations by: (1) sampling the model at 3-hourly intervals, (2) interpolating the model output onto the 67 TES vertical pressure levels, and (3) applying the TES a priori profiles and averaging kernels in order to account for the measurement sensitivity at different times and locations. This post processing was identical to that described in Voulgarakis et al. (2011). Figure 28 shows a comparison between mid- and lower free tropospheric $\mathrm{CO}$ concentrations from TES and UKCA for the July-August 2006 period. Data from the 7 TES pressure levels between 800 and $400 \mathrm{hPa}$ were used. It can be seen that the HadGEM2-UKCA coupled model captures the spatial variability in observed concentrations fairly well. The Northern Hemisphere concentrations are higher than in the Southern Hemisphere and hot spots due to biomass burning are clearly evident in both the modelled 


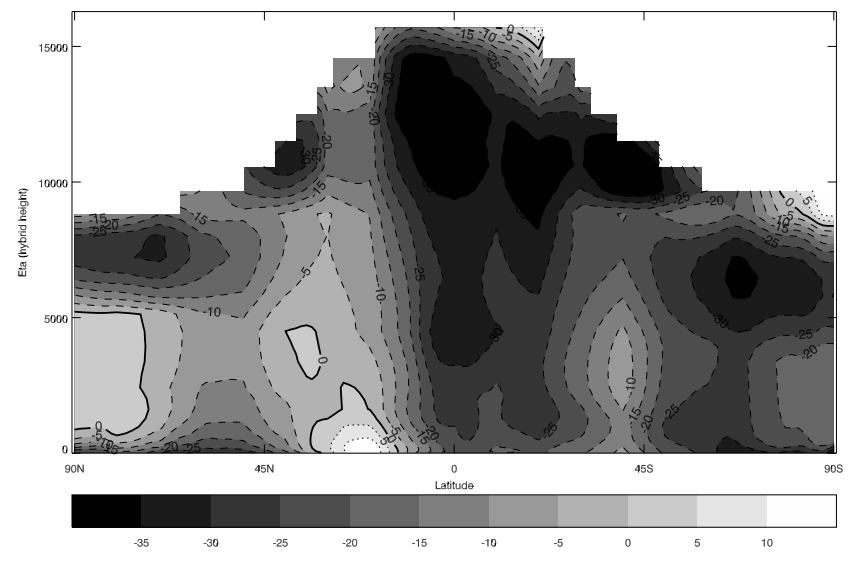

Fig. 27. Percentage difference in zonal annual mean $\mathrm{O}_{3}$ between UKCA and the Fortuin and Kelder (1998) climatology, i.e. 100 (model-climatology)/climatology. For the purposes of the comparison, the stratosphere (taken as $\mathrm{O}_{3}>150 \mathrm{ppbv}$ ) was masked.

and observed concentrations. Globally, the correlation coefficient between UKCA and TES CO observations is 0.90 and a least squares linear fit between UKCA and TES gives rise to a slope of 0.87 , indicating that modelled $\mathrm{CO}$ is biased low relative to the observations in the lower and mid-troposphere. For the December-January time period, the correlation coefficient and the slope of the least squares linear fit are 0.85 and 1.10, respectively. A comparison between modelled and observed $\mathrm{O}_{3}$ from TES suggests that the StdTrop chemistry scheme underestimates $\mathrm{O}_{3}$ in the middle and lower troposphere (the slopes of the least squares linear fits were 0.80 and 0.70 , for December-January and July-August, respectively). The corresponding correlation coefficients were 0.92 and 0.91 , respectively. The TES $\mathrm{O}_{3}$ observations themselves are biased high by 5-10\% (Nassar et al., 2008; Richards et al., 2008), which can partly explain some of the model's low bias with respect to TES. However, the lack of isoprene in StdTrop and its impact on chemical production of $\mathrm{O}_{3}$ is also a contributing factor (Wild, 2007). Further comparisons of UKCA relative to global $\mathrm{O}_{3}$ and $\mathrm{CO}$ observations from the TES instrument can be found in Voulgarakis et al. (2011), Young et al. (2013), and more recently, in Telford et al. (2013).

Modelled distributions of surface $\mathrm{O}_{3}$ in Sect. 5.5 suggested that concentrations in the northern mid-latitude continental regions are biased low. To investigate this further, a comparison between modelled and observed surface $\mathrm{O}_{3}$ at a greater number of surface sites was carried out. This confirmed that there was indeed a low bias at Hohenpeissenberg $\left(47.5^{\circ} \mathrm{N}, 11.0^{\circ} \mathrm{E}\right)$, for example, consistent with the comparison between UKCA and the Fortuin and Kelder climatology; the RMSE at Hohenpeissenberg at $900 \mathrm{hPa}$ was 8.4 ppbv. However, this low bias was not evident at all Northern Hemisphere locations (e.g. RSME at $900 \mathrm{hPa}$ at
Edmonton $\left(53.5^{\circ} \mathrm{N}, 113.3^{\circ} \mathrm{W}\right)$ was only $\left.2.5 \mathrm{ppbv}\right)$. Nevertheless, a number of sensitivity studies were carried out to investigate the potential cause of the low bias at Hohenpeissenberg. These are listed as Expts. 3-5 in Table 12. The choice of dry deposition scheme (Expt. No. 3) and the addition of surface emissions over the bottom 4 model levels (Expt. No. 4) had little impact on the comparison of surface $\mathrm{O}_{3}$ against the Fortuin and Kelder climatology or on the comparison with observations from (Logan et al., 1999) at Hohenpeissenberg. However, it was found that increasing the emissions of those NMVOCs considered in the StdTrop chemistry scheme (Expt. No. 5) resulted in improved comparisons at Hohenpeissenberg and Wallops Island, although it also resulted in overestimates at other Northern Hemisphere sites (e.g. Goose Bay, Churchill, and Ny Alesund). Nevertheless, it does demonstrate that the lack of treatment of NMVOCs in the StdTrop chemistry scheme has significant implications for surface and tropospheric $\mathrm{O}_{3}$ concentrations.

The final sensitivity experiment that was carried out (Expt. No. 6) involved a switch in the model from the StdTrop chemistry scheme to TropIsop. All other aspects of the model remained unchanged except that surface $\mathrm{CO}$ emissions were reduced by $354 \mathrm{TgCO} \mathrm{yr}^{-1}$, as outlined in Sect. 4.3, and monthly mean isoprene emissions totalling $503 \mathrm{Tg} \mathrm{C} \mathrm{yr}^{-1}$ (Guenther et al., 1995) were prescribed, on which a diurnal cycle was also imposed. The reduction in direct $\mathrm{CO}$ emissions and the inclusion of an isoprene degradation scheme had the effect of increasing $\mathrm{CO}$ concentrations in both hemispheres in the annual mean. Surface comparisons indicate that isoprene had the effect of reducing the relative annual mean bias and the root mean square error at Barrow, Mace Head, and Bermuda. However, in the Southern Hemisphere, the increase in $\mathrm{CO}$ concentration caused the performance of UKCA to be worse with TropIsop relative to StdTrop. For example, the relative annual mean bias and root mean square error change from $12.4 \%$ and $8.7 \mathrm{ppbv}$ with StdTrop to $35.6 \%$ and $16.6 \mathrm{ppbv}$ with TropIsop at Cape Grim $\left(41^{\circ} \mathrm{S}, 145^{\circ} \mathrm{E}\right)$. In relation to $\mathrm{NO}_{\mathrm{y}}$ partitioning, PAN concentrations compared with the Emmons et al. (2000) aircraft observations are much improved with TropIsop, and the underestimates in modelled PAN concentrations during SONEX and TRACE-A, in Fig. 21, are no longer evident. However, apart from Christmas Island, PAN is now overestimated in all other locations during PEM-Tropics B. The inclusion of the MIM also had the effect of increasing $\mathrm{O}_{3}$ between the surface and the mid-troposphere by $5-13 \mathrm{ppbv}$ in both hemispheres in the annual mean. This increase results in the RMSE at Hohenpeissenberg, for example, being reduced from 8.4 to $6.3 \mathrm{ppbv}$ but the comparison with the ozonesonde observations (Logan et al., 1999; Thompson et al., 2003a, b) used in the ACCENT comparison (Stevenson et al., 2006) indicates that the RMSEs between UKCA and the observations in all latitude bands at $850 \mathrm{hPa}$ are increased (e.g. RMSE in $30-90^{\circ} \mathrm{N}$ latitude band at $850 \mathrm{hPa}$ increased from 1.6 to $4.2 \mathrm{ppbv}$ ). This is compensated by improvements 

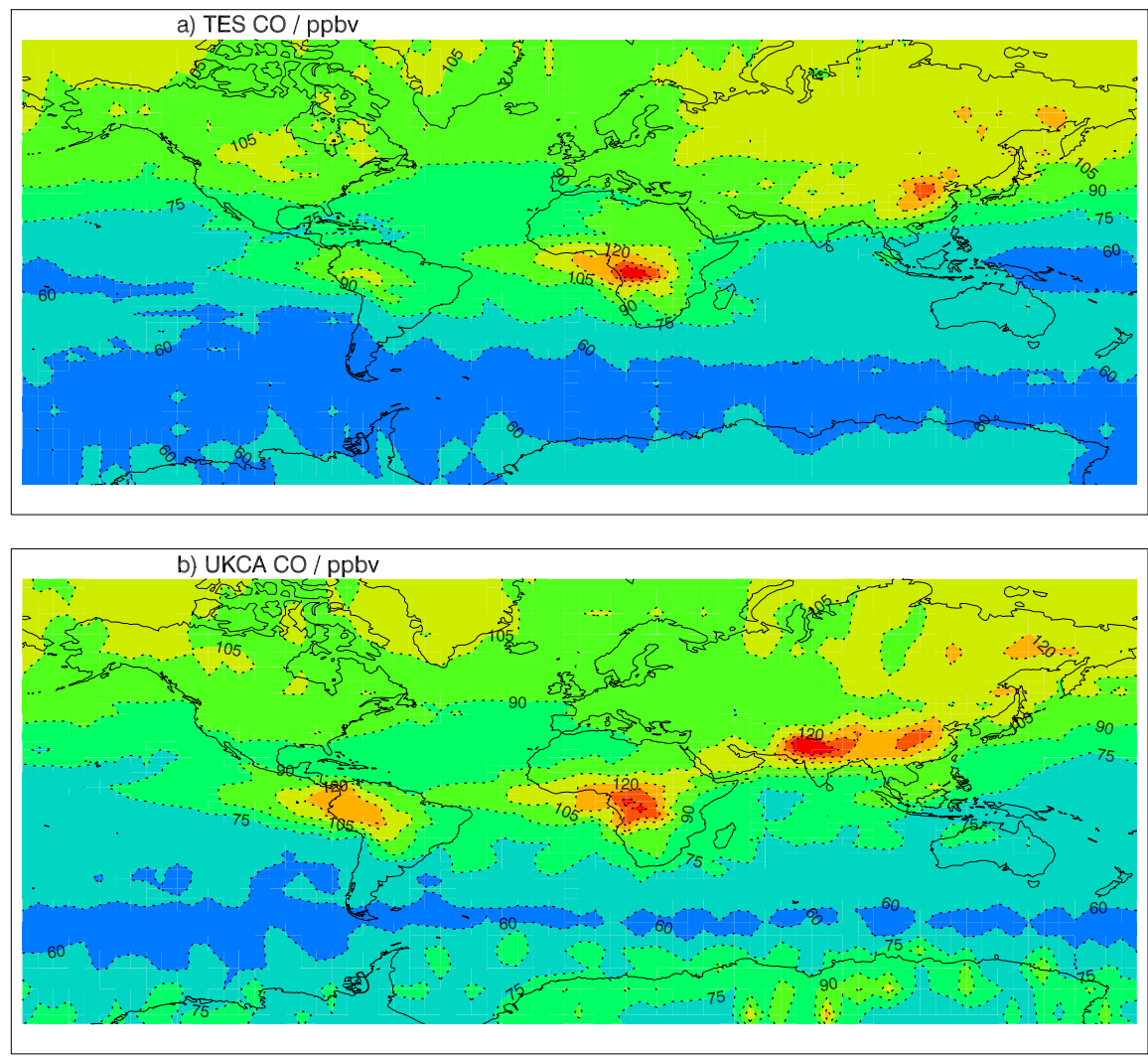

c) UKCA CO vs TES CO

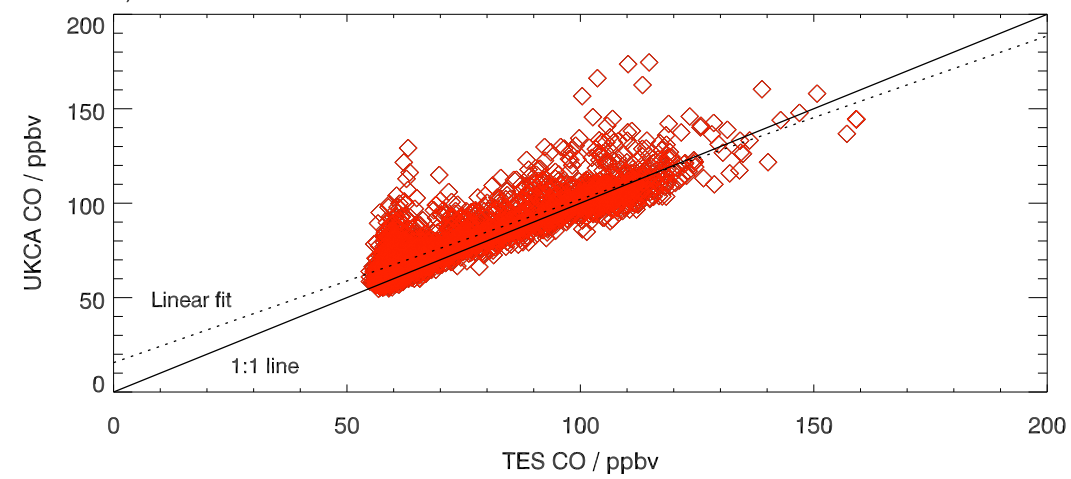

Fig. 28. Mid and lower free tropospheric CO concentrations from (a) TES and (b) UKCA StdTrop for July-August 2006. Data from 7 TES pressure levels between 800 and $400 \mathrm{hPa}$ were used. A scatter plot of UKCA CO concentrations against TES CO concentrations is shown in (c), along with the least squares linear fit and the 1:1 line. TES sampling and operators have been applied to the model output. The data have also been smoothed by averaging on a $4^{\circ} \times 5^{\circ}$ grid.

elsewhere (e.g. RMSE at $750 \mathrm{hPa}$ for $\mathrm{EQ}-30^{\circ} \mathrm{S}$ latitude band decreases from 3.8 to $2.8 \mathrm{ppbv}$ ). As a result, the overall RMSEs from UKCA StdTrop and UKCA TropIsop are very similar (7.7 and $7.8 \mathrm{ppbv}$, respectively). Figure 29 summarises the performance of UKCA TropIsop against the ACCENT multi-model ensemble and ozonesonde observations. In terms of global mean $\mathrm{OH}$, StdTrop had values of $0.8 \times 10^{6}$ molecules $\mathrm{cm}^{-3}$ and $1.03 \times 10^{6}{\text { molecules } \mathrm{cm}^{-3}}^{-3}$ based on weighting factors of air mass and reaction rate with $\mathrm{CH}_{4}$, respectively (Lawrence et al., 2001). The inclusion of the MIM changes these values to

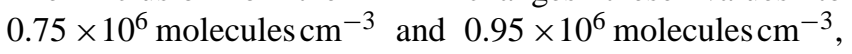
respectively, and is consistent with the air mass weighted value of $0.78 \times 10^{6}$ molecules $\mathrm{cm}^{-3}$ found in the HadGEM3UKCA TropIsop configuration (Telford et al., 2013). This decrease in global mean $\mathrm{OH}$ has the effect of increasing the lifetime of $\mathrm{CH}_{4}$ against $\mathrm{OH}$ from 9.96 to 10.86 yr. Further comparisons of the TropIsop chemistry scheme in the HadGEM3 


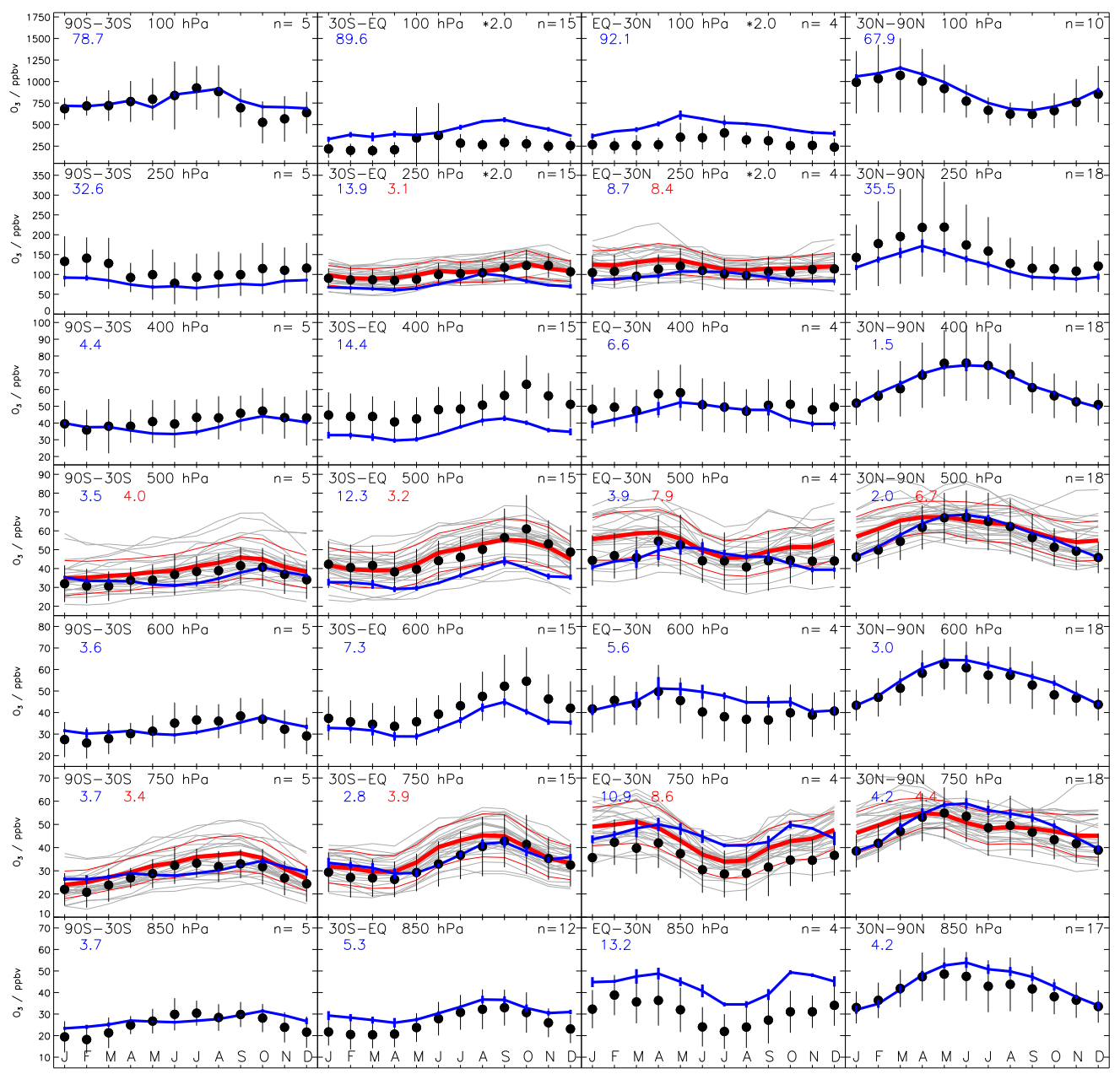

Fig. 29. Comparison of the annual cycle of $\mathrm{O}_{3}$ observations (black) with UKCA TropIsop (blue), the ACCENT ensemble of models (grey) and the ACCENT multi-model ensemble mean (red). Observations are taken from Logan et al. (1999) and Thompson et al. (2003a, b). The modelled and observed $\mathrm{O}_{3}$ concentrations at 100 and $250 \mathrm{hPa}$ in the latitude bands $30^{\circ} \mathrm{S}-\mathrm{EQ}$ and EQ- $30^{\circ} \mathrm{N}$ have been multiplied by a factor of 2. The root mean square error in ppbv between UKCA TropIsop and the observations is included in blue and the root mean square error in ppbv between the ACCENT multi-model mean and the observations is included in red.

climate model can also be found in Voulgarakis et al. (2011), and more recently, in Telford et al. (2013).

\section{Historical and future simulations}

One of the main objectives of StdTrop was the ability to represent $\mathrm{CH}_{4}$, tropospheric $\mathrm{O}_{3}$, and oxidant fields for sulphate aerosol formation in a climate model for use in centennial climate projections. Given the performance of the UKCA StdTrop chemistry scheme in an atmosphere-only configuration of HadGEM2 relative to present-day observations, the interactive chemistry scheme was included in all the centennial-scale simulations carried out with the Met Office Hadley Centre's climate model, HadGEM2-ES (Collins et al., 2011), for CMIP5 along with a few other climate modelling centres. For details on the historical and future $\mathrm{O}_{3}$-precursor emissions used in the CMIP5 simulations, the reader is referred to Lamarque et al. $(2010,2011)$; details on all the other HadGEM2-ES forcings can be found in Jones et al. (2011). Figure 30 shows a time series of the global mean surface $\mathrm{CH}_{4}$ and $\mathrm{O}_{3}$ concentrations, the tropospheric $\mathrm{CH}_{4}$ lifetime against $\mathrm{OH}$, and the tropospheric $\mathrm{O}_{3}$ burden for the historical period and out to 2100 for each of the representative concentration pathways (RCPs) RCP2.6 (van Vuuren et al., 2011), RCP4.5 (Thomson et al., 2011), RCP6.0 (Masui et al., 2011), and RCP8.5 (Riahi et al., 2011). In the historical and future runs, the global mean surface $\mathrm{CH}_{4}$ concentrations provided were used to prescribe the surface $\mathrm{CH}_{4}$ concentration in UKCA. The $\mathrm{CH}_{4}$ abundance aloft, however, evolved interactively along with the other gas-phase constituents. 

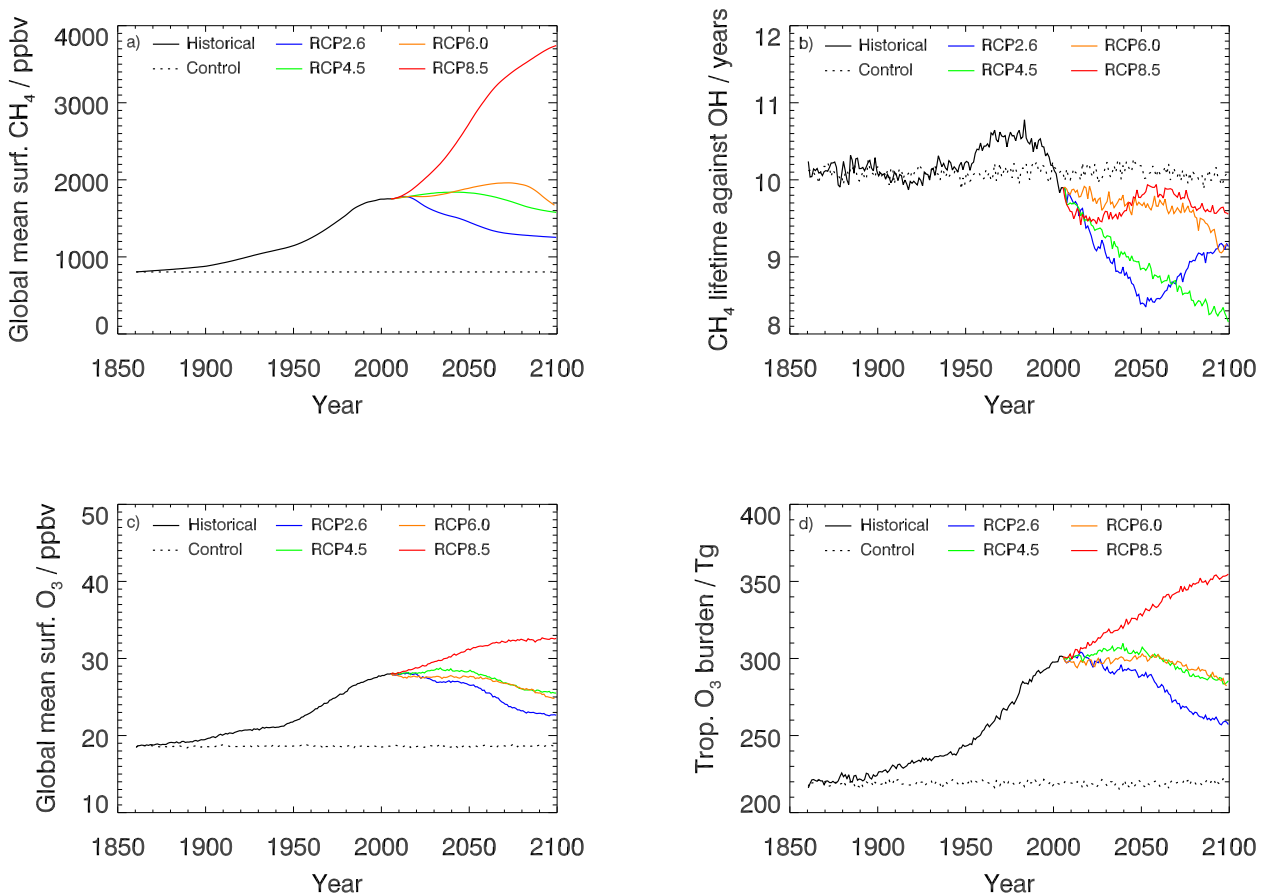

Fig. 30. Time series of (a) global mean surface $\mathrm{CH}_{4}$ concentration, (b) $\mathrm{CH}_{4}$ lifetime against $\mathrm{OH}$, (c) global mean surface $\mathrm{O}_{3}$ concentration, and (d) tropospheric $\mathrm{O}_{3}$ burden in the HadGEM2-ES simulations carried out for CMIP5. Simulations include a pre-industrial control, an historical simulation, and 4 future simulations using RCP2.6, RCP4.5, RCP6.0, and RCP8.5.

Figure 30b, in particular, shows how the tropospheric $\mathrm{CH}_{4}$ lifetime against $\mathrm{OH}$ varies over the historical and future periods relative to a pre-industrial control experiment. In the control, the $\mathrm{CH}_{4}$ lifetime is of the order of $10.1 \mathrm{yr}$. There is also some evidence of interannual and multidecadal variability in the lifetime. In the historical simulation, the $\mathrm{CH}_{4}$ lifetime remains similar to that in the pre-industrial control until about 1950. Thereafter, the lifetime increases, reaching a maximum value of $10.6 \mathrm{yr}$ before decreasing until the year 2000. This can be attributed to the SSTs in the coupled model being biased cold in the tropical oceans, thereby affecting atmospheric temperatures and humidity. In RCP2.6, the lifetime continues to decrease, reaching a minimum value of $8.4 \mathrm{yr}$ at 2050. Thereafter, the lifetime increases until 2100. Although Shindell et al. (2013) did not include a historical study, their time evolution of $\mathrm{CH}_{4}$ lifetime against $\mathrm{OH}$ from 2000-2100 is similar to that in this study. However, there are substantial differences in RCP8.5, for example. In the GISS model, Shindell et al. (2013) found that the lifetime was longer throughout the 21 st century than at the year 2000, whereas in HadGEM2 with UKCA StdTrop, $\mathrm{CH}_{4}$ lifetime reduces between 2000 and 2050, then increases before decreasing to a lifetime of $9.6 \mathrm{yr}$ at 2100 . Simulations of present-day and future $\mathrm{OH}$ and $\mathrm{CH}_{4}$ lifetime from the ACCMIP multimodel ensemble, and the implications of an offline photolysis scheme on modelled $\mathrm{CH}_{4}$ lifetime, are discussed further in Voulgarakis et al. (2013).
Tropospheric $\mathrm{O}_{3}$ increased during the historical simulation, giving rise to an increase in the global mean surface concentration of almost $9 \mathrm{ppbv}$ and in the tropospheric $\mathrm{O}_{3}$ burden of 7.3 Dobson units (DU), i.e. from $220 \pm 1.7 \mathrm{Tg} \mathrm{O} \mathrm{O}_{3}(20.5 \pm 0.16 \mathrm{DU})$ in the $1860 \mathrm{~s}$ to $300 \pm 1.6 \mathrm{Tg} \mathrm{O}_{3}(27.9 \pm 0.15 \mathrm{DU})$ for the $2000 \mathrm{~s}$. This increase in tropospheric $\mathrm{O}_{3}$ burden is similar in sign and magnitude to that from Cionni et al. (2011) and is at the lower end of the estimates from the ACCMIP models, i.e. $98 \pm 17 \mathrm{Tg} \mathrm{O}_{3}$ (Young et al., 2013). This can be most likely attributed to the lack of NMVOCs in StdTrop (Young et al., 2013). The time evolution of the increase, however, shows a slow increase from 1860 up to 1940, a more rapid increase between 1940 and 1980, followed by a slower increase between 1980 and the 2000s, reasonably consistent with that from Kawase et al. (2011). In the case of UKCA StdTrop, this increase in tropospheric $\mathrm{O}_{3}$ burden gives rise to a global mean radiative forcing of $0.24 \mathrm{~W} \mathrm{~m}^{-2}$ for the 2000s relative to the 1860 s based on a global mean normalised radiative forcing of $0.033 \mathrm{~W} \mathrm{~m}^{-2} \mathrm{DU}^{-1}$ by Gauss et al. (2006) and is consistent with that from Cionni et al. (2011). This estimate, however, does not take account that forcing by $\mathrm{O}_{3}$ varies strongly with region (Shindell et al., 2003) and it is lower than previous estimates (cf. $0.32 \pm 0.07 \mathrm{~W} \mathrm{~m}^{-2}$ from Gauss et al., 2006). Cionni et al. (2011) argue that it is primarily the result of 1850-2000 changes in biomass burning emissions being smaller than 
in the models used by Gauss et al. (2006). However, more recently, Stevenson et al. (2013) calculated radiative forcing from all the ACCMIP models using a number of different radiative transfer models and different tropopause definitions. They calculated an estimate of $0.3 \mathrm{~W} \mathrm{~m}^{-2}$ for UKCA StdTrop in HadGEM2, at the lower end of the ACCMIP ensemble mean $\left(0.36 \pm 0.06 \mathrm{~W} \mathrm{~m}^{-2}\right)$.

The simulations from year 2000 out to 2100 indicate that tropospheric $\mathrm{O}_{3}$ burden projections vary with RCP. In RCP2.6, the $\mathrm{O}_{3}$ burden continues to increase from 2000 until 2010, followed by a decrease until the end of the century. In RCP4.5, it reaches a maximum of $310 \mathrm{Tg} \mathrm{O}_{3}$ around 2040, and then declines to $285 \mathrm{Tg} \mathrm{O}_{3}$ at 2100. In RCP6.0, there is little change in the tropospheric $\mathrm{O}_{3}$ burden until around 2050-2055, it then starts to decline, reaching a value of $283 \mathrm{Tg} \mathrm{O}_{3}$ at 2100 . In contrast, RCP8.5 sees a continual increase in tropospheric $\mathrm{O}_{3}$ throughout the 21 st century, reaching a maximum of $355 \mathrm{Tg} \mathrm{O}_{3}$ at 2100 , with $\mathrm{CH}_{4}$ being the main driving factor. This time evolution is quite consistent with that from the CHASER CTM (Kawase et al., 2011), with reasonable agreement for the 2090s-2000s tropospheric $\mathrm{O}_{3}$ changes, i.e. -3.7 DU (RCP2.6), -1.2 DU (RCP4.5), -1.2 DU (RCP6.0), and +4.9 DU (RCP8.5). Cionni et al. (2011), likewise, calculated global annual mean tropospheric $\mathrm{O}_{3}$ column changes of $-5,-2,-2$, and +5 DU between the 2090s and 2000s for RCP2.6, RCP4.5, RCP6.0, and RCP8.5, respectively. The corresponding radiative forcing estimates from Stevenson et al. (2013) for StdTrop in HadGEM2 are $-0.15,-0.03$, and $+0.27 \mathrm{~W} \mathrm{~m}^{-2}$ for RCP2.6, RCP4.5, and RCP8.5, respectively. In particular, there is a clear consensus amongst the ACCMIP models on the sign and magnitude of the tropospheric $\mathrm{O}_{3}$ changes and the corresponding radiative forcing for each RCP.

\section{Conclusions}

This paper is Part 2 of a selection of papers, which aim to describe and evaluate the new UK Chemistry and Aerosol (UKCA) model. In particular, in this part, we evaluate aspects of dynamical processes which are important for tropospheric composition. A number of simple tracer experiments were set up, including ${ }^{222} \mathrm{Rn},{ }^{85} \mathrm{Kr}$, and ${ }^{210} \mathrm{~Pb}$, to assess boundary layer mixing, convective transport, inter-hemispheric exchange, and wet scavenging in the HadGEM2-UKCA coupled model. In relation to boundary layer mixing and convective transport, the performance of the HadGEM2-UKCA model was comparable to that of other models. It showed excellent comparison with surface observations at some sites but poorer performance at other sites. It reproduced the vertical profile of ${ }^{222} \mathrm{Rn}$ and ${ }^{210} \mathrm{~Pb}$ well, suggesting that boundary layer mixing and convective transport are well represented in the base dynamical model. The ${ }^{85} \mathrm{Kr}$ simulation indicated that the timescale for inter-hemispheric exchange was long relative to other model estimates and could have implications for meridional gradients of longlived tracers, such as $\mathrm{CH}_{4}$. Wet scavenging was also evaluated by means of a ${ }^{210} \mathrm{~Pb}$ tracer and results suggest that the HadGEM2-UKCA coupled model simulates the shape and absolute concentrations of observed profiles very well. Finally, global mass conservation was assessed and found to give a drift of less than $0.01 \%$ per year.

The climate model, HadGEM2, can be run with two tropospheric chemistry schemes. The first scheme, called StdTrop, is a fairly simple chemistry scheme but includes $\mathrm{O}_{\mathrm{x}}-\mathrm{NO}_{\mathrm{y}}-\mathrm{HO}_{\mathrm{x}}-\mathrm{CO}$ chemistry with near-explicit treatment of methane, ethane, propane, and acetone degradation (including formaldehyde, acetaldehyde, peroxy acetyl nitrate, and peroxy propionyl nitrate). The second chemistry scheme, called TropIsop, is an extension to StdTrop to include the species and reactions from the Mainz Isoprene Mechanism. Both chemistry schemes can be run with a choice of chemical solver (Backward Euler or Newton-Raphson), photolysis scheme (offline Hough scheme, Fast-J and Fast-JX) and dry deposition (prescribed deposition velocities or resistance approach), all of which are described. Descriptions of wet scavenging, upper boundary conditions, and emissions are also included.

The evaluation of the tropospheric configuration of UKCA focussed on the performance of StdTrop, particularly because the StdTrop chemistry scheme was included in all centennial model simulations with the HadGEM2-ES earth system model (Collins et al., 2011) by the Met Office Hadley Centre for the fifth Coupled Model Intercomparison Project (CMIP5). It was also included in the multi-model ensemble of the recent Atmospheric Chemistry and Climate Model Intercomparison Project (ACCMIP). In summary, it was found that the StdTrop chemistry scheme in HadGEM2 performs well in comparison with other models in simulating global and zonal mean distributions of $\mathrm{CH}_{4}, \mathrm{O}_{3}, \mathrm{CO}$, $\mathrm{NO}_{\mathrm{x}}$, and $\mathrm{OH}$. Surface comparisons of $\mathrm{CH}_{4}$ and $\mathrm{CO}$ were particularly good, but the model did less well in reproducing observed surface concentrations of $\mathrm{O}_{3}$. This can be attributed to the lack of non-methane hydrocarbon chemistry included in the chemical scheme, making it unsuitable for air quality purposes. However, from a climate forcing perspective, the UKCA StdTrop chemistry scheme coupled to the HadGEM2 climate model compares well with ozonesonde observations and other models in the mid- and upper troposphere. The global annual mean $\mathrm{OH}$ concentration is $0.8 \pm 0.03 \times 10^{6}$ molecules $\mathrm{cm}^{-3}$, resulting in a tropospheric $\mathrm{CH}_{4}$ lifetime against $\mathrm{OH}$ of $9.96 \mathrm{yr}$, in line with other published estimates.

Further developments of the UKCA model are already underway and/or completed. In particular, the aerosol component of the UKCA model, GLOMAP-mode, has been coupled to the UKCA chemistry schemes (with extensions for sulphur chemistry, for example) and will be described and evaluated in Part 3 of the UKCA paper series (Mann et al., 2014). In addition, an extended tropospheric chemistry 
scheme, ExtTC, has also been implemented in UKCA with enhanced coupling between the atmosphere and the land surface; this will be discussed in Part 4 of the series (Folberth et al., 2014). Furthermore, the TropIsop chemistry scheme has been combined with the stratospheric chemistry (Morgenstern et al., 2009); this combined chemistry scheme will be described in a forthcoming paper by Telford et al. (2014). As with other chemistry-aerosol models, UKCA is under continuous development; these developments will continue to be published in future papers.

Acknowledgements. The development of the UKCA model (www.ukca.ac.uk) and this work was supported by the Joint DECC/Defra Hadley Centre Climate Programme (GA01101) and the Natural Environment Research Council (NERC) through the NERC Centres for Atmospheric Science (NCAS) initiative. The authors would also like to thank all those who participated in the model intercomparison as part of European Union project Atmospheric Composition Change: the European Network of Excellence (ACCENT; http://www.accent-network.org), the results from which were used in Figs. 26 and 29.

Edited by: A. Stenke

\section{References}

Allen, D., Rood, R., Thompson, A., and Hudson, R.: Threedimensional radon 222 calculations using assimilated meteorological data and a convective mixing algorithm, J. Geophys. Res., 101, 6871-6881, 1996.

Andreae, M. and Merlet, P.: Emission of trace gases and aerosols from biomass burning, Global Biogeochem. Cy., 15, 955-966, 2001.

Archibald, A. T., Jenkin, M., and Shallcross, D.: An isoprene mechanism intercomparison, Atmos. Environ., 44, 5356-5364, 2010.

Archibald, A. T., Levine, J. G., Abraham, N. L., Cooke, M. C., Edwards, P. M., Heard, D. E., Jenkin, M. E., Karunaharan, A., Pike, R. C., Monks, P. S., Shallcross, D. E., Telford, P. J., Whalley, L. K., and Pyle, J. A.: Impacts of $\mathrm{HO}_{\mathrm{x}}$ regeneration and recycling in the oxidation of isoprene: consequences for the composition of past, present and future atmospheres, Geophys. Res. Lett., 38, L05804, doi:10.1029/2010GL046520, 2011.

Arneth, A., Monson, R. K., Schurgers, G., Niinemets, Ü., and Palmer, P. I.: Why are estimates of global terrestrial isoprene emissions so similar (and why is this not so for monoterpenes)?, Atmos. Chem. Phys., 8, 4605-4620, doi:10.5194/acp-8-46052008, 2008.

Ashmore, M.: Assessing the future global impacts of ozone on vegetation, Plant Cell Environ., 28, 949-964, 2005.

Atkinson, R., Baulch, D., Cox, R., Hampson, R., Kerr, J., Rossi, M., and Troe, J.: Evaluated kinetic and photochemical data for atmospheric chemistry: Supplement VIII, halogen species - IUPAC Subcommittee on Gas Kinetic Data Evaluation for Atmospheric Chemistry, J. Phys. Chem. Ref. Data, 29, 167-266, doi:10.1063/1.556058, 2000.

Atkinson, R., Baulch, D. L., Cox, R. A., Crowley, J. N., Hampson, R. F., Hynes, R. G., Jenkin, M. E., Rossi, M. J., and Troe, J.:
IUPAC Subcommittee for Gas Kinetic Data Evaluation, http:// www.iupac-kinetic.ch.cam.ac.uk/ (last access: November 2003), Summary for 2003, 2003.

Atkinson, R., Baulch, D. L., Cox, R. A., Crowley, J. N., Hampson, R. F., Hynes, R. G., Jenkin, M. E., Rossi, M. J., and Troe, J.: IUPAC Subcommittee for Gas Kinetic Data Evaluation, http: //www.iupac-kinetic.ch.cam.ac.uk/ (last access: October 2004), Summary for 2004, 2004.

Atkinson, R., Baulch, D. L., Cox, R. A., Crowley, J. N., Hampson, R. F., Hynes, R. G., Jenkin, M. E., Rossi, M. J., and Troe, J.: IUPAC Subcommittee for Gas Kinetic Data Evaluation, http://www.iupac-kinetic.ch.cam.ac.uk/ (last access: March 2005), Summary for 2005, 2005.

Balkanski, Y., Jacob, D., Gardner, G., Graustein, W., and Turekian, K.: Transport and residence times of tropospheric aerosols inferred from a global 3-dimensional simulation of Pb-210, J. Geophys. Res., 98, 20573-20586, doi:10.1029/93JD02456, 1993.

Barrie, L.: Background pollution in the arctic air-mass and its relevance to North-American acid-rain studies, Water Air Soil Poll., 30, 765-777, 1986.

Becker, K., Kleffmann, J., Kurtenbach, R., and Wiesen, P.: Solubility of nitrous acid (HONO) in sulfuric acid solutions, J. Phys. Chem., 100, 14984-14990, 1996.

Beer, R., Glavich, T. A., and Rider, D. M.: Tropospheric emission spectrometer for the Earth Observing System's Aura satellite, Appl. Optics, 40, 15, 2356-2367, doi:10.1364/AO.40.002356, 2001.

Bellouin, N., Rae, J., Jones, A., Johnson, C., Haywood, J., and Boucher, O.: Aerosol forcing in the Climate Model Intercomparison Project (CMIP5) simulations by HadGEM2-ES and the role of ammonium nitrate, J. Geophys. Res., 116, D20206, doi:10.1029/2011JD016074, 2011.

Bey, I., Jacob, D. J., Yantosca, R. M., Logan, J. A., Field, B. D., Fiore, A. M., Li, Q., Liu, H. Y., Mickley, L. J., and Schultz, M. G.: Global modeling of tropospheric chemistry with assimilated meteorology: Model description and evaluation, J. Geophys. Res., 106, 23073-23095, doi:10.1029/2001JD000807, 2001.

Bloss, C., Wagner, V., Jenkin, M. E., Volkamer, R., Bloss, W. J., Lee, J. D., Heard, D. E., Wirtz, K., Martin-Reviejo, M., Rea, G., Wenger, J. C., and Pilling, M. J.: Development of a detailed chemical mechanism (MCMv3.1) for the atmospheric oxidation of aromatic hydrocarbons, Atmos. Chem. Phys., 5, 641-664, doi:10.5194/acp-5-641-2005, 2005.

Broecker, W., Li, Y., and Cromwell, J.: Radium-226 and Radon222: concentrations in Atlantic and Pacific Oceans, Science, 158, 1307-1310, 1967.

Butchart, N., Scaife, A., Austin, J., Hare, S., and Knight, J.: Quasi-biennial oscillation in ozone in a coupled chemistry-climate model, J. Geophys. Res., 108, 4486, doi:10.1029/2002JD003004, D15, 2003.

Butler, T. M., Taraborrelli, D., Brühl, C., Fischer, H., Harder, H., Martinez, M., Williams, J., Lawrence, M. G., and Lelieveld, J.: Improved simulation of isoprene oxidation chemistry with the ECHAM5/MESSy chemistry-climate model: lessons from the GABRIEL airborne field campaign, Atmos. Chem. Phys., 8, 4529-4546, doi:10.5194/acp-8-4529-2008, 2008.

Carver, G., Brown, P., and Wild, O.: The ASAD atmospheric chemistry integration package and chemical reaction database, Comput. Phys. Commun., 105, 197-215, 1997. 
Chen, Y.-H. and Prinn, R.: Estimation of atmosphric methane emissions between 1996 and 2001 using a three-dimensional global chemical transport model, J. Geophys. Res., 111, D10307, doi:10.1029/2005JD006058, 2006.

Chevillard, A., Ciais, P., Karstens, U., Heimann, M., Schmidt, M., Levin, I., Jacob, D., Podzun, R., Kazan, V., Sartorius, H., and Weingartner, E.: Transport of ${ }^{222} \mathrm{Rn}$ using the regional model REMO: a detailed comparison with measurements over Europe, Tellus B, 54, 850-871, 2002.

Cionni, I., Eyring, V., Lamarque, J. F., Randel, W. J., Stevenson, D. S., Wu, F., Bodeker, G. E., Shepherd, T. G., Shindell, D. T., and Waugh, D. W.: Ozone database in support of CMIP5 simulations: results and corresponding radiative forcing, Atmos. Chem. Phys., 11, 11267-11292, doi:10.5194/acp11-11267-2011, 2011.

Collins, W., Stevenson, D., Johnson, C., and Derwent, R.: Tropospheric ozone in a global-scale three-dimensional Lagrangian model and its response to $\mathrm{NO}_{\mathrm{x}}$ emission controls, J. Atmos. Chem, 26, 223-274, 1997.

Collins, W., Derwent, R., Johnson, C., and Stevenson, D.: A comparison of two schemes for the convective transport of chemical species in a Lagrangian global chemistry model, Q. J. Roy. Meteor. Soc., 128, 991-1009, 2002.

Collins, W. J., Bellouin, N., Doutriaux-Boucher, M., Gedney, N., Halloran, P., Hinton, T., Hughes, J., Jones, C. D., Joshi, M., Liddicoat, S., Martin, G., O'Connor, F., Rae, J., Senior, C., Sitch, S., Totterdell, I., Wiltshire, A., and Woodward, S.: Development and evaluation of an Earth-System model - HadGEM2, Geosci. Model Dev., 4, 1051-1075, doi:10.5194/gmd-4-10512011, 2011.

Conen, F. and Robertson, L.: Latitudinal distribution of radon-222 flux from continents, Tellus, 54, 127-133, doi:10.1034/j.16000889.2002.00365.x, 2002.

Corbett, J. J. and Koehler, H. W.: Updated emissions from ocean shipping, J. Geophys. Res., 108, 4650, doi:10.1029/2003JD003751, D20, 2003.

CRC: CRC Handbook of Chemistry and Physics: A ReadyReference Book of Chemical and Physical Data, CRC Press, Boca Raton, FL, 84th Edn., 2003.

Davies, T., Cullen, M., Malcolm, A., Mawson, M., Staniforth, A., White, A., and Wood, N.: A new dynamical core for the Met Office's global and regional modelling of the atmosphere, Q. J. Roy. Meteor. Soc., 131, 1759-1782, 2005.

Denman, K., Brasseur, G., Chidthaisong, A., Ciais, P., Cox, P., Dickinson, R., Hauglustaine, D., Heinze, C., Holland, E., Jacob, D., Lohmann, U., Ramachandran, S., da Silva Dias, P., Wofsy, S., and Zhang, X.: Climate Change 2007: The Scientific Basis. Contribution of Working Group I to the Fourth Assessment Report of the Intergovernmental Panel on Climate Change, Chap. Couplings between changes in the climate system and biogeochemistry, Cambridge Univ. Press, Cambridge, 499-587, 2007.

Denning, A., Holzer, M., Gurney, K., Heimann, M., Law, R., Rayner, P., Fung, I., Fan, S.-M., Taguchi, S., Friedlingstein, P., Balkanski, Y., Taylor, J., Maiss, M., and Levin, I.: Threedimensional transport and concentration of $\mathrm{SF}_{6}$. A model intercomparison study (TransCom 2), Tellus B, 51, 266-297, 1999.

Dentener, F. and Crutzen, P.: Reaction of $\mathrm{N}_{2} \mathrm{O}_{5}$ on Tropospheric Aerosols: Impact on the Global Distributions of $\mathrm{NO}_{\mathrm{x}}, \mathrm{O}_{3}$, and OH, J. Geophys. Res., 98, 149-7163, 1993.
Dentener, F., Feichter, J., and Jeuken, A.: Simulation of the tranpsort of $\mathrm{Rn}^{222}$ using on-line and off-line global models at different horizontal resolutions: a detailed comparison with measurements, Tellus B, 51, 573-602, 1999.

Derwent, R. and Jenkin, M.: Hydrocarbons and the long-range transport of ozone and PAN across Europe, Atmos. Environ., 25, 1661-1678, 1991.

Dibb, J., Talbot, R., Klemm, K., Gregory, G., Singh, H., Bradshaw, J., and Sandholm, S.: Asian influence over the western North Pacific during the fall season: inferences from lead 210, soluble ionic species and ozone, J. Geophys. Res., 101, 1779-1792, 1996.

Dibb, J., Talbot, R., Lefer, B., Scheuer, E., Gregory, G., Browell, E., Bradshaw, J., Sandholm, S., and Singh, H.: Distributions of beryllium 7 and lead 210, and soluble aerosol-associated ionic species over the western Pacific: PEM West B, February-March 1994, J. Geophys. Res., 102, 28287-28302, 1997.

Dlugokencky, E., Houweling, S., Bruhwiler, L., Masarie, K., Lang, P., Miller, J., and Tans, P.: Atmospheric methane levels off: temporary pause or a new steady-state?, Geophys. Res. Lett., 30, 1992, doi:10.1029/2003GL018126, 2003.

Dlugokencky, E., Bruhwiler, L., White, J., Emmons, L., Novelli, P., Montzka, S., Masarie, K., Lang, P., Crotwell, A., Miller, J., and Gatti, L.: Observational constraints on recent increases in the atmospheric $\mathrm{CH}_{4}$ burden, Geophys. Res. Lett., 36, L18803, doi:10.1029/2009GL039780, 2009.

Emmerson, K. M. and Evans, M. J.: Comparison of tropospheric gas-phase chemistry schemes for use within global models, Atmos. Chem. Phys., 9, 1831-1845, doi:10.5194/acp-9-1831-2009, 2009.

Emmons, L., Hauglustaine, D., Muller, J., Carroll, M., Brasseur, G., Brunner, D., Staehelin, J., Thouret, V., and Marenco, A.: Data composites of airborne observations of tropospheric ozone and its precursors, J. Geophys. Res., 105, 20497-20538, 2000.

Endresen, Ø., Sørgård, E., Sundet, J., Dalsøren, S., Isaksen, I., Berglen, T., and Gravir, G.: Emission from international sea transportation and environmental impact, J. Geophys. Res., 108, 4560, doi:10.1029/2002JD002898, 2003.

Endresen, Ø., Sørgård, E., Behrens, H., Brett, P., and Isaksen, I.: A historical reconstruction of ships' fuel consumption and emissions, J. Geophys. Res., 112, D12301, doi:10.1029/2006JD007630, 2007.

Evans, M. and Jacob, D.: Impact of new laboratory studies of $\mathrm{N}_{2} \mathrm{O}_{5}$ hydrolysis on global model budgets of tropospheric nitrogen oxides, ozone, and OH, Geophys. Res. Lett., 32, L09813, doi:10.1029/2005GL022469, 2005.

Eyring, V., Kohler, H., van Aardenne, J., and Lauer, A.: Emissions from international shipping: 1. The last 50 years, J. Geophys. Res., 110, D17305, doi:10.1029/2004JD005619, 2005.

Eyring, V., Shepherd, T., and Waugh, D. E.: SPARC CCMVal: SPARC Report on the Evaluation of Chemistry-Climate Models, SPARC Report No. 5, WCRP-132, WMO/TD-No. 1526, http: //www.sparc-climate.org/ (last access: January 2014), 2010a.

Eyring, V., Isaksen, I. S. A., Berntsen, T., Collins, W. J., Corbett, J. J., Endresen, O., Grainger, R. G., Moldanova, J., Schlager, H., and Stevenson, D. S.: Transport impacts on atmosphere and climate: shipping, Atmos. Environ., 44, 4735-4771, doi:10.1016/j.atmosenv.2009.04.059, 2010 b. 
Fishman, J., Hoell, J., Bendura, R., McNeil, R., and Kirchhoff, V.: NASA GTE TRACE A experiment (September October 1992): Overview, J. Geophys. Res., 101, 23865-23879, doi:10.1029/96JD00123, 1996.

Folberth, G., Abraham, N., Dalvi, M., Johnson, C., Morgenstern, O., O'Connor, F. M., Pacifico, F., Young, P., Collins, W., and Pyle, J.: Evaluation of the new UKCA climate-composition model - Part 4: Extension to Tropospheric Chemistry and Biogeochemical Coupling between Atmosphere and Biosphere, Geosci. Model Dev. Discuss., in preparation, 2014.

Fortuin, J. and Kelder, H.: An ozone climatology based on ozonesonde and satellite measurements, J. Geophys. Res., 103, 31709-31734, 1998.

Fung, I., John, J., Lerner, J., Matthews, E., Prather, M., Steele, L., and Fraser, P.: 3-Dimensional model synthesis of the global methane cycle, J. Geophys. Res., 96, 13033-13065, 1991.

Ganzeveld, L. and Lelieveld, J.: Dry deposition parameterization in a chemistry general-circulation model and its influence on the distribution of reactive trace gases, J. Geophys. Res., 100, 20999-21012, doi:10.1029/95JD02266, 1995.

Gauss, M., Myhre, G., Isaksen, I. S. A., Grewe, V., Pitari, G., Wild, O., Collins, W. J., Dentener, F. J., Ellingsen, K., Gohar, L. K., Hauglustaine, D. A., Iachetti, D., Lamarque, F., Mancini, E., Mickley, L. J., Prather, M. J., Pyle, J. A., Sanderson, M. G., Shine, K. P., Stevenson, D. S., Sudo, K., Szopa, S., and Zeng, G.: Radiative forcing since preindustrial times due to ozone change in the troposphere and the lower stratosphere, Atmos. Chem. Phys., 6, 575-599, doi:10.5194/acp-6-575-2006, 2006.

Gent, P. R., Yeager, S. G., Neale, R. B., Levis, S., and Bailey, D. A.: Improvements in a half degree atmosphere/land version of the CCSM, Clim. Dynam., 34, 819-833, 2010.

Giannakopoulos, C.: Three dimensional modelling of the concentration and deposition of tropospheric trace gases, $\mathrm{Ph} . \mathrm{D}$. thesis, University of Cambridge, UK, 1998.

Giannakopoulos, C., Chipperfield, T., Law, K., and Pyle, J.: Validation and intercomparison of wet and dry deposition schemes using $\mathrm{Pb}-210$ in a global three-dimensional off-line chemical transport model, J. Geophys. Res., 104, 23761-23784, 1999.

GLOBALVIEW-CH 4 : Cooperative Atmospheric Data Integration Project - Methane, CD-ROM, NOAA ESRL, Boulder, Colorado, also available on Internet via anonymous FTP to ftp://ftp.cmdl. noaa.gov, Path: ccg/ch4/GLOBALVIEW, 2009.

Gordon, C., Cooper, C., Senior, C., Banks, H., Gregory, J., Johns, T., Mitchell, J., and Wood, R.: The simulation of SST, sea ice extents and ocean heat transports in a version of the Hadley Centre coupled model without flux adjustments, Clim. Dynam., 16, 147-168, 2000.

Gregory, D. and Rowntree, P.: A mass flux convection scheme with representation of cloud ensemble characteristics and stabilitydependent closure, Mon. Weather Rev., 118, 1483-1506, 1990.

Guelle, W., Balkanski, Y., Dibb, J., Schulz, M., and Dulac, F.: Wet deposition in a global size-dependent aerosol transport model 2. Influence of the scavenging scheme on $(210) \mathrm{Pb}$ vertical profiles, surface concentrations, and deposition, J. Geophys. Res., 103, 28875-28891, 1998a.

Guelle, W., Balkanski, Y., Schulz, M., Dulac, F., and Monfray, P.: Wet deposition in a global size-dependent aerosol transport model -1 . Comparison of a 1 year $\mathrm{Pb}-210$ simulation with ground measurements, Clim. Dynam., 103, 11429-11445, 1998b.

Guenther, A., Hewitt, C., Erickson, D., Fall, R., Geron, C., Graedel, T., Harley, P., Klinger, L., Lerdau, M., McKay, W., Pierce, T., Scholes, B., Steinbrecher, R., Tallamraju, R., Taylor, J., and Zimmerman, P.: A global-model of natural volatile organic-compound emissions, J. Geophys. Res., 100, 88738892, doi:10.1029/94JD02950, 1995.

Gupta, M., Douglass, A., Kawa, R., and Pawson, S.: Use of radon for evaluation of atmospheric transport models: sensitivity to emissions, Tellus B, 56, 404-412, doi:10.1111/j.16000889.2004.00124.x, 2004.

Hanson, D., Burkholder, J., Howard, C., and Ravishankara, A.: Measurement of oh and ho2 radical uptake coefficients on water and sulfuric-acid surfaces, J. Phys. Chem., 96, 4979-4985, 1992.

Hardiman, S. C., Butchart, N., Osprey, S. M., Gray, L. J., Bushell, A. C., and Hinton, T. J.: The Climatology of the Middle Atmosphere in a Vertically Extended Version of the Met Office's Climate Model. Part I: Mean State, J. Atmos. Sci., 67, 1509-1525, doi:10.1175/2009JAS3337.1, 2010.

Hartley, D. and Black, R.: Mechanistic analysis of interhemispheric transport, Geophys. Res. Lett., 22, 2945-2948, doi:10.1029/95GL02823, 1995.

Hauglustaine, D., Brasseur, G., Walters, S., Rasch, P., Muller, J., Emmons, L., and Carroll, C.: MOZART, a global chemical transport model for ozone and related chemical tracers 2. Model results and evaluation, J. Geophys. Res., 103, 28291-28335, 1998.

Hauglustaine, D., Hourdin, F., Jourdain, L., Filiberti, M., Walters, S., Lamarque, J., and Holland, E.: Interactive chemistry in the Laboratoire de Meteorologie Dynamique general circulation model: Description and background tropospheric chemistry evaluation, J. Geophys. Res., 109, D04314, doi:10.1029/2003JD003957, 2004.

Hayman, G., O’Connor, F. M., Dalvi, M., Clark, D., Huntingford, C., Gedney, N., Prigent, C., Buchwitz, M., Schneising, O., and Burrows, J.: First comparison of the HadGEM2 climate model with chemistry against Sciamachy atmospheric methane columns, Atmos. Chem. Phys. Discuss., in preparation, 2014.

Hertel, O., Berkowicz, R., Christensen, J., and Hov, O.: Test of 2 numerical schemes for use in atmospheric transport-chemistry models, Atmos. Environ., 27, 2591-2611, 1993.

Hewitt, H. T., Copsey, D., Culverwell, I. D., Harris, C. M., Hill, R. S. R., Keen, A. B., McLaren, A. J., and Hunke, E. C.: Design and implementation of the infrastructure of HadGEM3: the nextgeneration Met Office climate modelling system, Geosci. Model Dev., 4, 223-253, doi:10.5194/gmd-4-223-2011, 2011.

Hoell, J., Davis, D., Liu, S., Newell, R., Shipham, M., Akimoto, H., McNeal, R., Bendura, R., and Drewry, J.: Pacific exploratory Mission-West A (PEM-West A): September October 1991, J. Geophys. Res., 101, 1641-1653, doi:10.1029/95JD00622, 1996.

Hoerling, M., Schaack, T., and Lenzen, A.: A global analysis of stratospheric-tropospheric exchange during northern winter, Mon. Weather Rev., 121, 162-172, 1993.

Horowitz, L., Liang, J., Gardner, G., and Jacob, D.: Export of reactive nitrogen from North America during summertime: Sensitivity to hydrocarbon chemistry, J. Geophys. Res., 103, 1345113476, 1998. 
Hough, A.: The calculation of photolysis rates for use in global tropospheric modelling studies, Tech. Rep. 13259, At. Energy Res. Estab., Harwell, UK, 1988.

Houweling, S., Dentener, F., and Lelieveld, J.: The impact of nonmethane hydrocarbon compounds on tropospheric photochemistry, J. Geophys. Res., 103, 10673-10696, 1998.

Houweling, S., Kaminski, T., Dentener, F., Lelieveld, J., and Heimann, M.: Inverse modeling of methane sources and sinks using the adjoint of a global transport model, J. Geophys. Res., 104, 26137-26160, 1999.

Hoyle, C. R., Marécal, V., Russo, M. R., Allen, G., Arteta, J., Chemel, C., Chipperfield, M. P., D’Amato, F., Dessens, O., Feng, W., Hamilton, J. F., Harris, N. R. P., Hosking, J. S., Lewis, A. C., Morgenstern, O., Peter, T., Pyle, J. A., Reddmann, T., Richards, N. A. D., Telford, P. J., Tian, W., Viciani, S., Volz-Thomas, A., Wild, O., Yang, X., and Zeng, G.: Representation of tropical deep convection in atmospheric models Part 2: Tracer transport, Atmos. Chem. Phys., 11, 8103-8131, doi:10.5194/acp-11-8103-2011, 2011.

Jacob, D.: Heterogeneous chemistry and tropospheric ozone, Atmos. Environ., 34, 2131-2159, 2000.

Jacob, D., Prather, M., Wofsy, S., and McElroy, M.: Atmospheric distribution of $\mathrm{Kr}-85$ simulated with a general-circulation model, J. Geophys. Res., 92, 6614-6626, 1987.

Jacob, D., Prather, M., Rasch, P., Shia, R., Balkanski, Y., Beagley, S., Bergmann, D., Blackshear, W., Brown, M., Chiba, M., Chipperfield, M., deGrandpre, J., Dignon, J., Feichter, J., Genthon, C., Grose, W., Kasibhatla, P., Kohler, I., Kritz, M., Law, K., Penner, J., Ramonet, M., Reeves, C., Rotman, D., Stockwell, D., VanVelthoven, P., Verver, G., Wild, O., Yang, H., and Zimmermann, P.: Evaluation and intercomparison of global atmospheric transport models using Rn-222 and other short-lived tracers, J. Geophys. Res., 102, 5953-5970, 1997.

Jenkin, M., Saunders, S., Derwent, R., and Pilling, M.: Construction and application of a master chemical mechanism (MCM) for modelling tropospheric chemistry., Abstr. Pap. Am. Chem. S., 214, 116-COLL, 1997.

Jenkin, M. E., Saunders, S. M., Wagner, V., and Pilling, M. J.: Protocol for the development of the Master Chemical Mechanism, MCM v3 (Part B): tropospheric degradation of aromatic volatile organic compounds, Atmos. Chem. Phys., 3, 181-193, doi:10.5194/acp-3-181-2003, 2003.

Jöckel, P., Tost, H., Pozzer, A., Brühl, C., Buchholz, J., Ganzeveld, L., Hoor, P., Kerkweg, A., Lawrence, M. G., Sander, R., Steil, B., Stiller, G., Tanarhte, M., Taraborrelli, D., van Aardenne, J., and Lelieveld, J.: The atmospheric chemistry general circulation model ECHAM5/MESSy1: consistent simulation of ozone from the surface to the mesosphere, Atmos. Chem. Phys., 6, 5067-5104, doi:10.5194/acp-6-5067-2006, 2006.

Johns, T., Durman, C., Banks, H., Roberts, M., McLaren, A., Ridley, J., Senior, C., Williams, K., Jones, A., Rickard, G., Cusack, S., Ingram, W., Crucifix, M., Sexton, D., Joshi, M., Dong, B., Spencer, H., Hill, R., Gregory, J., Keen, A., Pardaens, A., Lowe, J., Bodas-Salcedo, A., Stark, S., and Searl, Y.: The new Hadley Centre Climate Model (HadGEM1): Evaluation of coupled simulations, J. Climate, 19, 1327-1353, doi:10.1175/JCLI3712.1, 2006.
Johnson, C., Collins, W., Stevenson, D., and Derwent, R.: Relative roles of climate and emissions changes on future tropospheric oxidant concentrations, J. Geophys. Res., 104, 18631-18645, 1999.

Johnson, C., Stevenson, D., Collins, W., and Derwent, R.: Role of climate feedback on methane and ozone studied with a coupled ocean-atmosphere-chemistry model., Geophys. Res. Lett., 28, 1723-1726, 2001.

Jones, C. D., Hughes, J. K., Bellouin, N., Hardiman, S. C., Jones, G. S., Knight, J., Liddicoat, S., O’Connor, F. M., Andres, R. J., Bell, C., Boo, K.-O., Bozzo, A., Butchart, N., Cadule, P., Corbin, K. D., Doutriaux-Boucher, M., Friedlingstein, P., Gornall, J., Gray, L., Halloran, P. R., Hurtt, G., Ingram, W. J., Lamarque, J.-F., Law, R. M., Meinshausen, M., Osprey, S., Palin, E. J., Parsons Chini, L., Raddatz, T., Sanderson, M. G., Sellar, A. A., Schurer, A., Valdes, P., Wood, N., Woodward, S., Yoshioka, M., and Zerroukat, M.: The HadGEM2-ES implementation of CMIP5 centennial simulations, Geosci. Model Dev., 4, 543-570, doi:10.5194/gmd-4-543-2011, 2011.

Josse, B., Simon, P., and Peuch, V.: Radon global simulations with the multiscale chemistry and transport model MOCAGE, Tellus B, 56, 339-356, 2004.

Kawase, H., Nagashima, T., Sudo, K., and Nozawa, T.: Future changes in tropospheric ozone under Representative Concentration Pathways (RCPs), Geophys. Res. Lett., 38, L05801, doi:10.1029/2010GL046402, 2011.

Kritz, M., Rosner, S., and Stockwell, D.: Validation of an offline three-dimensional chemical transport model using observed radon profiles - 1. Observations, J. Geophys. Res., 103, 84258432, 1998.

Lacis, A., Wuebbles, D., and Logan, J.: Radiative forcing of climate by changes in the vertical-distribution of ozone, J. Geophys. Res. 95, 9971-9981, 1990.

Lamarque, J.-F., Kinnison, D. E., Hess, P. G., and Vitt, F. M.: Simulated lower stratospheric trends between 1970 and 2005: Identifying the role of climate and composition changes, J. Geophys. Res., 113, D12301, doi:10.1029/2007JD009277, 2008.

Lamarque, J.-F., Bond, T. C., Eyring, V., Granier, C., Heil, A., Klimont, Z., Lee, D., Liousse, C., Mieville, A., Owen, B., Schultz, M. G., Shindell, D., Smith, S. J., Stehfest, E., Van Aardenne, J., Cooper, O. R., Kainuma, M., Mahowald, N., McConnell, J. R., Naik, V., Riahi, K., and van Vuuren, D. P.: Historical (1850-2000) gridded anthropogenic and biomass burning emissions of reactive gases and aerosols: methodology and application, Atmos. Chem. Phys., 10, 7017-7039, doi:10.5194/acp10-7017-2010, 2010.

Lamarque, J.-F., Kyle, G. P., Meinshausen, M., Riahi, K., Smith, S. J., van Vuuren, D. P., Conley, A. J., and Vitt, F.: Global and regional evolution of short-lived radiatively-active gases and aerosols in the Representative Concentration Pathways, Climatic Change, 109, 191-212, 2011.

Lamarque, J.-F., Shindell, D. T., Josse, B., Young, P. J., Cionni, I., Eyring, V., Bergmann, D., Cameron-Smith, P., Collins, W. J., Doherty, R., Dalsoren, S., Faluvegi, G., Folberth, G., Ghan, S. J., Horowitz, L. W., Lee, Y. H., MacKenzie, I. A., Nagashima, T., Naik, V., Plummer, D., Righi, M., Rumbold, S. T., Schulz, M., Skeie, R. B., Stevenson, D. S., Strode, S., Sudo, K., Szopa, S., Voulgarakis, A., and Zeng, G.: The Atmospheric Chemistry and Climate Model Intercomparison Project (ACCMIP): overview and description of models, simulations and climate diagnostics, 
Geosci. Model Dev., 6, 179-206, doi:10.5194/gmd-6-179-2013, 2013.

Lamb, B., Gay, D., Westberg, H., and Pierce, T.: A biogenic hydrocarbon emission inventory for the usa using a simple forest canopy model, Atmos. Environ., 27, 1673-1690, 1993.

Law, K. and Pyle, J.: Modeling trace gas budgets in the troposphere .1. ozone and odd nitrogen, J. Geophys. Res., 98, 18377-18400, 1993.

Law, K., Plantevin, P., Shallcross, D., Rogers, H., Pyle, J., Grouhel, C., Thouret, V., and Marenco, A.: Evaluation of modeled O3 using Measurement of Ozone by Airbus In-Service Aircraft (MOZAIC) data, J. Geophys. Res., 103, 25721-25737, doi:10.1029/98JD01482, 1998.

Law, R. and Corbin, K.: Simulating the atmospheric transport of $\mathrm{CO}_{2}$ and $\mathrm{SF}_{6}$ using the UK Met Office Unified Model, Tech. Rep. 2, Centre for Australian Westher and Climate Research (CAWCR), 2009.

Lawrence, M., Crutzen, P., Rasch, P., Eaton, B., and Mahowald, N.: A model for studies of tropospheric photochemistry: description, global distributions, and evaluation, J. Geophys. Res., 104, 26245-26277, doi:10.1029/1999JD900425, 1999.

Lawrence, M. G., Jöckel, P., and von Kuhlmann, R.: What does the global mean $\mathrm{OH}$ concentration tell us?, Atmos. Chem. Phys., 1, 37-49, doi:10.5194/acp-1-37-2001, 2001.

Lelieveld, J. and Crutzen, P.: The role of clouds in tropospheric photochemistry, J. Atmos. Chem., 12, 229-267, 1991.

Lelieveld, J., Butler, T. M., Crowley, J. N., Dillon, T. J., Fischer, H., Ganzeveld, L., Harder, H., Lawrence, M. G., Martinez, M., Taraborrelli, D., and Williams, J.: Atmospheric oxidation capacity sustained by a tropical forest, Nature, 452, 737-740, 2008.

Levin, I. and Hesshaimer, V.: Refining of atmospheric transport model entries by the globally observed passive tracer distributions of (85)krypton and sulfur hexafluoride (SF6), J. Geophys. Res., 101, 16745-16755, 1996.

Levy, H. I.: Normal atmosphere: Large radical and formaldehyde concentrations predicted, Science, 173, 141-143, 1971.

Lin, X., Zaucker, F., Hsie, E., Trainer, M., and McKeen, S.: Radon 222 simulations as a test of a three-dimensional regional transport model, J. Geophys. Res., 101, 29165-29177, 1996.

Lintner, B., Gilliland, A., and Fung, I.: Mechanisms of convectioninduced modulation of passive tracer interhemispheric transport interannual variability, J. Geophys. Res., 109, D13102, doi:10.1029/2003JD004306, 2004.

Lock, A., Brown, A., Bush, M., Martin, G., and Smith, R.: A new boundary layer mixing scheme. Part I: Scheme description and single-column model tests, Mon. Weather Rev., 128, 3187-3199, 2000.

Logan, J., Megretskaia, I., Miller, A., Tiao, G., Choi, D., Zhang, L., Stolarski, R., Labow, G., Hollandsworth, S., Bodeker, G., Claude, H., De Muer, D., Kerr, J., Tarasick, D., Oltmans, S., Johnson, B., Schmidlin, F., Staehelin, J., Viatte, P., and Uchino, O.: Trends in the vertical distribution of ozone: A comparison of two analyses of ozonesonde data, J. Geophys. Res., 104, 2637326399, doi:10.1029/1999JD900300, 1999.

Macintyre, H. L. and Evans, M. J.: Sensitivity of a global model to the uptake of $\mathrm{N}_{2} \mathrm{O}_{5}$ by tropospheric aerosol, Atmos. Chem. Phys., 10, 7409-7414, doi:10.5194/acp-10-7409-2010, 2010.

Macintyre, H. L. and Evans, M. J.: Parameterisation and impact of aerosol uptake of $\mathrm{HO}_{2}$ on a global tropospheric model,
Atmos. Chem. Phys., 11, 10965-10974, doi:10.5194/acp-1110965-2011, 2011.

Madronich, S., McKenzie, R., Bjorn, L., and Caldwell, M.: Changes in biologically active ultraviolet radiation reaching the Earth's surface, J. Photoch. Photobio. B, 46, 5-19, 1998.

Mann, G. W., Carslaw, K. S., Spracklen, D. V., Ridley, D. A., Manktelow, P. T., Chipperfield, M. P., Pickering, S. J., and Johnson, C. E.: Description and evaluation of GLOMAP-mode: a modal global aerosol microphysics model for the UKCA composition-climate model, Geosci. Model Dev., 3, 519-551, doi:10.5194/gmd-3-519-2010, 2010.

Mann, G., Johnson, C., Bellouin, N., Dalvi, M., Abraham, L., Carslaw, K. S., Boucher, O., Stier, P., Rae, J., Spracklen, D. V., Telford, P., Pyle, J., O’Connor, F. M., Carver, G., Pringle, K. J., and Woodhouse, M. T.: Evaluation of the new UKCA climatecomposition model - Part 3: Aerosols, Geosci. Model Develop. Discuss., in preparation, 2014.

Mao, J., Jacob, D. J., Evans, M. J., Olson, J. R., Ren, X., Brune, W. H., Clair, J. M. St., Crounse, J. D., Spencer, K. M., Beaver, M. R., Wennberg, P. O., Cubison, M. J., Jimenez, J. L., Fried, A., Weibring, P., Walega, J. G., Hall, S. R., Weinheimer, A. J., Cohen, R. C., Chen, G., Crawford, J. H., McNaughton, C., Clarke, A. D., Jaeglé, L., Fisher, J. A., Yantosca, R. M., Le Sager, P., and Carouge, C.: Chemistry of hydrogen oxide radicals $\left(\mathrm{HO}_{\mathrm{x}}\right)$ in the Arctic troposphere in spring, Atmos. Chem. Phys., 10, 5823-5838, doi:10.5194/acp-10-58232010, 2010.

Marenco, A., Jonquières, I., Gouget, H., and Nédélec, P.: Atmospheric Ozone as a Climate Gas, chap. Experimental determinations of meridional distribution and long term evolution of tropospheric ozone - Consequences on radiative forcing, SpringerVerlag, New York, USA, 305-319, 1995.

Martin, G., Ringer, M., Pope, V., Jones, A., Dearden, C., and Hinton, T.: The physical properties of the atmosphere in the new Hadley Centre Global Environmental Model (HadGEM1). Part I: Model description and global climatology, J. Climate, 19, 12741301, 2006.

Martin, G. M., Milton, S. F., Senior, C. A., Brooks, M. E., Ineson, S., Reichler, T., and Kim, J.: Analysis and reduction of systematic errors through a seamless approach to modeling weather and climate, J. Climate, 23, 5933-5957, 2010.

Master Chemical Mechanism v2: No longer available online, last access: February 2005, 2000.

Masui, T., Matsumoto, K., Hijioka, Y., Kinoshita, T., Nozawa, T., Ishiwatari, S., Kato, E., Shukla, P. R., Yamagata, Y., and Kainuma, M.: An emission pathway for stabilization at $6 \mathrm{~W} \mathrm{~m}^{-2}$ radiative forcing, Climatic Change, 109, 59-76, 2011.

Mickley, L. J., Jacob, D. J., and D. Rind, D.: Uncertainty in preindustrial abundance of tropospheric ozone: Implications for radiative forcing calculations, J. Geophys. Res., 106, 3389-3399, doi:10.1029/2000JD900594, 2001.

Morgenstern, O., Braesicke, P., Hurwitz, M. M., O'Connor, F. M., Bushell, A. C., Johnson, C. E., and Pyle, J. A.: The world avoided by the Montreal Protocol, Geophys. Res. Lett., 35, L16811, doi:10.1029/2008GL034590, 2008.

Morgenstern, O., Braesicke, P., O’Connor, F. M., Bushell, A. C., Johnson, C. E., Osprey, S. M., and Pyle, J. A.: Evaluation of the new UKCA climate-composition model - Part 1: The strato- 
sphere, Geosci. Model Dev., 2, 43-57, doi:10.5194/gmd-2-432009, 2009.

Müller, J. and Brasseur, G.: IMAGES - A 3-dimensional chemicaltransport model of the global troposphere, J. Geophys. Res., 100, 16445-16490, 1995.

Murphy, D. M. and Fahey, D. W.: An estimate of the flux of stratospheric reactive nitrogen and ozone into the troposphere, J. Geophys. Res., 99, 5325-5332, 1994.

Nassar, R., Logan, J. A., Worden, H. M., Megretskaia, I. A., Bowman, K. W., Osterman, G. B., Thompson, A. M., Tarasick, D. W., Austin, S., Claude, H., Dubey, M. K., Hocking, W. K., Johnson, B. J., Joseph, E., Merrill, J., Morris, G. A., Newchurch, M., Oltmans, S. J., Posny, F., Schmidlin, F. J., Vomel, H., Whiteman, D. N., and Witte, J. C.: Validation of Tropospheric Emission Spectrometer (TES) nadir ozone profiles using ozonesonde measurements, J. Geophys. Res., 113, D15S17, doi:10.1029/2007JD008819, 2008.

Neu, J. L., Prather, M. J., and Penner, J. E.: Global atmospheric chemistry: integrating over fractional cloud cover, J. Geophys. Res., 112, D11306, doi:10.1029/2006JD008007, 2007.

O'Connor, F. M., Johnson, C. E., Morgenstern, O., and Collins, W. J.: Interactions between tropospheric chemistry and climate model temperature and humidity biases, Geophys. Res. Lett., 36, L16801, doi:10.1029/2009GL039152, 2009.

O’Connor, F. M., Boucher, O., Gedney, N., Jones, C. D., Folberth, G. A., Coppell, R., Friedlingstein, P., Collins, W. J., Chappellaz, J., Ridley, J., and Johnson, C. E.: Possible role of wetlands, permafrost, and methane hydrates in the methane cycle under future climate change: a review, Rev. Geophys., 48, RG4005, doi:10.1029/2010RG000326, 2010.

Olsen, S. C., McLinden, C. A., and Prather, M. J.: The stratospheric $\mathrm{N}_{2} \mathrm{O}-\mathrm{NO}_{y}$ system: Testing uncertainties in a 3-D framework, J. Geophys. Res., 106, 28771-28784, 2001.

O'Sullivan, D., Lee, M., Noone, B., and Heikes, B.: Henry's law constant determinations for hydrogen peroxide, methyl hydroperoxide, hydroxymethyl hydroperoxide, ethyl hydroperoxide, and peroxyacetic acid, J. Phys. Chem., 100, 3241-3247, 1996.

Park, M., Randel, W., Kinnison, D., Garcia, R., and Choi, W.: Seasonal variation of methane, water vapor, and nitrogen oxides near the tropopause: satellite observations and model simulations, J. Geophys. Res., 109, D03302, doi:10.1029/2003JD003706, 2004.

Penner, J., Atherton, C., Dignon, J., Ghan, S., Walton, J., and Hameed, S.: Tropospheric nitrogen - a 3-dimensional study of sources, distributions, and deposition, J. Geophys. Res., 96, 959990, doi:10.1029/90JD02228, 1991.

Pfister, G. G., Emmons, L. K., Hess, P. G., Lamarque, J. F., Orlando, J. J., Walters, S., Guenther, A., Palmer, P. I., and Lawrence, P. J.: Contribution of isoprene to chemical budgets: a model tracer study with the NCAR CTM MOZART-4, J. Geophys. Res., 113, D05308, doi:10.1029/2007JD008948, 2008.

Pöschl, U., von Kuhlmann, R., Poisson, N., and Crutzen, P.: Development and intercomparison of condensed isoprene oxidation mechanisms for global atmospheric modeling, J. Atmos. Chem., 37, 29-52, doi:10.1023/A:1006391009798, 2000.

Prather, M., McElroy, M., Wofsy, S., Russell, G., and Rind, D.: Chemistry of the global troposphere - fluorocarbons as tracers of air motion, J. Geophys. Res., 92, 6579-6613, 1987.
Prather, M., Ehhalt, D., Dentener, F., Derwent, R., Dlugokencky, E., Holland, E., Isaksen, I., Katima, J., Kirchhoff, V., Matson, P., Midgley, P., and Wang, M.: Climate Change 2001: The Scientific Basis. Contribution of Working Group I to the Third Assessment Report of the Intergovernmental Panel on Climate Change, Chap. Atmospheric Chemistry and Greenhouse Gases, Cambridge Univ. Press, Cambridge, 239-287, 2001.

Preiss, N. and Genthon, C.: Use of a new database of lead 210 for global aerosol model validation, J. Geophys. Res., 102, 25347 25357, 1997.

Preiss, N., Melieres, M., and Pourchet, M.: A compilation of data on lead 210 concentration in surface air and fluxes at the air-surface and water-sediment interfaces, J. Geophys. Res., 101, 2884728862, 1996.

Price, C. and Rind, D.: A simple lightning parameterization for calculating global lightning distributions, J. Geophys. Res., 97, 9919-9933, 1992.

Price, C. and Rind, D.: What determines the cloud-to-ground lightning fraction in thunderstorms, Geophys. Res. Lett., 20, 463466, 1993.

Price, C. and Rind, D.: Modeling global lightning distributions in a general-circulation model, Mon. Weather Rev., 122, 1930-1939, 1994.

Price, C., Penner, J., and Prather, M.: $\mathrm{NO}_{\mathrm{x}}$ from lightning. 2. Constraints from the global atmospheric electric circuit, J. Geophys. Res., 102, 5943-5951, doi:10.1029/96JD02551, 1997.

Priestley, A.: A quasi-conservative version of the semi-Lagrangian advection scheme, Mon. Weather Rev., 121, 621-629, doi:10.1175/1520-0493(1993)121<0621:AQCVOT>2.0.CO;2, 1993.

Raper, J., Kleb, M., Jacob, D., Davis, D., Newell, R., Fuelberg, H., Bendura, R., Hoell, J., and McNeal, R.: Pacific Exploratory Mission in the tropical Pacific: PEM-Tropics B, March-April 1999, J. Geophys. Res., 106, 32401-32425, doi:10.1029/2000JD900833, 2001.

Rasch, P., Feichter, J., Law, K., Mahowald, N., Penner, J., Benkovitz, C., Genthon, C., Giannakopoulos, C., Kasibhatla, P., Koch, D., Levy, H., Maki, T., Prather, M., Roberts, D., Roelofs, G., Stevenson, D., Stockwell, Z., Taguchi, S., Kritz, M., Chipperfield, M., Baldocchi, D., McMurry, P., Barrie, L., Balkansi, Y., Chatfield, R., Kjellstrom, E., Lawrence, M., Lee, H., Lelieveld, J., Noone, K., Seinfeld, J., Stenchikov, G., Schwartz, S., Walcek, C., and Williamson, D.: A comparison of scavenging and deposition processes in global models: results from the WCRP Cambridge Workshop of 1995, Tellus B, 52, 1025-1056, 2000.

Ravishankara, A., Dunlea, E., Blitz, M., Dillon, T., Heard, D., Pilling, M., Strekowski, R., Nicovich, J., and Wine, P.: Redetermination of the rate coefficient for the reaction of $\mathrm{O}\left({ }^{1} \mathrm{D}\right)$ with $\mathrm{N}_{2}$, Geophys. Res. Lett., 29, 1745, doi:10.1029/2002GL014850, 2002.

Regimbal, J. and Mozurkewich, M.: Peroxynitric acid decay mechanisms and kinetics at low pH, J. Phys. Chem. A, 101, 8822-8829, 1997.

Riahi, K., Rao, S., Krey, V., Cho, C., Chirkov, V., Fischer, G., Kindermann, G., Nakicenovic, N., and Rafaj, P.: RCP 8.5-A scenario of comparatively high greenhouse gas emissions, Climatic Change, 109, 33-57, 2011.

Richards, N. A. D., Osterman, G.B., Browell, E.V., Hair, J. W., Avery, M., and Li, Q.: Validation of Tropospheric Emission Spec- 
trometer ozone profiles with aircraft observations during the intercontinental chemical transport experiment-B, J. Geophys. Res., 113, D16S29, doi:10.1029/2007JD008815, 2008.

Rind, D. and Lerner, J.: Use of on-line tracers as a diagnostic tool in general circulation model development. 1. Horizontal and vertical transport in the troposphere, J. Geophys. Res., 101, 1266712683, doi:10.1029/96JD00551, 1996.

Robertson, L., Stevenson, D., and Conen, F.: Test of a northwardsdecreasing Rn-222 source term by comparison of modelled and observed atmospheric Rn-222 concentrations, Tellus B, 57, 116123, 2005.

Roelofs, G. and Lelieveld, J.: Tropospheric ozone simulation with a chemistry-general circulation model: Influence of higher hydrocarbon chemistry, J. Geophys. Res., 105, 22697-22712, doi:10.1029/2000JD900316, 2000.

Russo, M. R., Marécal, V., Hoyle, C. R., Arteta, J., Chemel, C., Chipperfield, M. P., Dessens, O., Feng, W., Hosking, J. S., Telford, P. J., Wild, O., Yang, X., and Pyle, J. A.: Representation of tropical deep convection in atmospheric models - Part 1: Meteorology and comparison with satellite observations, Atmos. Chem. Phys., 11, 2765-2786, doi:10.5194/acp-11-27652011, 2011.

Sander, R. and Crutzen, P.: Model study indicating halogen activation and ozone destruction in polluted air masses transported to the sea, J. Geophys. Res., 101(D4), 9121-9138, doi:10.1029/95JD03793, 1996.

Sanderson, M., Jones, C., Collins, W., Johnson, C., and Derwent, R.: Effect of climate change on isoprene emissions and surface ozone levels, Geophys. Res. Lett., 30, doi:10.1029/2003GL017642, 2003.

Sanderson, M., Collins, W., Johnson, C., and Derwent, R.: Present and future acid deposition to ecosystems: the effect of climate change, Atmos. Environ., 40, 1275-1283, 2006.

Sanderson, M. G., Collins, W. J., Hemming, D. L., and Betts, R. A.: Stomatal conductance changes due to increasing carbon dioxide levels: projected impact on surface ozone levels, Tellus B, 59, 404-411, 2007.

Saunders, S., Jenkin, M., Derwent, R., and Pilling, M.: World Wide Web site of a Master Chemical Mechanism (MCM) for use in tropospheric chemistry models, Atmos. Environ., 31, 1249, doi:10.1016/S1352-2310(97)85197-7, 1997.

Saunders, S. M., Jenkin, M. E., Derwent, R. G., and Pilling, M. J.: Protocol for the development of the Master Chemical Mechanism, MCM v3 (Part A): tropospheric degradation of nonaromatic volatile organic compounds, Atmos. Chem. Phys., 3, 161-180, doi:10.5194/acp-3-161-2003, 2003.

Savage, N. H., Law, K. S., Pyle, J. A., Richter, A., Nüß, H., and Burrows, J. P.: Using GOME $\mathrm{NO}_{2}$ satellite data to examine regional differences in TOMCAT model performance, Atmos. Chem. Phys., 4, 1895-1912, doi:10.5194/acp-4-1895-2004, 2004.

Savage, N. H., Agnew, P., Davis, L. S., Ordóñez, C., Thorpe, R., Johnson, C. E., O’Connor, F. M., and Dalvi, M.: Air quality modelling using the Met Office Unified Model: model description and initial evaluation, Geosci. Model Dev., 6, 353-372, doi:10.5194/gmd-6-353-2013, 2013.

Seinfeld, J. and Pandis, S.: Atmospheric Chemistry and Physics: From Air Pollution to Climate Change, John Wiley \& Sons, Hoboken, New Jersey, USA, 2nd Edn., 2006.
Shindell, D. T., Faluvegi, G., and Bell, N.: Preindustrial-to-presentday radiative forcing by tropospheric ozone from improved simulations with the GISS chemistry-climate GCM, Atmos. Chem. Phys., 3, 1675-1702, doi:10.5194/acp-3-1675-2003, 2003.

Shindell, D. T., Faluvegi, G., Stevenson, D. S., Krol, M. C., Emmons, L. K., Lamarque, J.-F., Petron, G., Dentener, F. J., Ellingsen, K., Schultz, M. G., Wild, O., Amann, M., Atherton, C. S., Bergmann, D. J., Bey, I., Butler, T., Cofala, J., Collins, W. J., Derwent, R. G., Doherty, R. M., Drevet, J., Eskes, H. J., Fiore, A. M., Gauss, M., Hauglustaine, D. A., Horowitz, L. W., Isaksen, I. S. A., Lawrence, M. G., Montanaro, V., Muller, J. F., Pitari, G., Prather, M. J., Pyle, J. A., Rast, S., Rodriguez, J. M., Sanderson, M. G., Savage, N. H., Strahan, S. E., Sudo, K., Szopa, S., Unger, N., van Noije, T. P. C., and Zeng, G.: Multimodel simulations of carbon monoxide: Comparison with observations and projected near-future changes, J. Geophys. Res., 111, D19306, doi:10.1029/2006JD007100, 2006.

Shindell, D. T., Faluvegi, G., Koch, D. M., Schmidt, G. A., Unger, N., and Bauer, S. E.: Improved attribution of climate forcing to emissions, Science, 326, 716-718, doi:10.1126/science.1174760, 2009.

Shindell, D. T., Pechony, O., Voulgarakis, A., Faluvegi, G., Nazarenko, L., Lamarque, J.-F., Bowman, K., Milly, G., Kovari, B., Ruedy, R., and Schmidt, G.: Interactive ozone and methane chemistry in GISS-E2 historical and future climate simulations, Atmos. Chem. Phys., 13, 2653-2689, doi:10.5194/acp-13-26532013, 2013.

Sitch, S., Cox, P. M., Collins, W. J., and Huntingford, C.: Indirect radiative forcing of climate change through ozone effects on the land-carbon sink, Nature, 448, 791-794, doi:10.1038/nature06059, 2007.

Smith, R., Fowler, D., Sutton, M., Flechard, C., and Coyle, M.: Regional estimation of pollutant gas dry deposition in the UK: model description, sensitivity analyses and outputs, Atmos. Environ., 34, 3757-3777, 2000.

Sorteberg, A. and Hov, O.: Two parametrizations of the dry deposition exchange for $\mathrm{SO}_{2}$ and $\mathrm{NH}_{3}$ in a numerical model, Atmos. Environ., 30, 1823-1840, 1996.

Staudinger, J. and Roberts, P.: A critical compilation of Henry's law constant temperature dependence relations for organic compounds in dilute aqueous solutions, Chemosphere, 44, 561-576, 2001.

Stevenson, D., Collins, W., Johnson, C., and Derwent, R.: Intercomparison and evaluation of atmospheric transport in a Lagrangian model (STOCHEM), and an Eulerian model (UM), using Rn-222 as a short-lived tracer, Q. J. Roy. Meteor. Soc., 124, 2477-2491, 1998.

Stevenson, D., Dentener, F., Schultz, M., Ellingsen, K., van Noije, T., Wild, O., Zeng, G., Amann, M., Atherton, C., Bell, N., Bergmann, D., Bey, I., Butler, T., Cofala, J., Collins, W., Derwent, R., Doherty, R., Drevet, J., Eskes, H., Fiore, A., Gauss, M., Hauglustaine, D., Horowitz, L., Isaksen, I., Krol, M., Lamarque, J., Lawrence, M., Montanaro, V., Muller, J., Pitari, G., Prather, M., Pyle, J., Rast, S., Rodriguez, J., Sanderson, M., Savage, N., Shindell, D., Strahan, S., Sudo, K., and Szopa, S.: Multimodel ensemble simulations of present-day and nearfuture tropospheric ozone, J. Geophys. Res., 111, D08301, doi:10.1029/2005JD006338, 2006. 
Stevenson, D. S., Young, P. J., Naik, V., Lamarque, J.-F., Shindell, D. T., Voulgarakis, A., Skeie, R. B., Dalsoren, S. B., Myhre, G., Berntsen, T. K., Folberth, G. A., Rumbold, S. T., Collins, W. J., MacKenzie, I. A., Doherty, R. M., Zeng, G., van Noije, T. P. C., Strunk, A., Bergmann, D., Cameron-Smith, P., Plummer, D. A., Strode, S. A., Horowitz, L., Lee, Y. H., Szopa, S., Sudo, K., Nagashima, T., Josse, B., Cionni, I., Righi, M., Eyring, V., Conley, A., Bowman, K. W., and Wild, O.: Tropospheric ozone changes, radiative forcing and attribution to emissions in the Atmospheric Chemistry and Climate Model Inter-comparison Project (ACCMIP), Atmos. Chem. Phys., 13, 6, 3063-3085, doi:10.5194/acp-13-3063-2013, 2013.

Stockwell, D., Kritz, M., Chipperfield, M., and Pyle, J.: Validation of an off-line three-dimensional chemical transport model using observed radon profiles - 2. Model results, J. Geophys. Res., 103, 8433-8445, 1998.

Sudo, K., Takahashi, M., and Akimoto, H.: CHASER: a global chemical model of the troposphere - 2. Model results and evaluation, J. Geophys. Res., 107(D21), 4586, doi:10.1029/2001JD001114, 2002.

Telford, P. J., Braesicke, P., Morgenstern, O., and Pyle, J. A.: Technical Note: Description and assessment of a nudged version of the new dynamics Unified Model, Atmos. Chem. Phys., 8, 17011712, doi:10.5194/acp-8-1701-2008, 2008.

Telford, P. J., Lathière, J., Abraham, N. L., Archibald, A. T., Braesicke, P., Johnson, C. E., Morgenstern, O., O'Connor, F. M., Pike, R. C., Wild, O., Young, P. J., Beerling, D. J., Hewitt, C. N., and Pyle, J.: Effects of climate-induced changes in isoprene emissions after the eruption of Mount Pinatubo, Atmos. Chem. Phys., 10, 7117-7125, doi:10.5194/acp-10-7117-2010, 2010.

Telford, P. J., Abraham, N. L., Archibald, A. T., Braesicke, P., Dalvi, M., Morgenstern, O., O’Connor, F. M., Richards, N. A. D., and Pyle, J. A.: Implementation of the Fast-JX Photolysis scheme (v6.4) into the UKCA component of the MetUM chemistry-climate model (v7.3), Geosci. Model Dev., 6, 161177, doi:10.5194/gmd-6-161-2013, 2013.

Telford, P. J., Archibald, A. T., Abraham, N., Braesicke, P., Dalvi, M., Johnson, C., Keeble, J., O'Connor, F., Squire, O., and Pyle, J.: Evaluation of the UM-UKCA model configuration for Chemistry of the Stratosphere and Troposphere (CheST), Geosci. Model Dev. Discuss., in preparation, 2014.

The HadGEM2 Development Team: Martin, G. M., Bellouin, N., Collins, W. J., Culverwell, I. D., Halloran, P. R., Hardiman, S. C., Hinton, T. J., Jones, C. D., McDonald, R. E., McLaren, A. J., O'Connor, F. M., Roberts, M. J., Rodriguez, J. M., Woodward, S., Best, M. J., Brooks, M. E., Brown, A. R., Butchart, N., Dearden, C., Derbyshire, S. H., Dharssi, I., Doutriaux-Boucher, M., Edwards, J. M., Falloon, P. D., Gedney, N., Gray, L. J., Hewitt, H. T., Hobson, M., Huddleston, M. R., Hughes, J., Ineson, S., Ingram, W. J., James, P. M., Johns, T. C., Johnson, C. E., Jones, A., Jones, C. P., Joshi, M. M., Keen, A. B., Liddicoat, S., Lock, A. P., Maidens, A. V., Manners, J. C., Milton, S. F., Rae, J. G. L., Ridley, J. K., Sellar, A., Senior, C. A., Totterdell, I. J., Verhoef, A., Vidale, P. L., and Wiltshire, A.: The HadGEM2 family of Met Office Unified Model climate configurations, Geosci. Model Dev., 4, 723-757, doi:10.5194/gmd-4-723-2011, 2011.

Thompson, A., Singh, H., and Schlager, H.: Introduction to special section: Subsonic Assessment Ozone and Nitrogen Oxide Experiment (SONEX) and Pollution from Aircraft Emissions in the
North Atlantic Flight Corridor (POLINAT 2), J. Geophys. Res., 105, 3595-3603, doi:10.1029/2000JD900012, 2000.

Thompson, A., Witte, J., McPeters, R., Oltmans, S., Schmidlin, F., Logan, J., Fujiwara, M., Kirchhoff, V., Posny, F., Coetzee, G., Hoegger, B., Kawakami, S., Ogawa, T., Johnson, B., Vomel, H., and Labow, G.: Southern Hemisphere Additional Ozonesondes (SHADOZ) 1998-2000 tropical ozone climatology - 1 . Comparison with Total Ozone Mapping Spectrometer (TOMS) and ground-based measurements, J. Geophys. Res., 108, 8238, doi:10.1029/2001JD000967, D2, 2003a.

Thompson, A., Witte, J., Oltmans, S., Schmidlin, F., Logan, J., Fujiwara, M., Kirchhoff, V., Posny, F., Coetzee, G., Hoegger, B., Kawakami, S., Ogawa, T., Fortuin, J., and Kelder, H.: Southern Hemisphere Additional Ozonesondes (SHADOZ) 1998-2000 tropical ozone climatology - 2. Tropospheric variability and the zonal wave-one, J. Geophys. Res., 108, 8241, doi:10.1029/2002JD002241, D2, 2003b.

Thomson, A. M., Calvin, K. V., Smith, S. J., Kyle, G. P., Volke, A., Patel, P., Delgado-Arias, S., Bond-Lamberty, B., Wise, M. A., Clarke, L. E., and Edmonds, J. A.: RCP4.5: a pathway for stabilization of radiative forcing by 2100 , Climatic Change, 109, 77-94, 2011

Turekian, K., Nozaki, Y., and Benninger, L.: Geochemistry of atmospheric radon and radon products, Annu. Rev. Earth Pl. Sc., 5, 227-255, 1977.

Tyndall, G., Cox, R., Granier, C., Lesclaux, R., Moortgat, G., Pilling, M., Ravishankara, A., and Wallington, T.: Atmospheric chemistry of small organic peroxy radicals, J. Geophys. Res., 106, 12157-12182, doi:10.1029/2000JD900746, 2001.

Uppala, S. M., Kallberg, P. W., Simmons, A. J., Andrae, U., Bechtold, V. D., Fiorino, M., Gibson, J. K., Haseler, J., Hernandez, A., Kelly, G. A., Li, X., Onogi, K., Saarinen, S., Sokka, N., Allan, R. P., Andersson, E., Arpe, K., Balmaseda, M. A., Beljaars, A. C. M., Van De Berg, L., Bidlot, J., Bormann, N., Caires, S., Chevallier, F., Dethof, A., Dragosavac, M., Fisher, M., Fuentes, M., Hagemann, S., Holm, E., Hoskins, B. J., Isaksen, L., Janssen, P. A. E. M., Jenne, R., McNally, A. P., Mahfouf, J. F., Morcrette, J. J., Rayner, N. A., Saunders, R. W., Simon, P., Sterl, A., Trenberth, K. E., Untch, A., Vasiljevic, D., Viterbo, P., and Woollen, J.: The ERA-40 reanalysis, Q. J. Roy. Meteorol. Soc., 131, 29613012, 2005.

van Aardenne, J., Dentener, F., Olivier, J., and Peters, J.: The EDGAR 3.2 Fast Track 2000 dataset (32FT2000), available at: http://themasites.pbl.nl/tridion/en/themasites/edgar/ emission_data/edgar_32ft2000/index-2.html (last access: January 2014), 2005.

van der Werf, G. R., Randerson, J. T., Giglio, L., Collatz, G. J., Kasibhatla, P. S., and Arellano Jr., A. F.: Interannual variability in global biomass burning emissions from 1997 to 2004, Atmos. Chem. Phys., 6, 3423-3441, doi:10.5194/acp-6-3423-2006, 2006.

van Vuuren, D. P., Stehfest, E., den Elzen, M. G. J., Kram, T., van Vliet, J., Deetman, S., Isaac, M., Goldewijk, K. K., Hof, A., Beltran, A. M., Oostenrijk, R., and van Ruijven, B.: RCP2.6: exploring the possibility to keep global mean temperature increase below 2 degrees C, Climatic Change, 109, 95-116, 2011.

Vestreng, V., Rigler, E., Adams, M., Kindbom, K., Pacyna, J. M., van der Gon, H. D., Reis, S., Travnikovet, O.: EMEP: Inventory Review 2006: Emission data reported to the LRTAP Convention 
and NEC Directive, Stage 1, 2 and 3 review, Evaluation of inventories of HMs and POPs, EMEP MSC-W, Oslo, Norway, 2006.

von Kuhlmann, R., Lawrence, M., Crutzen, P., and Rasch, P.: A model for studies of tropospheric ozone and nonmethane hydrocarbons: Model description and ozone results, J. Geophys. Res., 108, 4729, doi:10.1029/2002JD003348, D23, 2003.

von Kuhlmann, R., Lawrence, M. G., Pöschl, U., and Crutzen, P. J.: Sensitivities in global scale modeling of isoprene, Atmos. Chem. Phys., 4, 1-17, doi:10.5194/acp-4-1-2004, 2004.

Voulgarakis, A., Wild, O., Savage, N. H., Carver, G. D., and Pyle, J. A.: Clouds, photolysis and regional tropospheric ozone budgets, Atmos. Chem. Phys., 9, 8235-8246, doi:10.5194/acp-9-82352009, 2009.

Voulgarakis, A., Savage, N. H., Wild, O., Braesicke, P., Young, P. J., Carver, G. D., and Pyle, J. A.: Interannual variability of tropospheric composition: the influence of changes in emissions, meteorology and clouds, Atmos. Chem. Phys., 10, 2491-2506, doi:10.5194/acp-10-2491-2010, 2010.

Voulgarakis, A., Telford, P. J., Aghedo, A. M., Braesicke, P., Faluvegi, G., Abraham, N. L., Bowman, K. W., Pyle, J. A., and Shindell, D. T.: Global multi-year $\mathrm{O}_{3}-\mathrm{CO}$ correlation patterns from models and TES satellite observations, Atmos. Chem. Phys., 11, 5819-5838, doi:10.5194/acp-11-5819-2011, 2011.

Voulgarakis, A., Naik, V., Lamarque, J.-F., Shindell, D. T., Young, P. J., Prather, M. J., Wild, O., Field, R. D., Bergmann, D., CameronSmith, P., Cionni, I., Collins, W. J., Dalsøren, S. B., Doherty, R. M., Eyring, V., Faluvegi, G., Folberth, G. A., Horowitz, L. W., Josse, B., McKenzie, I. A., Nagashima, T., Plummer, D. A., Righi, M., Rumbold, S. T., Stevenson, D. S., Strode, S. A., Sudo, K., Szopa, S., and Zeng, G.: Analysis of present day and future $\mathrm{OH}$ and methane lifetime in the ACCMIP simulations, Atmos. Chem. Phys., 13, 9, 4907-4916, doi:10.5194/acp-13-4907-2013, 2013.

Walton, J., MacCracken, M., and Ghan, S.: A global-scale lagrangian trace species model of transport, transformation, and removal processes, J. Geophys. Res., 93, 8339-8354, doi:10.1029/JD093iD07p08339, 1988.

Wang, C., Corbett, J., and Firestone, J.: Improving spatial representation of global ship emissions inventories, Environ. Sci. Tech., 42, 193-199, doi:10.1021/es0700799, 2008.

Wang, J., Logan, J., McElroy, M., Duncan, B., Megretskaia, I., and Yantosca, R.: A 3-D model analysis of the slowdown and interannual variability in the methane growth rate from 1988 to 1997 , Global Biogeochem. Cy., 18, doi:10.1029/2003GB002180, 2004.

Wang, K.-Y. and Shallcross, D. E.: A modelling study of tropospheric distributions of the trace gases $\mathrm{CFCl}_{3}$ and $\mathrm{CH}_{3} \mathrm{CCl}_{3}$ in the 1980s, Ann. Geophys., 18, 972-986, doi:10.1007/s00585000-0972-3, 2000.

Wang, Y., Jacob, D., and Logan, J.: Global simulation of tropospheric $\mathrm{O}-3-\mathrm{NO}_{\mathrm{X}}$-hydrocarbon chemistry 3. Origin of tropospheric ozone and effects of nonmethane hydrocarbons, J. Geophys. Res., 103, 10757-10767, doi:10.1029/98JD00156, 1998.

Waugh, D. W., Oman, L., Kawa, S. R., Stolarski, R. S., Pawson, S., Douglass, A. R., Newman, P. A., and Nielsen, J. E.: Impacts of climate change on stratospheric ozone recovery, Geophys. Res. Lett., 36, L03805, doi:10.1029/2008GL036223, 2009.

Weiss, W., Sittkus, A., Stockburger, H., Sartorius, H., and Munnich, K.: Large-scale atmospheric mixing derived from meridional profiles of KR-85, J. Geophys. Res., 88, 8574-8578, doi:10.1029/JC088iC13p08574, 1983.

Wesely, M.: Parameterization of surface resistances to gaseous dry deposition in regional-scale numerical-models, Atmos. Environ., 23, 1293-1304, 1989.

WHO: Health Aspects of Air Pollution with Particulate Matter, Ozone, and Nitrogen Dioxide, Report of a WHO Working Group EUR/03/5042688, World Health Organization European Centre for Environment and Health, Bonn, Germany, 2003.

Wild, O.: Modelling the global tropospheric ozone budget: exploring the variability in current models, Atmos. Chem. Phys., 7, 2643-2660, doi:10.5194/acp-7-2643-2007, 2007.

Wild, O. and Prather, M.: Excitation of the primary tropospheric chemical mode in a global three-dimensional model, J. Geophys. Res., 105, 24647-24660, doi:10.1029/2000JD900399, 2000.

Wild, O., Zhu, X., and Prather, M.: Fast-j: Accurate simulation of in- and below-cloud photolysis in tropospheric chemical models, J. Atmos. Chem., 37, 245-282, 2000.

WMO: Scientific Assessment of Ozone Depletion: 2010, World Meteorological Organization, Geneva, Switzerland, global Ozone Research and Monitoring Project - Report No. 52, 516 pp., 2011.

Yang, X., Cox, R., Warwick, N., Pyle, J., Carver, G., O’Connor, F. M., and Savage, N.: Tropospheric bromine chemistry and its impacts on ozone: a model study, J. Geophys. Res., 110, D23311, doi:10.1029/2005JD006244, 2005.

Yienger, J. and Levy, H.: Empirical-model of global soilbiogenic $\mathrm{NO}_{\mathrm{x}}$ emissions, J. Geophys. Res., 100, 11447-11464, doi:10.1029/95JD00370, 1995.

Young, P. J., Arneth, A., Schurgers, G., Zeng, G., and Pyle, J. A.: The $\mathrm{CO}_{2}$ inhibition of terrestrial isoprene emission significantly affects future ozone projections, Atmos. Chem. Phys., 9, 27932803, doi:10.5194/acp-9-2793-2009, 2009.

Young, P. J., Archibald, A. T., Bowman, K. W., Lamarque, J.-F., Naik, V., Stevenson, D. S., Tilmes, S., Voulgarakis, A., Wild, O., Bergmann, D., Cameron-Smith, P., Cionni, I., Collins, W. J., Dalsøren, S. B., Doherty, R. M., Eyring, V., Faluvegi, G., Horowitz, L. W., Josse, B., Lee, Y. H., MacKenzie, I. A., Nagashima, T., Plummer, D. A., Righi, M., Rumbold, S. T., Skeie, R. B., Shindell, D. T., Strode, S. A., Sudo, K., Szopa, S., and Zeng, G.: Preindustrial to end 21 st century projections of tropospheric ozone from the Atmospheric Chemistry and Climate Model Intercomparison Project (ACCMIP), Atmos. Chem. Phys., 13, 2063 2090, doi:10.5194/acp-13-2063-2013, 2013.

Zaucker, F., Daum, P., Wetterauer, U., Berkowitz, C., Kromer, B., and Broecker, W.: Atmospheric Rn-222 measurements during the 1993 NARE intensive, J. Geophys. Res., 101, 29149-29164, doi:10.1029/96JD02029, 1996.

Zeng, G. and Pyle, J.: Changes in tropospheric ozone between 2000 and 2100 modeled in a chemistry-climate model, Geophys. Res. Lett., 30, 1392, doi:10.1029/2002GL016708, 2003.

Zeng, G. and Pyle, J.: Influence of El Nino Southern Oscillation on stratosphere/troposphere exchange and the global tropospheric ozone budget, Geophys. Res. Lett., 32, L01814, doi:10.1029/2004GL021353, 2005.

Zeng, G., Pyle, J. A., and Young, P. J.: Impact of climate change on tropospheric ozone and its global budgets, Atmos. Chem. Phys., 8, 369-387, doi:10.5194/acp-8-369-2008, 2008. 